International Journal of Modern Physics D

(C) World Scientific Publishing Company

\title{
Building Models of Inflation in No-Scale Supergravity
}

\author{
John Ellis \\ Theoretical Particle Physics and Cosmology Group, Department of Physics, \\ King's College London, London WC2R 2LS, United Kingdom; \\ Theoretical Physics Department, CERN, CH-1211 Geneva 23, Switzerland; \\ National Institute of Chemical Physics and Biophysics, Rävala 10, 10143 Tallinn, Estonia \\ john.ellis@cern.ch \\ Marcos A. G. García \\ Instituto de Física Teórica (IFT) UAM-CSIC, Campus de Cantoblanco, 28049, Madrid, Spain \\ marcosa.garcia@uam.es \\ Natsumi Nagata \\ Department of Physics, University of Tokyo, Bunkyo-ku, Tokyo 113-0033, Japan \\ natsumi@hep-th.phys.s.u-tokyo.ac.jp \\ Dimitri V. Nanopoulos \\ George P. and Cynthia W. Mitchell Institute for Fundamental Physics and Astronomy, Texas \\ AEM University, College Station, TX 77843, USA; \\ Astroparticle Physics Group, Houston Advanced Research Center (HARC), \\ Mitchell Campus, Woodlands, TX 77381, USA; \\ Academy of Athens, Division of Natural Sciences, Athens 10679, Greece \\ dimitri@physics.tamu.edu \\ Keith A. Olive \\ William I. Fine Theoretical Physics Institute, School of Physics and Astronomy, \\ University of Minnesota, Minneapolis, MN 55455, USA \\ olive@umn.edu \\ Sarunas Verner \\ William I. Fine Theoretical Physics Institute, School of Physics and Astronomy, \\ University of Minnesota, Minneapolis, MN 55455, USA \\ nedzi002@umn.edu
}




\begin{abstract}
After reviewing the motivations for cosmological inflation formulated in the formalism of supersymmetry, we argue that the appropriate framework is that of no-scale supergravity. We then show how to construct within this framework inflationary models whose predictions for the tilt in the spectrum of scalar perturbations, $n_{s}$, and the ratio, $r$, of tensor and scalar perturbations coincide with those of the $R+R^{2}$ model of inflation proposed by Starobinsky. A more detailed study of no-scale supergravity reveals a structure that is closely related to that of $R^{2}$ modifications of the minimal Einstein-Hilbert action for general relativity, opening avenues for constructing no-scale de Sitter and anti-de Sitter models by combining pairs of Minkowski models, as well as generalizations of the original no-scale Starobinsky models of inflation. We then discuss the phenomenology of no-scale models of inflation, including inflaton decay and reheating, and then the construction of explicit scenarios based on $\mathrm{SU}(5), \mathrm{SO}(10)$ and string-motivated flipped $\mathrm{SU}(5) \times \mathrm{U}(1)$ GUT models. The latter provides a possible model of almost everything below the Planck scale, including neutrino masses and oscillations, the cosmological baryon asymmetry and cold dark matter, as well as $n_{s}$ and $r$.
\end{abstract}

KCL-PH-TH/2020-44, CERN-TH-2020-136, ACT-6-20, MI-TH-2024, UMN-TH-3926/20, FTPI-MINN-20/29, IFT-UAM/CSIC-20-126

Keywords: Supergravity; no-scale; inflation

\title{
1. Introduction
}

Inflation was initially proposed as a possible simultaneous solution to several fundamental problems in cosmology ${ }^{1}$ These included the horizon problem, i.e., the fact that the Universe is nearly homogeneous and isotropic on scales that are much larger than would ever have been within the range of causal contact in the conventional Big Bang cosmology framework, and the flatness problem, i.e., the fact the average density of the visible Universe today is very close to the critical density corresponding to a flat geometry with negligible curvature. Moreover, inflation also had the added bonus of suppressing by large factors the densities of unobserved massive relics from the first stages of the Big Bang, e.g., magnetic monopoles.

Most early formulations of inflationary model $\$^{2}$ were based on one or more effectively elementary scalar fields, though a proposal by Starobinsky ${ }^{3}$ was based on an extension of the Einstein-Hilbert action - which is linear in the Ricci tensor $R$ - to include an additional $R^{2}$ term. However, it was noticed later that this $R+R^{2}$ model is equivalent via a conformal transformation to the Einstein-Hilbert action complemented by a scalar field with a very specific form of effective potential. ${ }^{[4} \mathrm{In}$ this and many other scalar field models one finds that the change in the effective scalar inflaton field is $\mathcal{O}\left(M_{P}\right), \frac{5}{5}$ where $M_{P}=2.4 \times 10^{18} \mathrm{GeV}$ is the reduced Planck mass, suggesting that some assumption about gravity would be needed for their formulation.

A major step forward in the phenomenological interpretation of inflationary models came with the realization that the scalar inflaton field would be subject to quantum fluctuations 6.7 At the time, observational upper limits on possible perturbations in the cosmological microwave background (CMB) already imposed 
strong constraints on these fluctuations, which in turn implied that the effective inflaton potential must contain a small parameter, e.g., the inflaton mass and/or a potential coupling $\lambda$.

This realization posed a cosmological hierarchy problem: what was the dynamics permitting the inflaton mass to be $\ll M_{P}$ - a situation resembling that of the Higgs mass and the electroweak hierarchy problem - and/or the coupling $\lambda$ to be $\ll 1$. Three of us (JE, DN and KO) promptly suggested that this cosmological hierarchy problem of inflation cries out for stabilization by supersymmetry $[\sqrt[5]{8}[\sqrt[9]{9}$ Many supersymmetric models of inflation have been proposed subsequently.

The cosmological perturbations predicted by models of inflation share some generic features. The quantum fluctuations in the scalar inflaton field should be predominantly Gaussian in all models whose inflaton field rolls slowly down its effective potential, their spectrum would not in general be scale-invariant but exhibit a small tilt, $n_{s} \neq 1$, and the gravitational background would also be subject to small tensorial quantum fluctuations, with a ratio $r \ll 1$ relative to the larger scalar perturbations. Present measurements of the CMB confirm the presence of a tilt, 10 $n_{s}=0.965 \pm 0.004$, and set stringent upper limits on the tensor-to-scalar ratio, 11 $r<0.06$. These measurements conflict with the predictions of single-scalar inflationary models based on simple monomial potentials, but are completely consistent with the Starobinsky $R+R^{2}$ model, which predicts $r \simeq 0.003$.

The question then arises, how to write down supersymmetric models of inflation that are compatible with the available CMB data, with predictions similar to those of the Starobinsky model? More specifically, how may this be achieved within the framework of supergravity?

Everyone can agree that general relativity is an essential complication in cosmology, and we have already commented that the magnitude of the variation in the inflaton field confirms the need to include gravity in any complete model of inflation. Concretely, supergravity $[12] 13$ is the extension of supersymmetry to include gravity, so it is the appropriate framework for any cosmological scenario involving supersymmetry.

However, there is a significant theoretical hurdle to overcome ${ }^{14}$ Generic supergravity models with matter fields have effective potentials that do not obey the flatness conditions needed by slow-roll models of inflation, and have anti-de Sitter (AdS) vacua with field energies $-\mathcal{O}\left(m_{3 / 2}^{2} M_{P}^{2}\right)$, where $m_{3 / 2}$ is the gravitino mass. Moreover, a second desideratum for any particle physics model, and in particular any model of inflation, is that it not be incompatible with ultraviolet completion in some string model. So, are there any supergravity models that yield effective potentials with flat directions and no AdS 'holes', which emerge naturally as infrared limits of string models?

The affirmative answer to both questions is provided by no-scale supergravity models,$\frac{15}{18}$ so named because their effective potentials feature flat directions with no specific dynamical scale selected at the classical (tree) level, which have been derived from string models as their effective low-energy theories ${ }^{[19}$ 
In this paper we review the construction of models of inflation based on no-scale supergravity, paying particular attention to their predictions for inflationary observables and the extent to which they mimic those of the Starobinsky $R+R^{2}$ model. As we discuss, there is a natural close relation between the no-scale supergravity and Starobinsky models. ${ }^{20}$ We also discuss the evolution of the Universe after noscale supergravity inflation, paying particular attention to the reheating following inflaton decay and the production of gravitinos and supersymmetric dark matter. We discuss these issues, together with baryogenesis, neutrino masses and nucleon decay, in three grand unified theories (GUTs) incorporated into the no-scale supergravity framework, namely minimal $\mathrm{SU}(5), \mathrm{SO}(10)$ and flipped $\mathrm{SU}(5) \times \mathrm{U}(1)$. The latter is of particular interest, as $\mathrm{SU}(5) \times \mathrm{U}(1)$ is the only GUT gauge group whose symmetry can be broken down to the Standard Model (SM) via Higgs fields in the representations available in the weakly-coupled limits of heterotic string models. 21 The inflationary $\mathrm{SU}(5) \times \mathrm{U}(1)$ no-scale supergravity model is therefore a prototype theory of (almost) everything below the Planck scale.

The review is structured as follows. We begin in Section 2 with a brief motivation for supersymmetry, a review of the essential elements of supergravity with a focus on no-scale supergravity, as well as aspects of the Polonyi mode ${ }^{22}$ for supersymmetry breaking. Simple models of inflation in minimal and no-scale supergravity are discussed in Section 3 and the computation of inflationary observables are described in Section 4. A pioneering no-scale Starobinsky ${ }^{\sqrt{3}}$ inflation mode ${ }^{\sqrt{20}}$ is introduced in Section 5. At this point, we delve deeper into the structure of no-scale supergravity in Section 6, and the connections between no-scale supergravity and higher-order gravity theories are reviewed in Section 7. We discuss generalized no-scale models, Minkowski and de Sitter solutions in Section 8 and generalized inflationary models in Section 9. The stabilization of fields that do not drive inflation is discussed in Section 10. Phenomenological aspects of no-scale inflationary models including supersymmetry breaking and reheating are covered in Section 11. In Section 12, we review some UV completions of the inflationary models based on $\mathrm{SU}(5), \mathrm{SO}(10)$ and flipped $\mathrm{SU}(5) \times \mathrm{U}(1)$ grand unified theories. Our conclusions are summarized in Section 13.

\section{Supergravity Primer}

\subsection{Why Supersymmetry and Supergravity?}

Supersymmetry is thought to be an essential feature of string theory. However, the absence of supersymmetric particles at the $\mathrm{TeV}$ scale ${ }^{23}$ implies that it must be broken somewhere between there and the Planck scale. It should certainly be incorporated in any model of inflation if the supersymmetry-breaking scale is not larger than the scale of inflation, which is typically $\mathcal{O}\left(10^{13}\right) \mathrm{GeV}$. In fact, there are specific features of supersymmetric field theories that led some of us (JE, DN and $\mathrm{KO})^{\sqrt{8}}$ to propose that it should play an essential role in inflation, and therefore should be broken only at some scale $<\mathcal{O}\left(10^{13}\right) \mathrm{GeV}$. 
Supersymmetric field theories have unique renormalization properties: the masses of scalar particles have no quadratic divergences in any order of perturbation theory, and their trilinear and quartic couplings have only multiplicative logarithmic divergences. It was the absence of quadratic divergences that led to the suggestion that supersymmetry at the $\mathrm{TeV}$ scale could stabilize the electroweak scale and its hierarchy with the Planck scale, $\frac{24}{4}$ and the absence of non-multiplicative renormalization of trilinear and quartic couplings helped stabilize the hierarchy of mass scales in GUTs.

In view of the difficulties with the completion of the first-order phase transition in the first scalar field theory of inflation, 25 radiatively-driven GUT-based models of inflation were suggested ${ }^{26}$ However, in these models the magnitude of the effective potential was determined by the GUT gauge coupling, and was too large to be compatible with the CMB data. ${ }^{7}$ The solution proposed in Ref. 8 was to postulate that inflation was driven by a gauge singlet with a quartic scalar coupling that was kept naturally small by the fact that its renormalization was purely multiplicative in a supersymmetric theory. Moreover, the absence of quadratic divergences keeps the inflaton mass naturally small.

The field theories discussed in this early work featured global supersymmetry, but there were several reasons to extend them to theories with local supersymmetry, namely supergravity theories. ${ }^{27}$ One motivation was the principle that Nature abhors global symmetries, $\stackrel{28}{b}$ but embraces local symmetries such as the gauge symmetries of the Standard Model, and supergravity is the unique local (gauge) extension of global supersymmetry. Another motivation, already mentioned in the Introduction, is the fact that any treatment of cosmology should incorporate gravity, and supergravity is the unique framework combining it with supersymmetry. Finally, as a corollary of this observation, since the low-energy effective theory obtained from string theory necessarily contains gravity, and should also include supersymmetry so as to render inflation natural, as discussed above, it must be a supergravity theory.

\subsection{Structure of Supergravity Models}

We now review briefly the structure of the bosonic part of a generic supergravity Lagrangian to second order in the derivatives of the physical scalar fields, leaving until later aspects concerning auxiliary fields and fermions.

This supergravity bosonic Lagrangian for an $\mathcal{N}=1$ supersymmetric theory may be written in terms of a Hermitian function of complex chiral scalar fields $\phi^{i}$, called the Kähler potential, $K\left(\phi^{i}, \phi_{j}^{*}\right)$, that characterizes the geometry of the theory, and a holomorphic function of these fields, called the superpotential, $W\left(\phi^{i}\right)$, responsible for interactions among these fields and their fermionic partners. These may be combined into the function $G \equiv K+\ln |W|^{2}$. a The kinetic terms and scalar

${ }^{\mathrm{a}}$ We use natural units with $M_{P}=1$ in this Section. 
potential in the bosonic Lagrangian can then be written in the form

$$
\mathcal{L}=-\frac{1}{2} R+K_{i}^{j} \partial_{\mu} \phi^{i} \partial^{\mu} \phi_{j}^{*}-V-\frac{1}{4} \operatorname{Re}\left(f_{\alpha \beta}\right) F_{\mu \nu}^{\alpha} F^{\beta \mu \nu}-\frac{1}{4} \operatorname{Im}\left(f_{\alpha \beta}\right) F_{\mu \nu}^{\alpha} \tilde{F}^{\beta \mu \nu},
$$

where the first term is the minimal Einstein-Hilbert term of general relativity and in the second term $K_{i}^{j} \equiv \partial^{2} K / \partial \phi^{i} \partial \phi_{j}^{*}$. The effective scalar potential,

$$
V=e^{G}\left[G_{i}\left(G^{-1}\right)_{j}^{i} G^{j}-3\right],
$$

where $G_{i} \equiv \partial G / \partial \phi^{i}, G^{j} \equiv \partial G / \partial \phi_{j}^{*}$, and $\left(G^{-1}\right)_{j}^{i}$ is the inverse of the matrix of second derivatives of $G$. In addition, there are also $D$-term contributions for gauge non-singlet chiral fields. Finally, $f_{\alpha \beta}$ is the gauge kinetic function, which is in general a function of the chiral fields, $\phi^{i}$. b Minimal supergravity (mSUGRA) is characterized by a Kähler potential of the form

$$
K=\phi^{i} \phi_{i}^{*},
$$

in which case the effective potential (2) can be written in the form

$$
V\left(\phi^{i}, \phi_{j}^{*}\right)=e^{\phi^{i} \phi_{i}^{*}}\left[\left|W_{i}+\phi_{i}^{*} W\right|^{2}-3|W|^{2}\right],
$$

where $W_{i} \equiv \partial W / \partial \phi^{i}$.

The effective potential (4) illustrates some of the pitfalls of supergravity cosmology. The first is that, unlike the effective scalar potential in global supersymmetry, where $V=\left|W_{i}\right|^{2}$, the minimal supergravity potential is not positive semi-definitive. Indeed, the negative term $\propto|W|^{2}$ in (4) generates in general AdS 'holes' with depth $-\mathcal{O}\left(m_{3 / 2}^{2} M_{P}^{2}\right)$, inducing a cosmological instability. More generally, (4) does not have flat directions in field space except under special conditions such as those we discuss below. This is an issue for constructing models of inflation because, as we discuss in more detail below, a period of inflation that is long enough to solve the horizon and flatness problems should satisfy slow-roll conditions that require the scalar potential to have a(n almost) flat direction. This can be problematic in minimal supergravity (3), as the effective scalar potential (4) is proportional to $e^{K}$, which is $\left(1+\phi \phi^{*}+\ldots\right)$ for small field values. Scalars therefore typically pick up masses proportional to $V \sim H^{2}$, where $H$ is the Hubble parameter 14 29 violating the slow-roll conditions. This is known as the $\eta$-problem.

\subsection{No-Scale Supergravity}

These difficulties are avoided in no-scale supergravity models, of which the simplest is the single-field example with 15

$$
K=-3 \ln \left(T+T^{*}\right),
$$

${ }^{\mathrm{b}}$ For a review of local supersymmetry, see Ref. 13 
where $T$ may be identified with the volume modulus in a string compactification. This leads to an Einstein-Hilbert Lagrangian for gravity accompanied by the following kinetic term for the modulus field, derived from Eq. (1):

$$
\begin{aligned}
\mathcal{L}_{k i n} & =\frac{3}{\left(T+T^{*}\right)^{2}} \partial^{\mu} T \partial_{\mu} T^{*} \\
& =\frac{1}{12}\left(\partial_{\mu} K\right)^{2}+\frac{3}{4} e^{2 K / 3}\left|\partial_{\mu}\left(T-T^{*}\right)\right|^{2},
\end{aligned}
$$

where we note that, up to a factor of $\sqrt{6}, K$ has a canonical kinetic term. In the absence of a superpotential for the modulus, the effective scalar potential vanishes:

$$
V=0,
$$

which satisfies trivially the flatness condition, in particular the absence of negativeenergy AdS solutions.

In the minimal no-scale model (5) the single complex field $T$ parametrizes a non-compact $\mathrm{SU}(1,1) / \mathrm{U}(1)$ coset space. It can be generalized by including matter fields $\phi^{i}$ that parametrize, together with $T$, an $\mathrm{SU}(\mathrm{N}, 1) / \mathrm{SU}(\mathrm{N}) \times \mathrm{U}(1)$ coset space, defined by the Kähler potentialin ${ }^{1} \mathrm{C}$

$$
K=-3 \ln \left(T+T^{*}-\left|\phi_{i}\right|^{2} / 3\right) .
$$

In this case we find the following scalar-field Lagrangian

$$
\begin{aligned}
\mathcal{L}= & \frac{1}{12}\left(\partial_{\mu} K\right)^{2}+e^{K / 3}\left|\partial_{\mu} \phi^{i}\right|^{2} \\
& +\frac{3}{4} e^{2 K / 3}\left|\partial_{\mu}\left(T-T^{*}\right)+\frac{1}{3}\left(\phi_{i}^{*} \partial_{\mu} \phi^{i}-\phi^{i} \partial_{\mu} \phi_{i}^{*}\right)\right|^{2}-V,
\end{aligned}
$$

where the effective scalar potential can be written as

$$
V=e^{\frac{2}{3} K} \hat{V}=\frac{\hat{V}}{\left(\left(T+T^{*}\right)-\frac{1}{3}\left|\phi^{i}\right|^{2}\right)^{2}},
$$

with

$$
\hat{V} \equiv\left|W_{i}\right|^{2}+\frac{1}{3}\left(T+T^{*}\right)\left|W_{T}\right|^{2}+\frac{1}{3}\left(W_{T}\left(\phi_{i}^{*} W^{* i}-3 W^{*}\right)+\text { h.c. }\right) .
$$

We see that when $W_{T}=0$ the potential takes a form related to that in global supersymmetry, though with a proportionality factor of $e^{2 K / 3}$, as seen in Eq. 10, where $K$ is the canonically-redefined modulus. Hence large mass terms are not generated,$\stackrel{30}{ }$ and the $\eta$-problem is avoided $\stackrel{31}{.}$

$\overline{{ }^{c} \text { There are other generalizations based }}$ on other non-compact coset spaces, which also appear in some string models. 


\subsection{Supersymmetry Breaking}

In globally-supersymmetric models, supersymmetry is broken when $\langle F\rangle=W_{\phi} \neq 0$ for some field $\phi$. However, because the potential is simply equal to $\left|W_{\phi}\right|^{2}$, broken supersymmetry always leads to a non-zero vacuum energy density of the same order as the supersymmetry breaking. In contrast, in locally-supersymmetric models, supersymmetry is broken when an $F$-term given by

$$
F_{i}=-m_{3 / 2}\left(G^{-1}\right)_{i}^{j} G_{j}
$$

picks up a vacuum expectation value (VEV). In minimal $N=1$ supergravity, this corresponds to $\left\langle F_{i}\right\rangle=\left\langle e^{K / 2} D_{\phi^{i}} W\right\rangle=\left\langle e^{K / 2}\left(W_{i}+K_{i} W\right)\right\rangle \neq 0$. In this case, because of the form of the potential in Eq. (4), it is possible to break supersymmetry and at the same time cancel the vacuum energy density, with a gravitino mass given by $m_{3 / 2}=e^{\langle G\rangle / 2}$.

The simplest example of a model that breaks supersymmetry and allows $V=0$ is the Polonyi model $[22$ The model is based on adding a single chiral superfield that breaks supersymmetry spontaneously through the super-Higgs mechanism, $, 12,32$ which has two physical scalar fields whose fermionic partners are eaten by the gravitino. In the simplest version of the model, the superpotential is separable in the Polonyi field, $z$, and the matter fields, $\phi^{i}$ :

$$
W\left(z, \phi^{i}\right)=f(z)+g\left(\phi^{i}\right),
$$

with the particular choice

$$
f(z)=\mu(z+\zeta)
$$

where $\zeta$ is a constant. Ignoring for the moment the matter fields, the potential for $z$ is

$$
V\left(z, z^{*}\right)=e^{z z^{*}} \mu^{2}\left[\left|1+z^{*}(z+\zeta)\right|^{2}-3|(z+\zeta)|^{2}\right]
$$

where we have assumed a minimal supergravity framework, i.e., $K=z z^{*}$. Minimizing the potential and insisting that $V(\langle z\rangle)=0$ (which requires fine-tuning), we find $\langle z\rangle=\sqrt{3}-1$ and $\zeta=2-\sqrt{3}$. The Polonyi potential is shown in Fig. 1

In this example, the masses of the two real scalars, denoted by $A$ and $B$, are

$$
m_{A}^{2}=2 \sqrt{3} m_{3 / 2}^{2} \quad m_{B}^{2}=2(2-\sqrt{3}) m_{3 / 2}^{2},
$$

where the gravitino mass

$$
m_{3 / 2}=e^{\langle G\rangle / 2}=e^{2-\sqrt{3}} \mu .
$$

These satisfy the mass relation $m_{A}^{2}+m_{B}^{2}=4 m_{3 / 2}^{2}$, which is a consequence of the supertrace formula in supergravity 12

Including now the matter fields, one can calculate their soft supersymmetrybreaking mass terms ${ }^{33}$ by evaluating the potential at $\langle z\rangle$ and dropping terms in the 


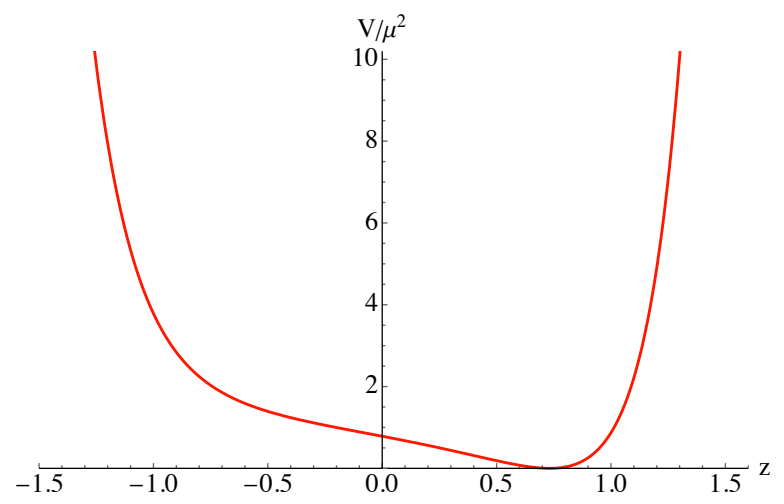

Fig. 1. The effective potential in the simplest Polonyi model of supersymmetry breaking in supergravity.

potential that are of dimension higher than four, as these would scale with inverse powers of the Planck mass. The scalar potential then becomes

$$
V=m_{3 / 2} e^{(2-\sqrt{3})}\left(\phi^{i} \frac{\partial g}{\partial \phi^{i}}-\sqrt{3} g+\text { h.c. }\right)+m_{3 / 2}^{2} \phi^{i} \phi_{i}^{*} .
$$

Rescaling the superpotential by a factor $e^{\sqrt{3}-2}$, and noting that $\sum \phi \partial g / \partial \phi=3 g$ for trilinear terms and $\sum \phi \partial g / \partial \phi=2 g$ for bilinear terms, we can read off the soft masses

$$
m_{0}=m_{3 / 2}, \quad B_{0}=(2-\sqrt{3}) m_{3 / 2}, \quad A_{0}=(3-\sqrt{3}) m_{3 / 2},
$$

where $m_{0}$ is a universal soft scalar mass, $A_{0}$ is universal soft trilinear term, and $B_{0}$ is a universal soft bilinear term.

This simple paradigm for supersymmetry breaking has important consequences for the minimal supersymmetric extension of the Standard Model (MSSM). The soft masses in Eq. 19) represent universal boundary conditions for all scalar masses, $A$-terms and $B$-terms. In the constrained MSSM (CMSSM), 34 all scalars are assumed to be universal at some high energy scale often taken to be the GUT scale, $A$-terms are left free but universal, and the $B$-term (there is only one in the MSSM) is obtained from the minimization of the Higgs potential when $\tan \beta$ (the ratio of the two Higgs VEVs) is taken as a free parameter.

A supersymmetry-breaking gaugino mass requires a non-trivial gauge kinetic function for a canonically-normalized gauge field,

$$
m_{1 / 2}=\left|\frac{1}{2} e^{G / 2} \frac{f_{i}^{*}}{\operatorname{Re} f}\left(G^{-1}\right)_{j}^{i} G^{j}\right|,
$$

where we have assumed a universal gauge kinetic function, $f_{\alpha \beta}=f \delta_{\alpha \beta}$.

In addition to the gaugino mass, $m_{1 / 2}, m_{0}, A_{0}$, and $\tan \beta$ make up the four continuous free input parameters of the CMSSM. The boundary conditions in Eq. (19) are more restrictive, as $m_{0}, A_{0}$, and $B_{0}$ are all determined by the gravitino mass. 
Indeed, the relation $B_{0}=A_{0}-m_{0}$, is a common feature of many models based on supergravity 40

However, this simple paradigm for supersymmetry breaking is not without problems. In particular, the potential shown in Fig. 1 has a serious cosmological problem $\underline{41}$ of excess entropy production. Since we expect $\mu$ to be of order the weak scale whereas the VEV of $z$ is of order the Planck scale, the potential is very flat. This means that if $z$ is displaced from its minimum after inflation (and we would expect an $\mathcal{O}\left(M_{P}\right)$ displacement), the subsequent evolution of $z$ would lead to huge entropy generation. The problem appears when the $z$ field begins oscillating about its minimum, which occurs when the Hubble parameter drops to $H \sim m_{z} \sim \mu$, where $m_{z}$ corresponds to $m_{A}$ or $m_{B}$ in Eq. (16). At this time, the Universe becomes matterdominated by Polonyi oscillations until they decay when $H \sim \Gamma_{z} \sim m_{z}^{3} / M_{P}^{2}$. This leads to late reheating and an entropy increase by a factor $M_{P} / \mu \sim 10^{16}$. Furthermore, the late decay almost inevitably leads to an overproduction of cold dark matter in the form of the lightest supersymmetric particle (LSP) ${ }^{42]}$ We note, however, that the Polonyi problem can be alleviated by a mechanism of strong stabilization, 43 as discussed in Section 10 below.

The gravitino poses other cosmological problems for supergravity models of cosmology, stemming from the abundance of gravitinos produced after inflation. 44 If the gravitino is the LSP, the relic gravitino abundance could exceed the permitted density of cold dark matter, depending on its mass. On the other hand, if the gravitino is not the LSP, the fact that the gravitino couplings to other particles are Planck-suppressed implies that its lifetime for decays into other particles may be quite long: $\tau \sim M_{P}^{2} / m_{3 / 2}^{3}$. Also in this case the mass and abundance of the gravitino are constrained, by experimental limits on late-decaying particles, particularly from

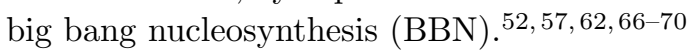

It is easy to break supersymmetry in no-scale supergravity. Even in the minimal $\mathrm{SU}(1,1) / \mathrm{U}(1)$ case, simply taking a constant superpotential, $W=\mu$, leads to a non-zero gravitino mass: d

$$
m_{3 / 2}=\frac{\mu}{\left(T+T^{*}\right)^{3 / 2}},
$$

whereas the scalar potential vanishes (as in Eq. (7)). Hence the magnitude of the gravitino mass is undetermined so long as the modulus $T$ remains unfixed. On the other hand, in this case there is no supersymmetry breaking in the matter sector:

$$
m_{0}=0, \quad B_{0}=0, \quad A_{0}=0 .
$$

We discuss later other mechanisms for breaking supersymmetry in the matter sectors of no-scale models, and how the Polonyi and gravitino problems may be avoided.

${ }^{\mathrm{d}}$ We point out that simply adding a constant superpotential in minimal supergravity does not break supersymmetry. Rather, minimization of the potential in this case leads to a supersymmetrypreserving AdS vacuum. 


\section{Introduction to Inflation in Supergravity}

\subsection{Basic Principles of Models of Inflation}

Before focusing on supersymmetric models of inflation, we first review briefly the dynamics of single-field models of inflation.

The contribution to the energy-momentum tensor of the scalar field $\phi$ is

$$
T_{\mu \nu}=\partial_{\mu} \phi \partial_{\nu} \phi-\frac{1}{2} g_{\mu \nu} \partial_{\rho} \phi \partial^{\rho} \phi+g_{\mu \nu} V(\phi) .
$$

Assuming that it may be treated as a perfect fluid, we can express as follows the energy density $\rho$ and pressure $p$ due to the scalar $\phi$ :

$$
\begin{aligned}
\rho & =\frac{1}{2} \dot{\phi}^{2}+\frac{1}{2} R^{-2}(t)(\nabla \phi)^{2}+V(\phi), \\
p & =\frac{1}{2} \dot{\phi}^{2}-\frac{1}{6} R^{-2}(t)(\nabla \phi)^{2}-V(\phi),
\end{aligned}
$$

where $R(t)$ is the cosmological scale factor. Ignoring the spatial-gradient terms (which is appropriate as the Universe becomes almost homogeneous as it expands), the equation of motion of $\phi$ obtained from the conservation of $T_{\mu \nu}$ is

$$
\ddot{\phi}+3 H \dot{\phi}+\frac{\partial V}{\partial \phi}=0
$$

where $H=\dot{R} / R$ is the Hubble parameter. If we approximate $\partial V / \partial \phi \simeq$ $\left(\partial^{2} V / \partial \phi^{2}\right) \phi=m^{2}(\phi) \phi$, the solution to the equation of motion when $\left|m^{2}\right| \ll H^{2}$ is $\phi \sim \exp \left(\left|m^{2}\right| t / 3 H\right)$, and the field evolves very slowly over a time period $H \tau \sim 3 H^{2} /\left|m^{2}\right|$, during which the universe expands (near-)exponentially. Enough inflation is obtained if the potential is sufficiently flat, i.e., $\left|\mathrm{m}^{2}\right|$ is small enough.

\subsection{Simple Supergravity Models of Inflation}

Restricting our attention to theories of inflation in the context of supersymmetry, for the reasons discussed earlier we focus on supergravity models. These include some Planck-scale effects which may be important for inflation and, as already discussed, make possible the breaking of supersymmetry while (almost) cancelling the cosmological constant.

We begin with the simplest such model, which is based on a single chiral superfield, $\phi$, the inflaton, $\frac{46}{6}$ in minimal supergravity with $K=\phi \phi^{*}$. One can consider a general polynomial form for the superpotential, ${ }^{27}$ the simplest being ${ }^{\sqrt{71}}$

$$
W=M^{2}(1-\phi)^{2}
$$

which leads to

$$
\begin{aligned}
V= & M^{4} e^{|\phi|^{2}}\left[1+|\phi|^{2}-\left(\phi^{2}+\phi^{* 2}\right)-2|\phi|^{2}\left(\phi+\phi^{*}\right)+5|\phi|^{4}\right. \\
& \left.+|\phi|^{2}\left(\phi^{2}+\phi^{* 2}\right)-2|\phi|^{4}\left(\phi+\phi^{*}\right)+|\phi|^{6}\right] .
\end{aligned}
$$


This can be expanded about the origin in the real direction $\phi=\phi^{*}$ to give

$$
V \simeq M^{4}\left(1-4 \phi^{3}+\frac{13}{2} \phi^{4}+\ldots\right),
$$

which is shown in Fig. 2. This is an example of new inflation ${ }^{26}$ driven by the cubic term, and the mass scale $M \sim 10^{-4}$ is determined by normalization of the CMB fluctuation spectrum. ${ }^{9}$ We note that, although the theory defined by 27 is constructed to avoid the $\eta$-problem, a generic inflationary model is in general plagued by the problem of large masses. This simple model is an example of "accidental" inflation, ${ }^{72}$ as the ratio of the constant and linear terms in 27) must be equal to 1 to very high accuracy in order to avoid the $\eta$-problem.

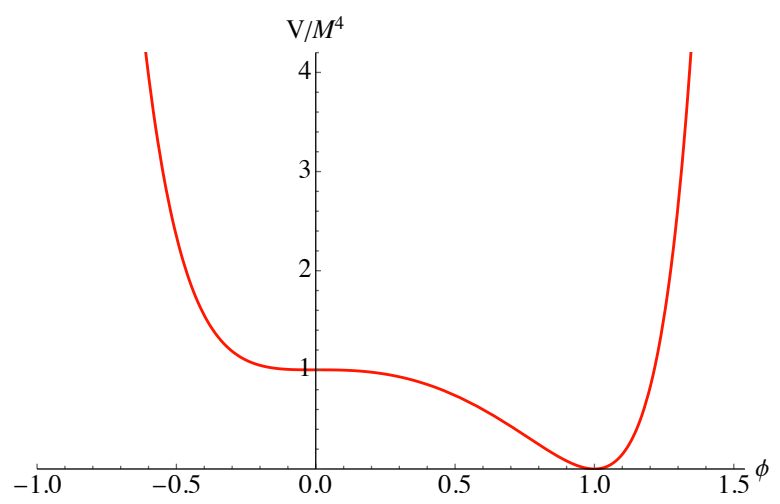

Fig. 2. The inflaton potential in a simple mode 71 based on minimal supergravity.

It is possible to construct many more examples of inflationary models by adding an auxiliary chiral field, $S \stackrel{73+75}{ }$ Consider, for example

$$
K=S S^{*}-\frac{1}{2}\left(\phi-\phi^{*}\right)^{2},
$$

which can still be viewed as minimal because $K_{j}^{i}=\delta_{j}^{i}$. Then, for the simple choice

$$
W=S f(\phi)
$$

one finds

$$
V=|f(\phi)|^{2}
$$

for $S=\operatorname{Im} \phi=0$, and one can easily generate any scalar potential that is a perfect square.

Given the relative ease of constructing inflationary models in minimal supergravity, it is natural to ask about the possibilities in the context of no-scale supergravity $\left.{ }^{76}+79\right]$ One interesting example is given by

$$
W=M^{2}\left(\phi-\phi^{4} / 4\right),
$$


which gives

$$
\hat{V}=M^{4}\left|1-\phi^{3}\right|^{2},
$$

a potential that is very similar to that shown in Fig. 2 .

\section{Inflationary Observables}

Quantum fluctuations during inflation generate scalar density and tensor metric perturbations that leave imprints on the CMB and large-scale structure 6 6 The overall scale of the potential is related to the amplitude, $A_{s}$, of the power spectrum of scalar perturbations in the $\mathrm{CMB}, \frac{10}{10}$ and other $\mathrm{CMB}$ observables include the tilt in the spectrum of scalar perturbations, $n_{s}$, and the tensor-to-scalar ratio, $r$. It is convenient to characterize these in terms of the slow-roll parameters $\epsilon$ and $\eta \cdot{ }^{2]}$ For a given single-field scalar potential, these are given by

$$
\epsilon \equiv \frac{1}{2} M_{P}^{2}\left(\frac{V^{\prime}}{V}\right)^{2} ; \eta \equiv M_{P}^{2}\left(\frac{V^{\prime \prime}}{V}\right)
$$

where, here and subsequently, the prime denotes a derivative with respect to the inflaton field $\phi$. The tilt in the spectrum of scalar perturbations, $n_{s}$, and the tensorto-scalar ratio, $r$ are the principal CMB observables, 10 and can be expressed as follows in terms of the slow-roll parameters at the pivot scale $k_{*}=0.05 \mathrm{Mpc}^{-1}$ :

$$
\begin{aligned}
\text { Amplitude of scalar perturbations } A_{s}: A_{s} & =\frac{V_{*}}{24 \pi^{2} \epsilon_{*} M_{P}^{4}} \simeq 2.1 \times 10^{-9}, \\
\text { Scalar spectral tilt } n_{s}: n_{s} & \simeq 1-6 \epsilon_{*}+2 \eta_{*} \\
& =0.965 \pm 0.004(68 \% \mathrm{CL}), \\
\text { Tensor-to-scalar ratio } r: r & \simeq 16 \epsilon_{*}<0.061(95 \% \mathrm{CL}) .
\end{aligned}
$$

The region of the $\left(n_{s}, r\right)$ plane allowed by the Planck data is shown in Fig. 3 In addition to the above expressions, we note that the number of $e$-folds, $N_{i}$, of inflation between the initial and final values of the inflaton field $\phi_{i, \text { end }}$ is given by the formula

$$
N_{i} \equiv \ln \left(\frac{R_{\text {end }}}{R_{i}}\right)=\int_{t_{i}}^{t_{\text {end }}} H d t \simeq-\int_{\phi_{i}}^{\phi_{\text {end }}} \frac{1}{\sqrt{2 \epsilon}} \frac{d \phi}{M_{P}} .
$$

The number of $e$-folds between when the scale $k_{*}$ exits the horizon and the end of inflation is denoted by $N_{*}$. Typical values of $N_{*}$ are in the range $\sim 50-60$, dependent on the mechanism ending inflation $10,80 \mid 81$

We can now test the simple models of inflation discussed in the previous Section. For the potential determined by Eq. (27), the amplitude of density fluctuations 36 ) implies that $M \sim 10^{-4} M_{P}$, as expected. In this case the slow-roll parameter $\epsilon \simeq$ $3.6 \times 10^{-10}$ is very small, yielding a value of the tensor-to-scalar ratio that is allowed, but unobservably small. However, the scalar tilt in this model is $n_{s} \simeq 1+2 \eta \simeq 0.928$, which is strongly excluded by the Planck data 10 Similarly, the no-scale potential defined by (33) gives $\epsilon \simeq 1.5 \times 10^{-9}$, with similar values of $M$ and $\eta$, resulting again 
in $n_{s} \simeq 0.928$, excluding the model. This value of $n_{s}$ does not even lie with the range shown in Fig. 3

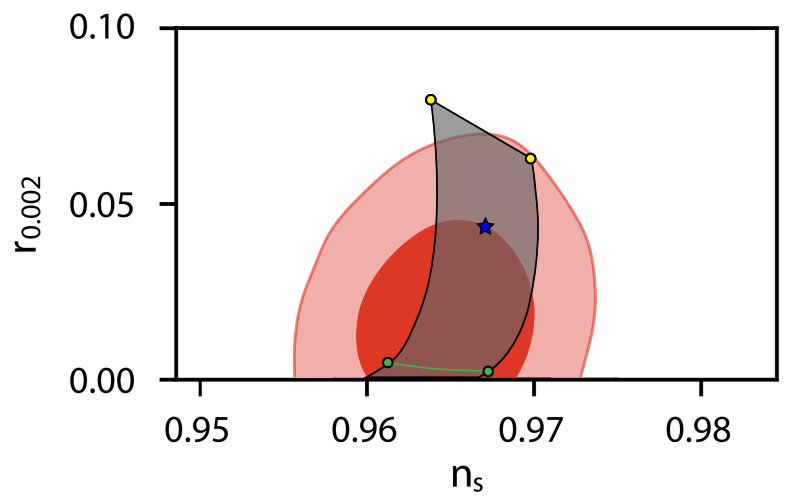

Fig. 3. Plot of the CMB observables $n_{s}$ and $r$. The red shadings correspond to the $68 \%$ and $95 \%$ confidence level regions from Planck data combined with BICEP2/Keck results!10 The pairs of dots are the predictions of the $\alpha$-Starobinsky potential $(106)$ discussed later for $N_{*}=50$ (left) and 60 (right). The upper (lower) pair of yellow (green) dots are the predictions when $\alpha=100$ $(\alpha=1$, corresponding to the Starobinsky model (40)), while the lower end of the swath represents the cosmological observables in the limit $\alpha \rightarrow 0$. The 68\% upper bound $r_{0.002} \lesssim 0.041$, indicated by the blue star, is attained for $\alpha \sim 27$ when $n_{s} \sim 0.967$, for a nominal choice of $N_{*} \simeq 55$.

In contrast to the models discussed above, one of the first models of inflation, namely the Starobinsky model,$\frac{3}{3}$ yields a value of $n_{s}$ that is in excellent agreement with observation, and a value of $r$ that is testable in the next generation of CMB experiments. As originally written, the model was based on an $R+R^{2}$ theory of gravity. However, a suitable conformal transformation ${ }^{4}$ brings the theory into the form of a theory with an Einstein-Hilbert action for gravity, and a canonical scalar field with a scalar potential of the form

$$
V=3 M^{2} e^{-\sqrt{2 / 3} \phi} \sinh ^{2}(\phi / \sqrt{6})=\frac{3}{4} M^{2}\left(1-e^{-\sqrt{2 / 3} \phi}\right)^{2},
$$

as depicted in Fig. 4 .

This model was the first to predict a slightly red scalar perturbation spectrum $\left(n_{s}<1\right){ }^{6}$ It is easy to determine in analytic form the slow-roll parameters for this potential: $: 20$

$$
\begin{aligned}
A_{s} & =\frac{3 M^{2}}{8 \pi^{2}} \sinh ^{4}(\phi / \sqrt{6}), \\
\epsilon & =\frac{1}{3} \operatorname{csch}^{2}(\phi / \sqrt{6}) e^{-\sqrt{2 / 3} \phi}, \\
\eta & =\frac{1}{3} \operatorname{csch}^{2}(\phi / \sqrt{6})\left(2 e^{-\sqrt{2 / 3} \phi}-1\right) .
\end{aligned}
$$

For $N_{*}=55$, we find $M=1.25 \times 10^{-5} M_{P}, n_{s}=0.965$, and $r=0.0035$. The line between the two green dots in Fig. 3 corresponds to the predictions of the 


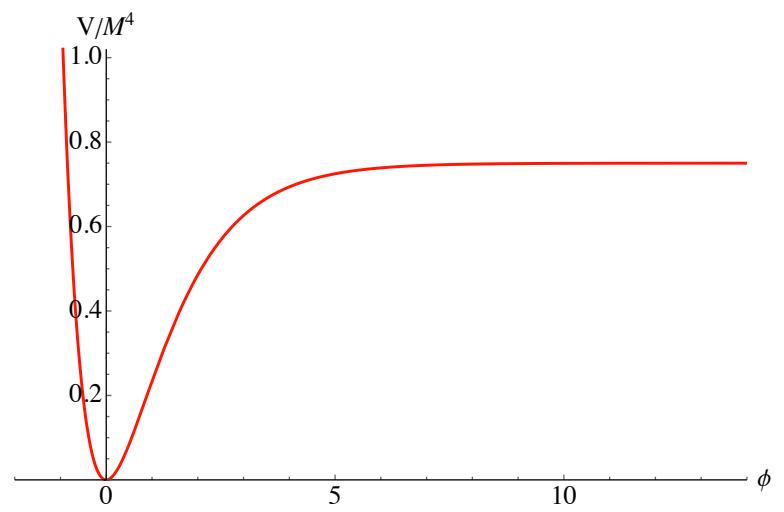

Fig. 4. The effective scalar potential in the Starobinsky model of inflation! 3

Starobinsky model for $N_{*}=50$ to 60 . We make the connection between this model and no-scale supergravity in the next Section.

\section{A No-Scale Starobinsky model}

As already mentioned, the Starobinsky inflationary model based on the potential in Eq. 40 had its origins in higher-derivative gravity. It can, however, be seen to arise rather simply and directly within the no-scale supergravity framework. ${ }^{20}$

Our starting point is the no-scale supergravity scalar potential given in Eqs. (10) and (11). We see immediately that it is not possible to construct a Starobinskylike model using only a single field, $T$. In that case, the scale invariance of the Starobinsky potential at large field values would require a constant potential at large $T$, which is possible only if the superpotential scales as $W \sim T^{3 / 2}{ }^{82} \mathrm{In}$ that case, however, the leading term in $\hat{V}$ would be negative, $\hat{V} \sim-\frac{3}{2} T^{2}$. Therefore a minimal model requires two fields, which we take as $T$ and the inflaton, $\phi$.

For now, we assume that the modulus is fixed by some unspecified mechanism with $\langle T\rangle=1 / 2$ for illustration (the value of $\langle T\rangle$ is unimportant and its stabilization is discussed in Section 10 below). Further, we postulate the following Wess-Zumino form for the superpotential20

$$
f(\phi)=\frac{M}{2} \phi^{2}-\frac{\lambda}{3} \phi^{3}
$$

which is a function of $\phi$ alone. In this case, $W_{T}=0$, and we see from Eq. (11) that $\hat{V}=\left|W_{\phi}\right|^{2}$. The resulting potential is

$$
V(\phi)=M^{2} \frac{|\phi|^{2}|1-\lambda \phi / M|^{2}}{\left(1-|\phi|^{2} / 3\right)^{2}} .
$$

We can rewrite the kinetic terms in Eq. (9) as

$$
\mathcal{L}_{K E}=\left(\partial_{\mu} \phi^{*}, \partial_{\mu} T^{*}\right)\left(\frac{3}{\left(T+T^{*}-|\phi|^{2} / 3\right)^{2}}\right)\left(\begin{array}{cc}
\frac{\left(T+T^{*}\right)}{3}-\phi / 3 \\
-\phi^{*} / 3 & 1
\end{array}\right)\left(\begin{array}{c}
\partial^{\mu} \phi \\
\partial^{\mu} T
\end{array}\right),
$$


indicating that neither $T$ nor $\phi$ is normalized canonically. When $T$ is fixed, we can define the canonically-normalized field $\chi$ :

$$
\chi \equiv \sqrt{3} \tanh ^{-1}\left(\frac{\phi}{\sqrt{3}}\right) .
$$

Decomposing $\chi$ into its real and imaginary parts: $\chi=(x+i y) / \sqrt{2}$, we find that the potential is minimized for $y=0$, and that the potential in the real direction takes the same form as the Starobinsky potential in Eq. 40 when $\lambda=M / \sqrt{3}$.

To get a feel for how "accidental" this result is, the potential is plotted for several values of $\lambda / M \simeq 1 / \sqrt{3}$ in Fig. 5. Requiring $N_{*}=50$ to $60 e$-folds specifies the value of the field $x$ at the beginning of inflation. For example, the nominal choice $N_{*}=55$ corresponds to $x=5.35$ and, as one can see in Fig. 5 , inflation is still possible for $\sqrt{3} \lambda / M$ slightly less than 1 . However, deviations from 1 by more than a few parts in $10^{-4}$ would not provide a suitable inflationary potential, as seen in Fig. 6 .

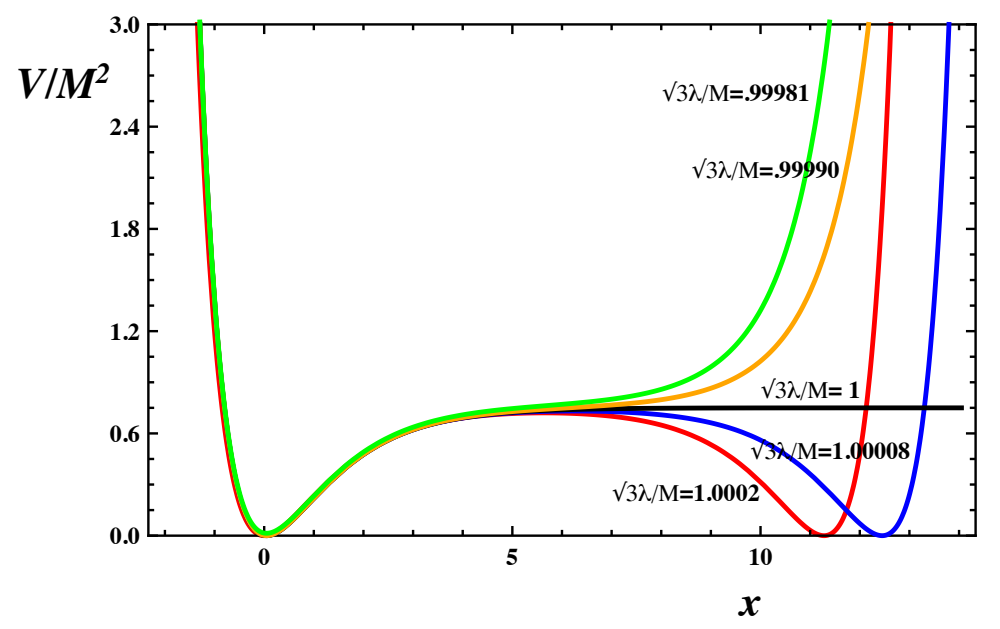

Fig. 5. Starobinsky-like inflationary potential in the no-scale supergravity model with superpotential (44) for choices of $\lambda \sim M / \sqrt{3}$, as indicated.

Fig. 6 displays the predictions for $\left(n_{s}, r\right)$ in this model for five choices of the coupling $\lambda$ that yield $n_{s} \in[0.93,1.00]$ and $N_{*} \in[50,60]$. The last 50 to $60 e$-folds of inflation arise as $x$ rolls to zero from $\sim 5.1-5.8$, with the exact value depending on $\lambda$ and $N_{*}$. We see again that the values of $\lambda$ are constrained to be close to the critical value $M / \sqrt{3}$, for which we find extremely good agreement with the Planck determination of $n_{s}$. The values of $r$ are well below the current experimental limit, 11 varying over the range $0.0012-0.0084$, in the models displayed, with $r \simeq 0.003$ for $\lambda=M / \sqrt{3}$.

We turn in later Sections to some more formal aspects of no-scale supergravity and its relationship to higher-derivative gravity and the Starobinsky model, to some 


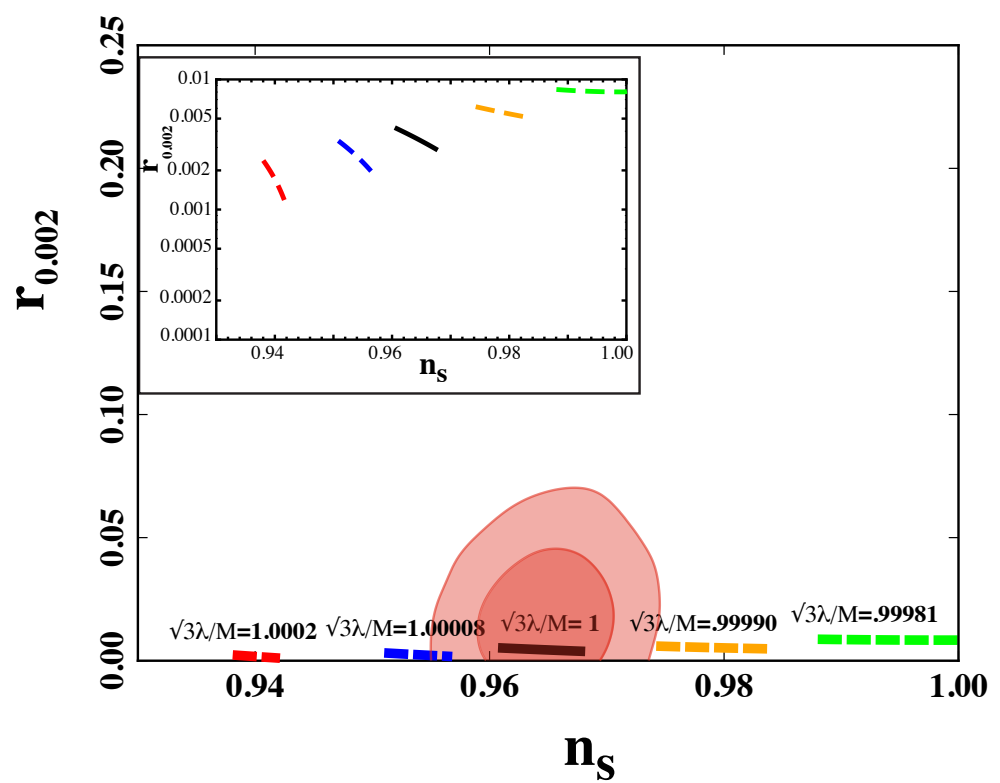

Fig. 6. Predictions for the tilt $n_{s}$ in the spectrum of scalar perturbations and for the tensorto-scalar ratio $r$, compared with the 68 and $95 \%$ CL regions from Planck data combined with BICEP2/Keck results.10 In the main panel the lines are labelled by the values of $\sqrt{3} \lambda / M$ (in Planck units) assumed in each case. In the inset, the same cases are shown on a log scale to display more clearly the values of $r$.

generalizations of the superpotential (44) and to aspects of the phenomenology of no-scale models of inflation. However, we comment first how modifying the no-scale Kähler potential can affect the observables, causing deviations from the Starobinsky predictions .82

The Starobinsky potential can be expressed in the simple form

$$
V=A\left(1-e^{-B x}\right)^{2},
$$

with $B=\sqrt{2 / 3}$, and we note that the inflationary predictions are derived in the large-field regime where the leading non-constant term is $\propto e^{-B x}$. We considered in Ref. 82 phenomenological generalizations of 480 in which

$$
V=A\left(1-\delta e^{-B x}+\mathcal{O}\left(e^{-2 B x}\right)\right)
$$

with $\delta$ and $B$ treated as free parameters that may deviate from the Starobinsky values $\delta=2$ and $B=\sqrt{2 / 3}$. At leading order when $e^{-B x}$ is small, one finds

$$
\begin{aligned}
n_{s} & =1-2 B^{2} \delta e^{-B x}+\cdots, \\
r & =8 B^{2} \delta^{2} e^{-2 B x}+\cdots \\
N_{*} & =\frac{1}{B^{2} \delta} e^{+B x}+\cdots
\end{aligned}
$$


and we have

$$
n_{s}=1-\frac{2}{N_{*}}+\cdots, \quad r=\frac{8}{B^{2} N_{*}^{2}}+\cdots .
$$

Requiring $N_{*}=50$ to 60 yields the characteristic predictions $n_{s}=0.960$ to 0.967 , independent of $B$, and the Starobinsky choice $B=\sqrt{2 / 3}$ yields $r=12 / N_{*}^{2}=0.0048$ to 0.0033 . Since the experimental upper limit is $r<0.06 \frac{10}{10}$ it is clear that substantial deviations from the Starobinsky value of $B$ are currently allowed. We note finally that the predictions (51) are independent of $\delta$.

Different values of $B$ may be obtained by considering generalized Kähler potentials $\sqrt{82}$ that include an inflationary sector:

$$
K \ni K_{\mathrm{inf}}=-3 \alpha \ln \left(T+T^{*}\right),
$$

where $\alpha \neq 1$ in general. In such a case

$$
B=\sqrt{\frac{2}{3 \alpha}}
$$

leading to the prediction

$$
r \simeq \frac{12 \alpha}{N_{*}^{2}}
$$

For example, a simple string compactification contains 3 complex moduli $T^{i}$ whose product is the volume modulus $T$ considered above. Inflation might be driven by just one or a pair of the $T^{i}$, in which case $\alpha=1 / 3$ or $2 / 3$. In these examples $r$ is decreased below the Starobinsky prediction, rendering its experimental detection more challenging. However, it is interesting that, within the class of string-inspired no-scale models discussed here, measuring $r$ might cast light on the dynamics of string compactification. 82

One may also consider models with larger values of $\alpha$, as illustrated in Fig. 3 . where we see that values of $\alpha \lesssim 27$ are allowed by the current upper limit on $r$. However, we note that, for large values of $\alpha \gg 1$, sub-asymptotic corrections to the leading-order predictions (50, 51) become important, causing the curvature in the predicted bands in Fig. 3

We discuss such models with $\alpha \neq 1$ in more detail in subsequent Sections.

\section{On the Structure of No-Scale Supergravity}

Motivated by this phenomenological connection to the Starobinsky $R+R^{2}$ model of inflation, we now go beyond the brief introduction to the minimal and no-scale supergravities given in Section 2. We explore some more theoretical aspects of the construction of the supergravity Lagrangian, highlighting particular aspects that will help in connecting no-scale supergravity with $R^{2}$ and $R+R^{2}$ gravity.

As is well known in globally-supersymmetric models, the supersymmetry algebra includes auxiliary fields that can be removed using their equations of motion. The 
Lagrangian can be written as

$$
\mathcal{L}=\mathcal{L}_{\text {aux }}+\mathcal{L}^{\prime}
$$

with

$$
\begin{aligned}
\mathcal{L}_{\text {aux }}= & \frac{9}{\Phi}\left|\frac{1}{2} g^{*}+\cdots\right|^{2} \frac{3}{\Phi J_{\phi \phi^{*}}}\left|\frac{1}{2} g^{*}\left(\frac{g_{\phi^{*}}^{*}}{g^{*}}-J_{\phi^{*}}\right)+\cdots\right|^{2} \\
& -\frac{1}{4 \Phi}\left[\left(\Phi_{\phi^{*}} \partial_{\mu} \phi^{*}-\Phi_{\phi} \partial_{\mu} \phi\right)+\cdots\right]^{2}
\end{aligned}
$$

and

$$
\mathcal{L}^{\prime}=-\Phi_{\phi \phi^{*}}\left|\partial_{\mu} \phi\right|^{2}-\frac{1}{6} \Phi R+\cdots
$$

In writing this greatly abbreviated version of the Lagrangian, we have dropped all terms involving fermions (including the gravitino) and vectors, keeping only the purely scalar part of the Lagrangian coupled to the gravitational curvature $R$. E Here, the fields $\phi^{i}$ correspond to scalars though, for clarity, we suppress the index $i$, and $J=3 \ln (\Phi / 3)$. As we will see, the function $J$ can be identified (up to a sign) as the Kähler potential: $J=-K$, while $g$ is related to the superpotential.

We note that the Lagrangian written this way does not correspond to minimal Einstein gravity, as it contains a coupling of $R$ to the function $\Phi$. We will return to this form later when we consider higher-order theories of gravity. We can perform a conformal transformation of the metric: $g_{\mu \nu} \rightarrow e^{2 \Omega} g_{\mu \nu}$, with $e^{2 \Omega}=\frac{1}{3} \Phi$, corresponding to $J=6 \Omega$. Thus we can bring the curvature term into its Einstein-Hilbert form by making a conformal transformation proportional to the Kähler potential, leading to

$$
-\frac{1}{6} \Phi R \rightarrow-\frac{1}{2} R+\frac{3}{4}\left(\partial_{\mu}(\ln \Phi)\right)^{2} .
$$

We can now combine $\mathcal{L}^{\prime}$ with the final term in $\mathcal{L}_{\text {aux }}$ to write the kinetic terms in the Lagrangian as

$$
\begin{aligned}
\mathcal{L}_{\text {kin }}= & -\frac{1}{2} R+\frac{3}{4}\left(\partial_{\mu}(\ln \Phi)\right)^{2}-\Phi_{\phi \phi^{*}}\left|\partial_{\mu} \phi\right|^{2}\left(\frac{3}{\Phi}\right) \\
& -\frac{1}{4 \Phi}\left(\Phi_{\phi^{*}} \partial_{\mu} \phi^{*}-\Phi_{\phi} \partial_{\mu} \phi\right)^{2}\left(\frac{3}{\Phi}\right),
\end{aligned}
$$

where we have included the appropriate conformal factor in the last two terms. We can then rewrite $(59)$ as

$$
\mathcal{L}_{\text {kin }}=-\frac{1}{2} R-\left(\frac{3}{\Phi} \Phi_{\phi \phi^{*}}-\frac{3}{\Phi^{2}} \Phi_{\phi} \Phi_{\phi^{*}}\right)\left|\partial_{\mu} \phi\right|^{2}=-\frac{1}{2} R-J_{i}^{j} \partial_{\mu} \phi^{i} \partial^{\mu} \phi_{j}^{*}
$$

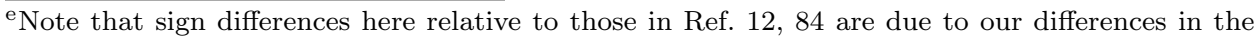
conventions for $R$ and the metric signature. Here, we are using the (+- - ) metric.
} 
The identification of the Kähler potential $K=-J$ allows us to write the kinetic terms in the conventional manner seen in Eq. (1), recalling that the Kähler potential is (up to a constant) the conformal factor: $K=-6 \Omega$.

Next, we consider the remaining highlighted terms in $\mathcal{L}_{\text {aux }}$, which we identify with the scalar potential:

$$
V=\frac{9}{4 \Phi}|g|^{2}\left(\frac{9}{\Phi^{2}}\right)+\frac{3}{4 \Phi} \frac{|g|^{2}}{J_{\phi \phi^{*}}}\left|\frac{g_{\phi}}{g}-J_{\phi}\right|^{2}\left(\frac{9}{\Phi^{2}}\right)
$$

where we have included once again the conformal factor needed to write the potential in the Einstein frame. We define the function

$$
G \equiv-J+\ln \left|\frac{g}{2}\right|^{2}=K+\ln |W|^{2}
$$

where we have associated the function $g$ with the superpotential: $g=2 W$. Then, after some simple algebra we arrive at the expression given in Eq. 22 for the effective scalar potential.

To obtain minimal supergravity, we must choose

$$
\Phi=3 e^{-\phi \phi^{*} / 3},
$$

which corresponds to the Kähler potential given in Eq. (3) and the potential (4). There are of course simpler choices for the function $\Phi$. Of particular interest, will be the class of functions for which there is no kinetic term for scalars in Eq. (59) and hence $\Phi_{\phi \phi^{*}}=0$, as appropriate in $R^{2}$ theories of gravity as we discuss below. One choice of $\phi$ with this property is

$$
\Phi=3\left(\phi+\phi^{*}\right)
$$

corresponding to $K=-3 \ln \left(\phi+\phi^{*}\right)$ as in Eq. (5), after the identification $\phi \rightarrow$ $T$. The scalar potential vanishes as in Eq. (7), which is characteristic of no-scale supergravity $[15]$

\section{No-Scale Supergravity, $R+R^{2}$ Inflation, and de Sitter Solutions}

The parallels between no-scale supergravity and $R+R^{2}$ models of inflation suggest that there must be a deeper connection between them, and there are indeed many such connections with both $R^{2}$ and $R+R^{2}$ gravity models, $83 \mid 84$ as we now discuss. We begin by recalling some basic features of $R^{2}$ gravity and its transformation to Einstein gravity. We then repeat the procedure for $R+R^{2}$ gravity, which leads to the Starobinsky model discussed above. We also consider the addition to these theories of scalar matter and a scalar potential defined in the conformal frame.

The action for a pure, scale-invariant $R^{2}$ theory of gravity can be written as

$$
\mathcal{A}=\frac{1}{2} \int d^{4} x \sqrt{-g} \alpha R^{2}
$$


where $\alpha$ is a dimensionless constant. This action may be rewritten in the following form, by inserting a Lagrange multiplier field $\Phi$ :

$$
\mathcal{A}=-\frac{1}{2} \int d^{4} x \sqrt{-g}\left(2 \alpha \Phi R+\alpha \Phi^{2}\right) .
$$

We recall at this point the form of the Ricci curvature term in the original supergravity Lagrangian in Eq. (57) with $\alpha=1 / 6$. The action (66) may be rewritten in the Einstein-Hilbert form, if one rescales the metric by a conformal factor $\Omega$, as follows:

$$
\tilde{g}_{\mu \nu}=e^{2 \Omega} g_{\mu \nu}=2 \alpha \Phi g_{\mu \nu} .
$$

After the conformal transformation, the curvature can be rewritten as

$$
R=e^{2 \Omega}\left(\tilde{R}-6 \partial^{\mu} \Omega \partial_{\mu} \Omega+6 \square \Omega\right)
$$

and, after eliminating the total divergence, we see that the action in the Einstein frame is:

$$
\mathcal{A}=-\frac{1}{2} \int d^{4} x \sqrt{-\tilde{g}}\left(\tilde{R}-6 \partial^{\mu} \Omega \partial_{\mu} \Omega+\frac{1}{4 \alpha}\right),
$$

or equivalently:

$$
\mathcal{A}=-\frac{1}{2} \int d^{4} x \sqrt{-\tilde{g}}\left(\tilde{R}-\frac{3}{2} \frac{\partial^{\mu} \Phi \partial_{\mu} \Phi}{\Phi^{2}}+\frac{1}{4 \alpha}\right) .
$$

After a field redefinition: $\phi \equiv \sqrt{6} \Omega=\sqrt{\frac{3}{2}} \ln 2 \alpha \Phi$, the action now takes the simple form

$$
\mathcal{A}=-\frac{1}{2} \int d^{4} x \sqrt{-\tilde{g}}\left(\tilde{R}-\partial^{\mu} \phi \partial_{\mu} \phi+\frac{1}{4 \alpha}\right) .
$$

This is a well-known result $\frac{85}{87}$ Pure $R^{2}$ gravity is equivalent to the conventional Einstein-Hilbert theory with a massless scalar field $\phi$ and a cosmological constant $\Lambda=1 / 8 \alpha$. Thus, the dimensionless parameter $\alpha$ in $(65)$ specifies the magnitude of $\Lambda$ in Planck units.

We can extend this discussion to include the Starobinsky model of inflation,, 3 which was formulated by adding the conventional linear Einstein-Hilbert term to the pure $R^{2}$ action 65:

$$
\mathcal{A}=\int d^{4} x \sqrt{-g}\left(-\frac{1}{2} R+\frac{\alpha}{2} R^{2}\right)
$$

We introduce once again a Lagrange multiplier: $-\alpha R^{2} \rightarrow 2 \alpha \Phi R+\alpha \Phi^{2}$ and perform a conformal transformation $4 \sqrt{88}$

$$
\tilde{g}_{\mu \nu}=e^{2 \Omega} g_{\mu \nu}=(1+2 \alpha \Phi) g_{\mu \nu},
$$

finding

$$
\mathcal{A}=-\frac{1}{2} \int d^{4} x \sqrt{-\tilde{g}}\left[\tilde{R}-\frac{6 \alpha^{2}}{(1+2 \alpha \Phi)^{2}}\left(\partial^{\mu} \Phi \partial_{\mu} \Phi-\frac{\Phi^{2}}{6 \alpha}\right)\right]
$$


Setting $\phi \equiv \sqrt{3 / 2} \ln (1+2 \alpha \Phi), \sqrt{72}$ may be written in the Einstein frame as:

$$
\mathcal{A}=-\frac{1}{2} \int d^{4} x \sqrt{-\tilde{g}}\left[\tilde{R}-\partial^{\mu} \phi \partial_{\mu} \phi+\frac{1}{4 \alpha}\left(1-e^{-\sqrt{\frac{2}{3}} \phi}\right)^{2}\right] .
$$

One recovers the inflationary potential 40 with $\alpha=1 / 6 M^{2}$. The scale invariance of the pure $R^{2}$ theory 65 is broken by the Einstein-Hilbert term in 72 , and leads to an effective potential (40) with a constant, scale-invariant asymptotic limit that is approached exponentially at a rate controlled by the Planck scale.

We now introduce $N-1$ additional complex fields $\phi^{i}$ with conformal couplings to $R$ :

$$
\mathcal{A}=-\frac{1}{2} \int d^{4} x \sqrt{-g}\left[\delta R-\alpha R^{2}-2\left(\partial^{\mu} \phi^{i} \partial_{\mu} \phi_{i}^{*}+\frac{1}{6}\left|\phi^{i}\right|^{2} R\right)\right],
$$

where $\delta=0$ corresponds to the $R^{2}$ theory and $\delta=1$ corresponds to the Starobinsky model. Following the same procedure, we transform to the Einstein frame, using a modified conformal factor $\Omega$ :

$$
\tilde{g}_{\mu \nu}=e^{2 \Omega} g_{\mu \nu}=\left(\delta+2 \alpha \Phi-\frac{1}{3} \sum_{i=1}^{N-1}\left|\phi^{i}\right|^{2}\right) g_{\mu \nu},
$$

leading to the following generalization of 69):

$$
\begin{aligned}
\mathcal{A}= & -\frac{1}{2} \int d^{4} x \sqrt{-\tilde{g}}\left[\tilde{R}-6 \partial^{\mu} \Omega \partial_{\mu} \Omega\right. \\
& \left.-\sum_{i=1}^{N-1} \frac{2 \partial^{\mu} \phi^{i} \partial_{\mu} \phi_{i}^{*}}{\left(\delta+2 \alpha \Phi-\frac{1}{3}\left|\phi^{i}\right|^{2}\right)}+\frac{\alpha \Phi^{2}}{\left(\delta+2 \alpha \Phi-\frac{1}{3}\left|\phi^{i}\right|^{2}\right)^{2}}\right],
\end{aligned}
$$

which may be compared with the effective action of $\mathrm{SU}(\mathrm{N}, 1) / \mathrm{SU}(\mathrm{N}) \times \mathrm{U}(1)$ no-scale supergravity.

First, we notice that the coefficients of the curvature terms in Eqs. (57) and (66) match for $\alpha=1 / 6$. In the case of the $\mathrm{SU}(1,1) / \mathrm{U}(1)$ no-scale supergravity model, $2 \alpha \Phi \rightarrow \Phi / 3 \rightarrow\left(T+T^{*}\right)$, resulting in an equivalence in all kinetic terms, though bearing in mind that all the scalar fields in the supergravity theory are complex, and the association is only possible along the real direction. The second term in Eq. (66) is effectively a potential term, which we see in the Einstein frame is a constant, $1 / 8 \alpha$. In the supergravity context, we are required therefore to add a superpotential term such as

$$
W=T^{3}-\frac{1}{12 \alpha},
$$

which, using Eqs. (10) and (11), generates a scalar potential of the form

$$
V\left(T, T^{*}\right)=\frac{1}{4 \alpha} \frac{T^{2}+T^{* 2}}{\left(T+T^{*}\right)^{2}}
$$

that reduces to a constant $(1 / 8 \alpha)$ along the real direction. We return below to the question of generating de Sitter solutions in no-scale supergravity. 
In the case of the $R+R^{2}$ theory, the correspondence becomes $1+2 \alpha \Phi \rightarrow$ $\Phi / 3 \rightarrow\left(T+T^{*}\right)$ and, once again, a superpotential must be added to generate the Starobinsky potential, as will be discussed in detail below.

Finally, when matter fields are conformally coupled to curvature as in Eq. 766, we must compare Eq. (78) and Eqs. (99)-(11), recalling that the comparison can only be made along the real direction in the supergravity Lagrangian. Terms related to the imaginary parts of fields can also be accounted for by incorporating additional terms in the gravitational action ${ }^{84}$ The kinetic terms are easily seen to be identical, with the replacements $-6 \Omega \rightarrow K$ and $(\delta+2 \alpha \Phi) \rightarrow\left(T+T^{*}\right)$. With these identifications, the conformal factors in front of the matter kinetic terms are equal, $e^{K / 3}$, as is the conformal factor in front of the potential, $e^{2 K / 3}$.

\section{Generalized No-Scale Models, Minkowski Pairs and (Anti-)de Sitter Solutions}

The original no-scale framework describes a non-compact coset field space with constant Kähler curvature, $R=2 / 3$ (we use the convention that $R>0$ for a hyperbolic manifold and $R<0$ for a spherical manifold). As mentioned in Section 5 , this can be generalized by considering a Kähler potential of the form

$$
K=-3 \alpha \ln \left(T+T^{*}\right),
$$

which also parametrizes a non-compact $\mathrm{SU}(1,1) / \mathrm{U}(1)$ coset manifold, but with a constant curvature $R=\frac{2}{3 \alpha}$ that is positive if we assume that $\alpha>0$. This unique structure was first discussed in 1984 in Ref. 16, and similar models have been studied more recently, ${ }^{89}\left[\frac{96}{6}\right.$ where they were termed $\alpha$-attractors.

The de Sitter solution given by Eq. 779 is a special case of a more general class of superpotentials that were also first derived in Ref. 16 .

$$
\begin{array}{ll}
\text { 1) } & W=\lambda \quad \text { with } \quad \alpha=1, \\
\text { 2) } & W=\lambda T^{3 \alpha / 2}, \\
\text { 3) } & W=\lambda T^{3 \alpha / 2}\left(T^{3 \sqrt{\alpha} / 2}-T^{-3 \sqrt{\alpha} / 2}\right),
\end{array}
$$

where we note that 83 corresponds to an AdS space, since $V<0$.

These may be further generalized by considering first a general form for the superpotential that produces a Minkowski solution ${ }^{93}$

$$
W_{M}=\lambda \cdot T^{n_{ \pm}}
$$

where $n_{ \pm}$is given by

$$
n_{ \pm}=\frac{3}{2}(\alpha \pm \sqrt{\alpha}) .
$$

The effective potential vanishes, $V=0$, along the real $T$ direction. Generalizing (84), de Sitter solutions can also be expressed in a compact form, ${ }^{93}, 95$

$$
W_{d S}=\lambda_{1} T^{n_{-}}-\lambda_{2} T^{n_{+}},
$$


where $n_{ \pm}$is again given by (86). Along the real $T$ direction the effective scalar potential 2 is:

$$
V=3 \cdot 2^{2-3 \alpha} \cdot \lambda_{1} \lambda_{2}
$$

giving a de Sitter solution when $\lambda_{1} \lambda_{2}>0$, and AdS when $\lambda_{1} \lambda_{2}<0$. It is fascinating that a de Sitter vacuum construction (87) is obtained by combining two Minkowski vacuum solutions 85 .

This construction based on Minkowski pairs can be generalized to cases with $N>1$ moduli. We first choose

$$
K=-3 \sum_{i=1}^{N} \alpha_{i} \ln \left(\mathcal{V}_{i}\right)
$$

where $\mathcal{V}_{i}=T_{i}+T_{i}^{*}$. Next, we restrict to real values of all the fields, so that $T_{i}=T_{i}^{*} \mathrm{f}$ which leads to:

$$
\mathcal{V}_{i} \longrightarrow \xi_{i}, \quad \text { for } i=1,2, \ldots, N,
$$

with $\xi_{i}=2 T_{i}$. The general $N$-field Minkowski vacuum solutions are given by the following superpotential choice

$$
W_{M}=\lambda \cdot \prod_{i=1}^{N} \xi_{i}^{n_{i}} .
$$

Combining Eq. (2) with the superpotential (91), we find

$$
V=\lambda^{2} \cdot \prod_{i=1}^{N} \xi_{i}^{2 n_{i}-3 \alpha_{i}} \cdot\left(\sum_{i=1}^{N} \frac{\left(2 n_{i}-3 \alpha_{i}\right)^{2}}{3 \alpha_{i}}-3\right),
$$

and it can be seen from Eq. 92 that to find the Minkowski vacuum solutions with $V=0$, we must satisfy the condition

$$
\sum_{i=1}^{N} \frac{\left(2 n_{i}-3 \alpha_{i}\right)^{2}}{3 \alpha_{i}}=3 .
$$

We can parametrize the constraint (93) as a radial unit $N$-vector $\vec{r}=$ $\left(r_{1}, r_{2}, \ldots, r_{N}\right)$, where

$$
r_{i} \equiv \frac{2 n_{i}-3 \alpha_{i}}{3 \sqrt{\alpha_{i}}}, \quad \text { for } i=1,2, \ldots, N .
$$

Combining equations (93) and 94), we find the unit vector condition

$$
\sum_{i=1}^{N} r_{i}^{2}=1
$$

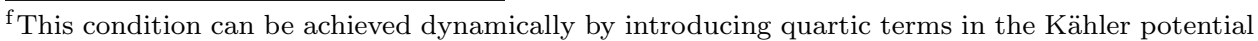
that stabilize the field in the imaginary direction, as we discuss in Section 10
} 
Eq. (94) shows that the $N$-field Minkowski vacuum solutions are parametrized by coordinates compactified on the surface of an $(N-1)$-sphere.

Solving Eq. 947 for $n_{i}$, we find

$$
n_{i}=\frac{3}{2}\left(\alpha_{i}+r_{i} \sqrt{\alpha_{i}}\right), \quad \text { for } i=1,2, \ldots, N,
$$

where $r_{i} \in\{-1,1\}$ and $\alpha_{i}>0$. Once again, dS/AdS solutions are found by taking the difference between two Minkowski solutions:

$$
W_{d S}=\lambda_{1} \cdot \prod_{i=1}^{N} \xi_{i}^{n_{i}}-\lambda_{2} \cdot \prod_{i=1}^{N} \xi_{i}^{\bar{n}_{i}},
$$

where $\bar{n}_{i}=\frac{3}{2}\left(\alpha_{i}+\bar{r}_{i} \sqrt{\alpha_{i}}\right)$, with $\bar{r}_{i}=-r_{i}$. Combining the general $N$-field superpotential form (97) with the effective scalar potential (2), we find the dS/AdS vacuum result

$$
V=12 \lambda_{1} \lambda_{2}
$$

An illustration of these solutions for the case $N=3$ is shown in Fig. 7 .

Minkowski vacuum solutions are described by any point on the surface of the unit sphere. To obtain the dS/AdS vacuum solutions, one can combine any arbitrary point lying on the surface of the sphere with its antipodal point: $\vec{r} \rightarrow-\vec{r}$. In the left panel of Fig. 7, we illustrate four distinct Minkowski vacuum solutions lying on the surface of a sphere which are combined into two unique dS/AdS vacuum solutions. In the right panel of Fig. 7. we show dS/AdS vacuum solutions for possible choices for powers $n_{i}$ (blue sheet) and $\bar{n}_{i}$ (yellow sheet) as functions of the radial vector component $\left|r_{i}\right|$ and curvature parameter $\alpha_{i}$. To recover a Minkowski vacuum solution, one can choose any arbitrary point lying on either the yellow or blue sheet, which yields $V=0$. If we combine the arbitrarily chosen point with a perpendicular point on the opposite sheet (which would correspond to the antipodal point $\vec{r} \rightarrow-\vec{r}$ on the surface of a sphere as shown in the left panel of Fig. 7), it yields the dS/AdS vacuum solution with $V=12 \lambda_{1} \lambda_{2}$.

Finally, we note that these solutions can be further generalized by adding $M-1$ matter fields per modulus as follows. Starting with Eq. (89), we now define

$$
\mathcal{V}_{i}=T_{i}+T_{i}^{*}-\sum_{j=1}^{M-1} \frac{\left|\phi_{i j}\right|^{2}}{3} .
$$

As previously, we then fix the VEVs of the imaginary fields to zero, so that $T_{i}=T_{i}^{*}$ and $\phi_{i j}=\phi_{i j}^{*}$. Using the same notation:

$$
\mathcal{V}_{i} \longrightarrow \xi_{i}, \text { when } T_{i}=T_{i}^{*} \text { and } \phi_{i j}=\phi_{i j}^{*},
$$

the argument inside the logarithm in the Kähler potential becomes

$$
\xi_{i}=2 T_{i}-\sum_{j=1}^{M-1} \frac{\left|\phi_{i j}\right|^{2}}{3} .
$$



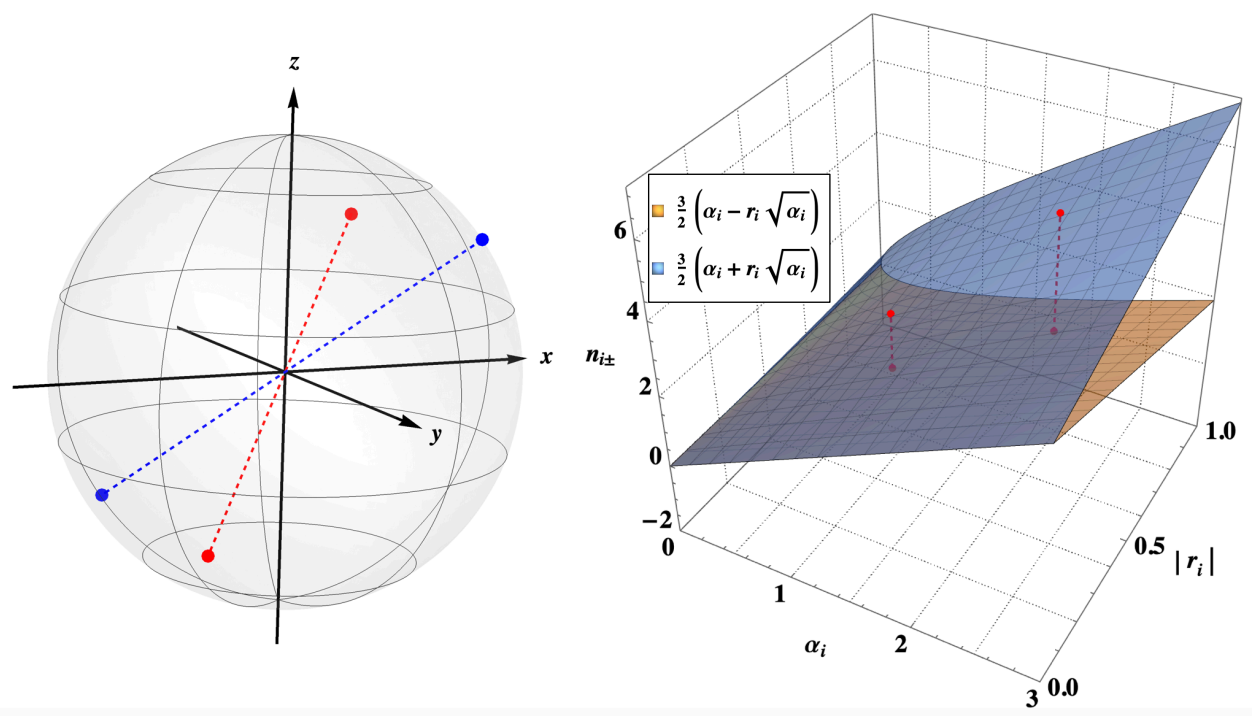

Fig. 7. Left: Illustration of Minkowski pairs on the surface of a sphere. Two distinct Minkowski pairs lie in different octants of the sphere, where the red dots correspond to a Minkowski-pair solution $r=(1 / \sqrt{3},-1 / \sqrt{3}, 1 / \sqrt{3})$ (4th octant) and $\bar{r}=(-1 / \sqrt{3}, 1 / \sqrt{3},-1 / \sqrt{3})$ (6th octant), and the blue dots correspond to a Minkowski-pair solution $r=(1 / \sqrt{3}, 1 / \sqrt{3}, 1 / \sqrt{3})$ (1st octant) and $\bar{r}=(-1 / \sqrt{3},-1 / \sqrt{3},-1 / \sqrt{3})$ (7th octant). Right: Illustration of the Minkowski-pair solution for possible choices for powers $n_{i}$ (blue sheet) and $\bar{n}_{i}$ (yellow sheet). The Minkowski-pair solutions are shown by red dots, and their coordinates, $\left(\alpha_{i}, r_{i}, n_{i}\right)$, are given by $\left(1, \frac{1}{2}, \frac{3}{4}\right)$ with $\left(1, \frac{1}{2}, \frac{9}{4}\right)$ and by $\left(2, \frac{3}{4}, 3-\frac{9}{8} \sqrt{2}\right)$ with $\left(2, \frac{3}{4}, 3+\frac{9}{8} \sqrt{2}\right)$.

With this definition of $\xi_{i}$, Minkowski and dS/AdS vacuum solutions are found using Eq. (97) with the new definition of $\xi_{i}$ in (101).

\section{Generalized No-Scale Starobinsky-Like Inflationary Models}

Following this discussion of the connection between no-scale supergravity and higher-order theories of gravity, we now generalize our previous construction of Starobinsky-like models of inflation in the context of no-scale supergravity.

Assuming an $\mathrm{SU}(2,1) / \mathrm{SU}(2) \times \mathrm{U}(1)$ Kähler potential of the form

$$
K=-3 \alpha \ln \left(T+T^{*}-\frac{|\phi|^{2}}{3}\right)
$$

we consider the following form of superpotential with an arbitrary function $f(\phi) ! 94$

$$
W_{I}=\sqrt{\alpha} f(\phi) \cdot\left(2 T-\frac{\phi^{2}}{3}\right)^{\frac{3}{2}(\alpha-\sqrt{\alpha})} .
$$

This reduces to the following relatively simple form in the real direction $\phi=\phi^{*}$ and 
$T=T^{*}$, for $\langle T\rangle=1 / 2$ :

$$
V=\left(1-\frac{\phi^{2}}{3}\right)^{1-3 \sqrt{\alpha}} \cdot f^{\prime}(\phi)^{2}
$$

where $f^{\prime}(\phi)=d f / d \phi$. Then, if

$$
f^{\prime}(\phi)=\frac{\sqrt{3} M \phi}{(\phi+\sqrt{3})}\left(1-\frac{\phi^{2}}{3}\right)^{(1-3 \sqrt{\alpha}) / 2},
$$

we obtain a Starobinsky-like potential

$$
V=\frac{3}{4} M^{2}\left(1-e^{-\sqrt{\frac{2}{3 \alpha}} \phi^{\prime}}\right)^{2}
$$

after a field redefinition is made to a canonically-normalized field, $\phi^{\prime}$ :

$$
\phi=\sqrt{3} \tanh \left(\frac{\phi^{\prime}}{\sqrt{6 \alpha}}\right) .
$$

This is a concrete realization of the generalization discussed earlier leading to Eq. (53). Some $\alpha$-Starobinsky potential forms with different values of $\alpha$ are plotted in Fig. 8 . We can see in the Figure that increasing the value of the curvature parameter $\alpha$ stretches the Starobinsky potential horizontally, reducing the flatness of the plateau at any fixed value of $\phi^{\prime}$, and thereby increasing $r$ as was seen in Eq. (54).

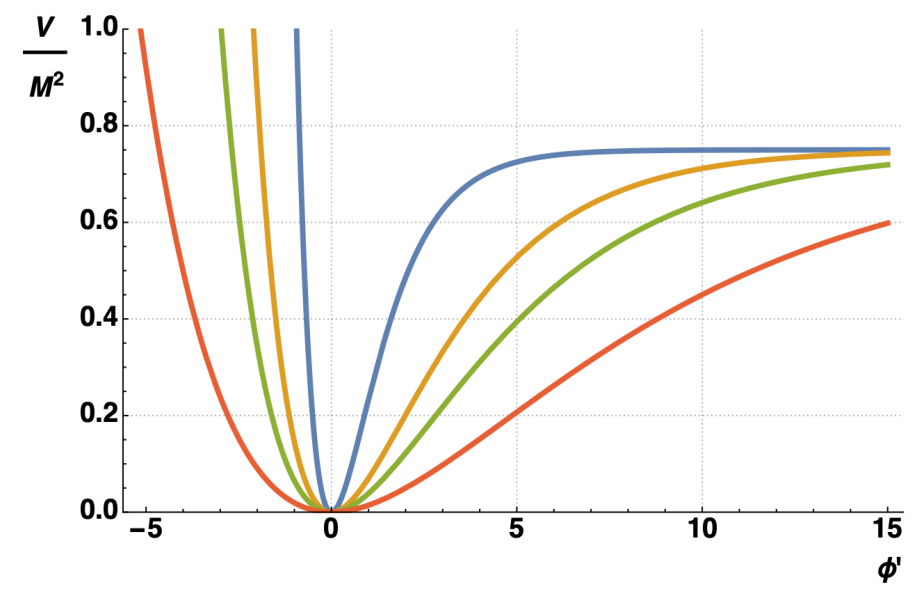

Fig. 8. The $\alpha$-Starobinsky potential (106) for different values of the curvature parameter $\alpha=$ 1 (blue), 5 (yellow), 10 (green), 30 (orange), where $\alpha=1$ corresponds to the original Starobinsky model of inflation (40).

The values of the cosmological observables predicted by these $\alpha$-Starobinsky potentials are shown in the $\left(n_{s}, r\right)$ plane in Fig. 3 , where they can be compared with the results of the Planck collaboration in combination with other CMB data. 
We see that the scalar tilt $n_{s}$ changes only slightly as the curvature parameter $\alpha$ increases, remaining within the range $\sim 0.96-0.97$, whereas the value of the tensor-to-scalar ratio $r(54)$ increases with the value of $\alpha$. The combined CMB data have established a $68 \%$ upper bound on $r_{0.002}$ of $\sim 0.041$, which is reached with $n_{s} \sim 0.967$ when $\alpha \sim 27$ and $N_{*} \simeq 55$, as indicated by the blue star. The prediction of the original Starobinsky model with $N_{*} \in[50,60]$, which corresponds to the case $\alpha=1$, is indicated by the green dots and line at small $r$. Future measurements of $r$ will impose stronger constraints on $\alpha$, and more precise measurements of $n_{s}$ could constrain $N_{*}$ and hence the history of the Universe after inflation, in particular the rate of decay of the inflaton into low-mass particles ${ }^{97}$ (see Fig. 11 below).

The superpotential is found by solving Eq. 105, and has the form of a hypergeometric function for arbitrary $\alpha ! 94$

$$
f(\phi)=M\left[\frac{3-3^{-m}\left(3-\phi^{2}\right)^{m+1}}{2(m+1)}-\frac{\phi^{3}{ }_{2} F_{1}\left(\frac{3}{2},-m ; \frac{5}{2} ; \frac{\phi^{2}}{3}\right)}{3 \sqrt{3}}\right],
$$

where $m=\frac{3}{2}(\sqrt{\alpha}-1)$. Remarkably, the expression in 108 simplifies for particular values of $\alpha$. Specifically, when $\alpha=1, f(\phi)$ is of the Wess-Zumino (WZ) form given in Eq. $44 \sqrt{20}$ with $\lambda=M / \sqrt{3}$, as in this case $m=0$ and ${ }_{2} F_{1}\left(\frac{3}{2}, 0 ; \frac{5}{2} ; \frac{\phi^{2}}{3}\right)=1$. g With this choice, one recovers the exact form of the Starobinsky potential.

The underlying $\mathrm{SU}(2,1) / \mathrm{SU}(2) \times \mathrm{U}(1)$ no-scale symmetry can be used to generate additional forms for the superpotential that lead to the same physical scalar potential, and thereby Starobinsky inflation. For example, we can start from a more symmetric representation of the $\mathrm{SU}(2,1) / \mathrm{SU}(2) \times \mathrm{U}(1)$ coset space $\frac{17}{17}$

$$
K=-3 \ln \left(1-\frac{\left|y_{1}\right|^{2}+\left|y_{2}\right|^{2}}{3}\right)
$$

where the complex fields $y_{1,2}$ are related to the fields $T, \phi$ appearing in 102 for $\alpha=1$ by

$$
y_{1}=\left(\frac{2 \phi}{1+2 T}\right) ; \quad y_{2}=\sqrt{3}\left(\frac{1-2 T}{1+2 T}\right),
$$

with the inverse relations

$$
T=\frac{1}{2}\left(\frac{1-y_{2} / \sqrt{3}}{1+y_{2} / \sqrt{3}}\right) ; \quad \phi=\left(\frac{y_{1}}{1+y_{2} / \sqrt{3}}\right) .
$$

When the coordinates are transformed as in 110, 111, the effective superpotential becomes:

$$
W(T, \phi) \rightarrow \widetilde{W}\left(y_{1}, y_{2}\right)=\left(1+y_{2} / \sqrt{3}\right)^{3} W
$$

\footnotetext{
g The function $f(\phi)$ is a polynomial whenever $9 \alpha$ is an odd perfect square other than 1 .
} 
In the $\left(y_{1}, y_{2}\right)$ basis, one has an effective potential

$$
V=\frac{\hat{V}}{\left(1-\left(\left|y_{1}\right|^{2}+\left|y_{2}\right|^{2}\right) / 3\right)^{2}}
$$

where

$$
\hat{V}=\left|W_{1}\right|^{2}+\left|W_{2}\right|^{2}-\frac{1}{3}\left|3 W-W_{1} y_{1}-W_{2} y_{2}\right|^{2},
$$

with $W_{1,2}=\partial W / \partial y_{1,2}$. If $\left\langle y_{2}\right\rangle=0$, one finds

$$
\begin{aligned}
V & =\frac{\hat{V}}{\left(1-\left|y_{1}\right|^{2} / 3\right)^{2}}, \\
\text { where } \hat{V} & =\left(1-\left|y_{1}\right|^{2} / 3\right)\left|W_{1}\right|^{2}+\left|W_{2}\right|^{2}-3|W|^{2}+\left(y_{1} W_{1} W^{*}+\text { h.c. }\right),
\end{aligned}
$$

and $y_{1}$ can be converted into a canonically-normalized inflaton field $x$ by the transformation

$$
y_{1}= \pm \sqrt{3} \tanh (\chi / \sqrt{3})= \pm \sqrt{3} \tanh (x / \sqrt{6}),
$$

where $\chi=(x+i y) / \sqrt{2}$ and the latter equality holds for $y=0$. From this perspective, one would expect that inflation could be driven by either the matter-like field $\phi$, as in Eq. 44, or the volume modulus, $T$.

The WZ model defined by Eq. (44), can be rewritten in the symmetric basis as 82

$$
W=M\left[\frac{y_{1}^{2}}{2}\left(1+\frac{y_{2}}{\sqrt{3}}\right)-\frac{y_{1}^{3}}{3 \sqrt{3}}\right],
$$

which is a WZ model for $y_{1}$ along with an interaction term $y_{1}^{2} y_{2}$. In this model $W, W_{1}$, and $W_{2}$ are all non-zero, even with the assumption that $y_{2}$ is fixed so that $\left\langle y_{2}\right\rangle=0$. Using 115 we obtain

$$
V=\frac{M^{2}\left|y_{1}\right|^{2}\left|1-y_{1} / \sqrt{3}\right|^{2}}{\left(1-\left|y_{1}\right|^{2} / 3\right)^{2}},
$$

which is of the form needed to yield the Starobinsky potential.

Returning again to the $(T, \phi)$ basis, inflation requires one of the two fields to be fixed. In the WZ-like model (44), we had assumed that $T$ is fixed with a vacuum expectation value of $\operatorname{Re}\langle T\rangle=\frac{1}{2}$ and $\operatorname{Im}\langle T\rangle=0,{ }^{\mathrm{h}}$ in which case the kinetic term becomes:

$$
\mathcal{L}_{\text {kin }}=\frac{1}{\left(1-\frac{\phi \phi^{*}}{3}\right)^{2}} \partial_{\mu} \phi \partial^{\mu} \phi^{*} .
$$

We also assume that the imaginary part of the matter field $\phi$ is fixed to $\operatorname{Im}\langle\phi\rangle=0$ by the dynamics of the potential. The following redefinition leads to a canonicallynormalized field:

$$
\phi= \pm \sqrt{3} \tanh \left(\frac{x}{\sqrt{6}}\right)
$$

${ }^{\mathrm{h}}$ We discuss the question of stabilization in Section 10 below. 
where $x$ is a real scalar field.

Alternatively, we can set $\langle\phi\rangle=0$. The kinetic term in this case is:

$$
\mathcal{L}_{\text {kin }}=\frac{3}{\left(T+T^{*}\right)^{2}} \partial_{\mu} T \partial^{\mu} T^{*} .
$$

We again assume a vacuum expectation value for $T$ with $\operatorname{Im}\langle T\rangle=0$, so that the real part of the volume modulus $T$ can be redefined as a canonically-normalized field given by:

$$
T=\frac{k}{2} e^{ \pm \sqrt{\frac{2}{3}} t},
$$

where the field $t$ is real and the coefficient in front of (121) is compatible with the symmetric field redefinitions (111). In this way, by fixing one of the complex scalar fields $(T, \phi)$ and performing a canonical field redefinition (107) or (121), the $\mathrm{SU}(2,1) / \mathrm{SU}(2) \times \mathrm{U}(1)$ symmetry can be broken into one of four different branches, 98 defined as

Branch I: $\left(\phi=+\sqrt{3 k} \tanh \left(\frac{x}{\sqrt{6}}\right) ;\langle T\rangle=\frac{k}{2}\right) \longrightarrow\left(y_{1}=+\sqrt{3} \tanh \left(\frac{x}{\sqrt{6}}\right) ;\left\langle y_{2}\right\rangle=0\right)$,

Branch II: $\left(\phi=-\sqrt{3 k} \tanh \left(\frac{x}{\sqrt{6}}\right) ;\langle T\rangle=\frac{k}{2}\right) \longrightarrow\left(y_{1}=-\sqrt{3} \tanh \left(\frac{x}{\sqrt{6}}\right) ;\left\langle y_{2}\right\rangle=0\right)$,

Branch III: $\left(\langle\phi\rangle=0 ; T=\frac{k}{2} e^{+\sqrt{\frac{2}{3}} t}\right) \longrightarrow\left(\left\langle y_{1}\right\rangle=0 ; y_{2}=-\sqrt{3} \tanh \left(\frac{t}{\sqrt{6}}\right)\right)$,

Branch IV: $\left(\langle\phi\rangle=0 ; T=\frac{k}{2} e^{-\sqrt{\frac{2}{3}} t}\right) \longrightarrow\left(\left\langle y_{1}\right\rangle=0 ; y_{2}=+\sqrt{3} \tanh \left(\frac{t}{\sqrt{6}}\right)\right)$.

Redefining fields with canonically-normalized kinetic terms in the $\left(y_{1}, y_{2}\right)$ symmetric basis using equations 123$)-(126)$, we may consider a general expression $W\left(y_{1}, y_{2}\right)$ for the superpotential. The $\mathrm{SU}(2,1) / \mathrm{SU}(2) \times \mathrm{U}(1)$ symmetry of the coset space includes the following transformation laws for the fields $y_{1}$ and $y_{2}$.

$$
y_{1} \rightarrow \alpha y_{1}+\beta y_{2}, \quad y_{2} \rightarrow-\beta^{*} y_{1}+\alpha^{*} y_{2} .
$$

The Kähler potential is invariant under these transformations, but the superpotential $W\left(y_{1}, y_{2}\right)$ transforms non-trivially in general. We find it more convenient to use the symmetric $\left(y_{1}, y_{2}\right)$ basis when starting the analysis of a general superpotential. Starting with $W\left(y_{1}, y_{2}\right)$ in any one of the four different branches, the corresponding superpotential in other branches can be obtained by making the transformations (127). The relations between the superpotentials in different branches obtained by these transformations are indicated in Fig. 9.

The general expressions for superpotentials in all the four branches that yield the Starobinsky inflationary potential with canonically-normalized kinetic terms can be 


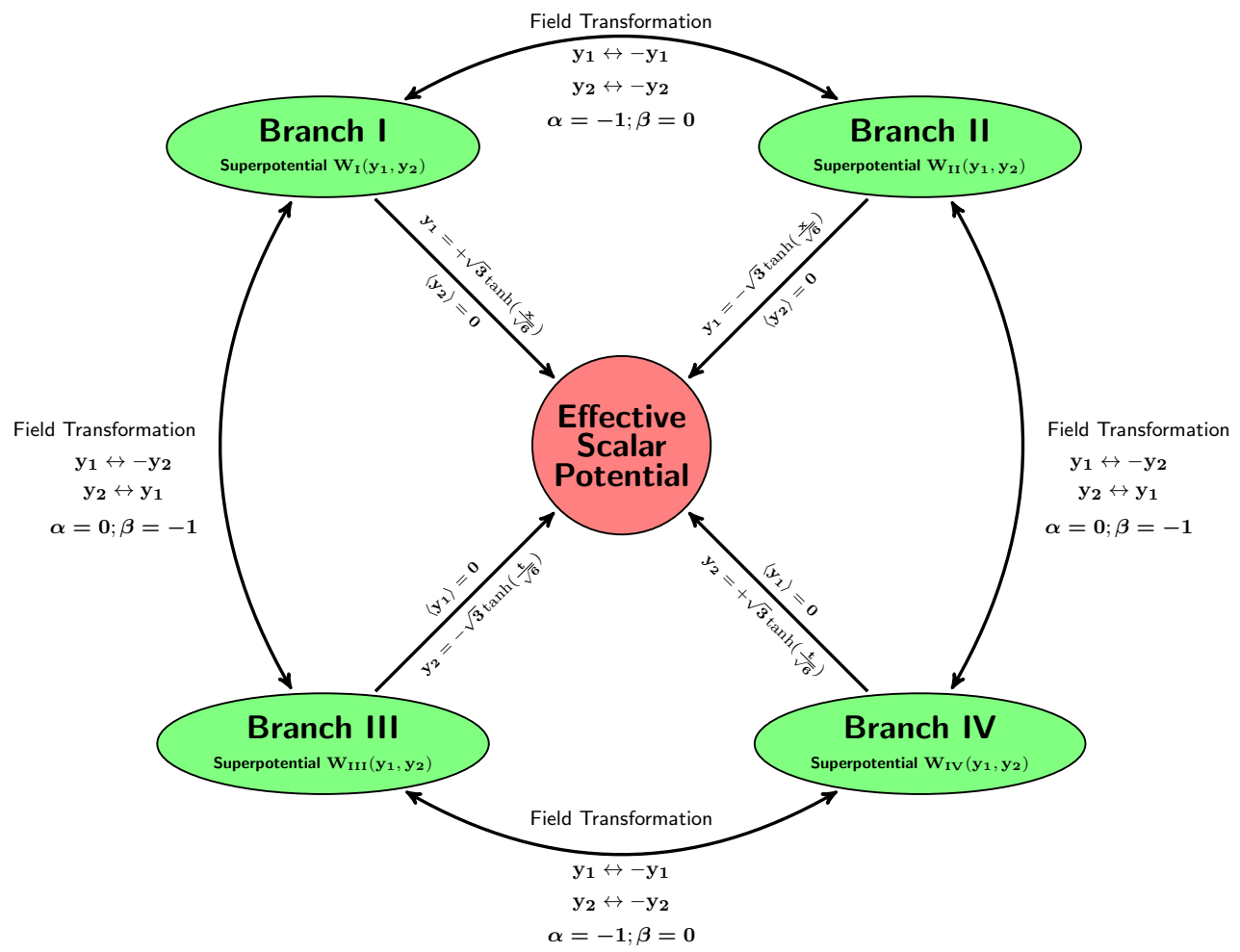

Fig. 9. Diagram illustrating the transformation laws between the superpotentials in different branches, together with the field fixings and canonical field redefinitions that yield the same Starobinsky-like effective scalar potential.

obtained from a general superpotential expression for Branch I of the form:

$$
\text { Branch I: } \quad W\left(y_{1}, y_{2}\right)=a y_{1}+b y_{1}^{2}+c y_{1}^{3}+d y_{2}+e y_{2} y_{1}+f y_{2} y_{1}^{2}+g\left(y_{1}, y_{2}\right),
$$

where $g\left(y_{1}, y_{2}\right)$ has the following properties: $g\left(y_{1}, 0\right)=0, \partial g / \partial y_{1}\left(y_{1}, 0\right)=0$ and $\partial g / \partial y_{2}\left(y_{1}, 0\right)=0$. The function $g$ may also include terms containing factors $y_{2}^{n}$, but these would not contribute to $V$, since we impose the condition $\left\langle y_{2}\right\rangle=0$. In order to avoid supersymmetry breaking close to the inflationary scale, we do not include a constant term in (128), nor in the general form of $W$ in the other branches.

Performing the transformation (127) with $\alpha=-1$ and $\beta=0$, we obtain the superpotential for Branch II. If, instead, we apply the transformation with $\alpha=0$ and $\beta=-1$ to the general expression for Branch I, we obtain the superpotential for Branch III. Finally, applying either the same transformation to Branch II or applying the previous transformation with $\alpha=-1$ and $\beta=0$ to Branch III, we obtain the superpotential of Branch IV.

Using the form 128 for the Branch I superpotential, we can derive $\hat{V}$ from 
(114) and match to a known solution from Ref. 82 ;

$$
\hat{V}=M^{2}\left|y_{1}\right|^{2}\left|1-y_{1} / \sqrt{3}\right|^{2},
$$

corresponding to the WZ model found in Ref. 20. Matching coefficients leads to four sets of solutions:

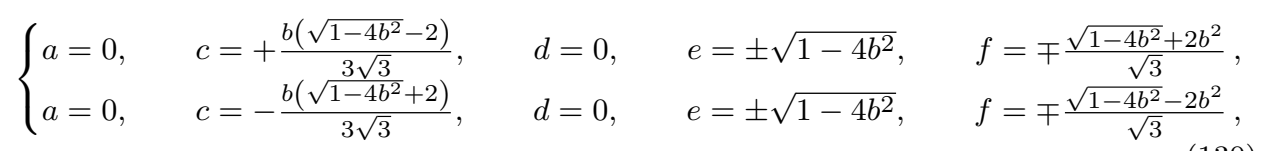

where all the coefficients are expressed in terms of a free parameter $b$. There are two additional solutions:

$$
\begin{aligned}
& b=-\frac{\sqrt{3} a}{2 a^{2}+3}, c=\frac{16 a^{6}+72 a^{4}+108 a^{2}+27}{36 a\left(2 a^{2}+3\right)^{2}}, d= \pm i a, \\
& e=\mp \frac{2 i \sqrt{3} a}{2 a^{2}+3}, f=\mp \frac{i\left(4 a^{2}\left(2 a^{2}+3\right)^{2}+27\right)}{12 a\left(2 a^{2}+3\right)^{2}},
\end{aligned}
$$

where now the coefficients are expressed in terms of a free parameter $a$. Eqs. 130 and (131) encompass all of the Branch I solutions corresponding to the Starobinsky inflationary potential with canonically-normalized kinetic terms.

The WZ model in Eq. 117] is a special case of the Branch I superpotential (128) with

$$
a, d, e=0 ; \quad b=\frac{1}{2} ; \quad c=-\frac{1}{3 \sqrt{3}} ; \quad f=\frac{1}{2 \sqrt{3}},
$$

as in Eq. 130 with $b=1 / 2$. This is just one specific example of the full set of Branch I solutions, all of which yield a Starobinsky potential. We could equally well have chosen a solution with $b=0$ giving

$$
a, b, c, d=0 ; \quad e=-1 ; \quad f=\frac{1}{\sqrt{3}},
$$

corresponding to the superpotential

$$
W=M y_{1} y_{2}\left(-1+\frac{y_{1}}{\sqrt{3}}\right) .
$$

We now consider this last solution and rotate it to Branch III as described in Fig. 9 i.e., we take $y_{1} \rightarrow-y_{2}$ and $y_{2} \rightarrow y_{1}$ giving

$$
W=M y_{1} y_{2}\left(1+\frac{y_{2}}{\sqrt{3}}\right) .
$$

Finally, we rotate back to the $(T, \phi)$ basis to obtain (dropping an irrelevant overall sign)

$$
W=\sqrt{3} M \phi\left(T-\frac{1}{2}\right)
$$


which is equivalent to another well-studied no-scale analogue of the $R+R^{2}$ theory 109 Several specific and equivalent examples of these avatars of no-scale Starobinsky models were discussed in Ref. 82 (see also Refs. 97, 104, 105, 106, 107, 108).

We conclude this Section by mentioning briefly an alternative, non-oscillatory no-scale supergravity model of inflation (NO-NO inflation) proposed recently ${ }^{109} \mathrm{In}$ this scenario there are no inflaton oscillations at the end of the inflationary era, and the expansion of the Universe is instead dominated by the kinetic energy density of the inflaton, a possibility called kination. When the Universe transitions from inflation to kination, it preheats instantly through a coupling to "Higgs" fields that annihilate rapidly and scatter producing matter particles. These later dominate the energy density, reheating the Universe to a temperature higher than during BBN. The NO-NO model makes predictions for CMB observables that are consistent with Planck 2018 data, produces gravitational waves with a density that is compatible with BBN, and produces via gravitino decay a density of supersymmetric cold dark matter that is also consistent with cosmological data.

\section{Stabilizing Moduli Fields in No-Scale Supergravity}

Up until this Section, we have implicitly assumed that all the real components of the complex fields that are not driving inflation have been stabilized, whereas the inflaton field has remained dynamical. In order not to spoil the inflationary dynamics, it is important to ensure that during inflation the other stabilized real fields remain fixed or at least strongly constrained. In this Section we do not attempt to review the problem of moduli stabilization, but instead we focus on certain specific examples of field stabilization mechanisms. However, the field stabilization mechanisms we discuss here do serve as existence proofs that lead to successful stabilization, whose origin we leave open.

In all the cases discussed here, we consider adding higher-order correction terms into the Kähler potential as first proposed in $\frac{110}{}$, and discussed more recently in 82 104:

$$
K=-3 \ln \left(T+T^{*}-\frac{|\phi|^{2}}{3}+\frac{\left(T+T^{*}\right)^{2 n}}{\Lambda_{T}^{2 n-2}}\right),
$$

where $n>1$ and $\Lambda_{T}$ is a mass scale which is smaller than the Planck scale $M_{P}$. For simplicity, hereafter we take the lowest possible value $n=2$, corresponding to a quartic term $\left(T+T^{*}\right)^{4}$.

The higher-order term in the Kähler potential (137) stabilizes the volume modulus $T$ in the real direction with $\operatorname{Re} T=0$. In order to stabilize the imaginary part of $T$, one may consider the following generalized Kähler potential form:

$$
K=-3 \ln \left(T+T^{*}-\frac{|\phi|^{2}}{3}+\frac{\left(T+T^{*}-2 c\right)^{4}+d\left(T-T^{*}\right)^{4}}{\Lambda_{T}^{2}}\right),
$$


where the term $d\left(T-T^{*}\right)^{4}$ stabilizes the potential in the imaginary direction of $T$ with $\operatorname{Im} T=0$, and we also introduced a field shift in the term $\left(T+T^{*}-2 c\right)^{4}$ that stabilizes the real part and leads to $\operatorname{Re} T=c$. In the case of the Wess-Zumino model (44), we saw how the Starobinsky potential can be obtained for the illustrative choice $c=1 / 2$. This modification of the Kähler potential fixes the volume modulus $T$ during inflation and generates the following masses

$$
m_{\operatorname{Re} T}^{2}=144 \frac{m_{3 / 2}^{2}}{\Lambda_{T}^{2}} ; \quad m_{\operatorname{Im} T}^{2}=144 d \frac{m_{3 / 2}^{2}}{\Lambda_{T}^{2}},
$$

which are hierarchically larger than the gravitino mass if $\Lambda_{T} \ll 1$. Since the quartic stabilization term in the Kähler potential (138) should be treated as an effective interaction, one must require that $\Lambda_{T}>m_{\operatorname{Re} T}, m_{\operatorname{Im} T}$, i.e., $\Lambda_{T}>$ $12 m_{3 / 2} / \Lambda_{T}, 12 \sqrt{d} m_{3 / 2} / \Lambda_{T}$.

In the case of the Wess-Zumino model that is expressed in the symmetric basis $\left(y_{1}, y_{2}\right)$ and given by Eq. (117), the inflationary dynamics are driven by the rolling inflaton field, $y_{1}$. During inflation, the effective potential is stabilized only in the imaginary direction of $y_{2}$ and we must prevent the real component of $y_{2}$ from acquiring a non-zero VEV, which would spoil the inflationary dynamics. To address this problem and stabilize the field $y_{2}$ in the real direction, one may introduce the following higher-order term in the symmetric Kähler potential 109):

$$
K=-3 \ln \left(1-\frac{\left|y_{1}\right|^{2}+\left|y_{2}\right|^{2}}{3}+\frac{\left|y_{2}\right|^{4}}{\Lambda^{2}}\right),
$$

where $\Lambda<1$, and the addition of the quartic stabilization term $\left|y_{2}\right|^{4} / \Lambda^{2}$ does not affect the inflationary potential $V\left(y_{1}\right)$ in the real $y_{1}$ direction.

Alternatively, if we consider models of inflation where inflation is driven by the volume modulus, $T$, e.g., the Cecotti superpotential (136) that also reproduces the Starobinsky model of inflation, one can modify the $\mathrm{SU}(2,1) / \mathrm{SU}(2) \times \mathrm{U}(1)$ Kähler potential $(102)$ with $\alpha=1$ by including a higher-order stabilization term for the matter-like field, $\phi$ :

$$
K=-3 \ln \left(T+T^{*}-\frac{|\phi|^{2}}{3}+\frac{|\phi|^{4}}{\Lambda_{\phi}^{2}}\right)
$$

where the quartic stabilization term $|\phi|^{4} / \Lambda_{\phi}^{2}$ stabilizes the effective potential in both the real and imaginary directions of $\phi$, and $\Lambda_{\phi}<1$.

The moduli stabilization mechanism discussed in this Section can be extended easily to multi-field models of inflation that are characterized by an $\mathrm{SU}(\mathrm{N}, 1) / \mathrm{SU}(\mathrm{N})$ $\times \mathrm{U}(1)$ coset space or more complicated coset structures, and the field stabilization of such models was discussed in ${ }^{93}$.

We mention in passing that quartic stabilization terms have also been considered ${ }^{111}$ in the context of the Polonyi mode ${ }^{221}$ discussed earlier. Adding a term

$$
\delta K=-\frac{|z|^{4}}{\Lambda_{z}^{2}}
$$


in either minimal or no-scale supergravity shifts the Planck-scale minimum. For example, the minimum found in minimal supergravity in Section 2.4 is shifted to

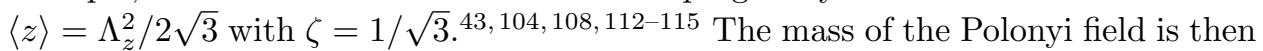
$m_{z}=\sqrt{12} m_{3 / 2} / \Lambda_{z}$, which is significantly heavier than in the minimal model when $\Lambda_{z} \ll 1$. Strong stabilization in this case can lead to a resolution of the cosmological problems associated with the Polonyi field that were discussed earlier. We discuss the strong stabilization of the Polonyi field in more detail in Section 11.2 .

We conclude this Section by mentioning an alternative stabilization mechanism, which does not require higher-order terms in the logarithm 101 103 In typical orbifold string compactifications with three moduli that are fixed by some unspecified mechanism at a high scale to be proportional, the Kähler potential takes the generic form 116

$$
K=-3 \ln \left(T+T^{*}-\frac{1}{3} \sum_{i}\left|\phi_{i}\right|^{2}\right)+\sum_{a} \frac{\left|\varphi_{a}\right|^{2}}{\left(T+T^{*}\right)^{n_{a}}},
$$

where $T$ is the volume modulus, the $\phi_{i}$ are untwisted matter fields, and the $\varphi_{a}$ are twisted matter fields with modular weights $n_{a}$. Coupling a twisted matter field with modular weight 3 to $T$ with a Cecotti-like superpotential (136), and

$$
K=-3 \ln \left(T+T^{*}\right)+\frac{|\varphi|^{2}}{\left(T+T^{*}\right)^{3}},
$$

the Starobinsky potential is recovered in the direction of the canonically-normalized $\operatorname{Re} T$, and there is a quadratic potential along the $\operatorname{Im} T$ direction. During inflation the effective scalar potential is proportional to the exponential factor $e^{K}$, or

$$
V \propto e^{|\varphi|^{2} /\left(T+T^{*}\right)^{3}} \simeq e^{|\varphi|^{2}},
$$

and the twisted field $\varphi$ is rapidly stabilized at the origin at the beginning of inflation. It is also worth noting that the inflationary dynamics of this model differ crucially from those with only untwisted fields. The real and imaginary parts of $T$ mix through their kinetic terms, leading to a coupling between curvature and isocurvature perturbations, and therefore an enhancement of the curvature modes at super-horizon scales. A correct discussion of their behavior during inflation requires a more sophisticated analysis than for the single-field models (see Ref. 103).

\section{No-Scale Inflation and Phenomenology}

We turn now to some phenomenological aspects of no-scale inflationary models. For this we work in the $(T, \phi)$ basis, and discuss the possibilities for supersymmetry breaking, the incorporation of matter and the generation of soft mass terms in the separate cases in which either $T$ or $\phi$ plays the role of the inflaton.

\section{1. $\phi$-Type Inflation}

As illustrated in (143), matter fields may be included in the Kähler potential as either untwisted or twisted fields depending whether their kinetic terms originate 
inside or outside the logarithm. We first consider the WZ model 444, where the inflaton is one of the untwisted matter fields, $\phi_{1}$. The superpotential for the matter fields can be written as

$$
\begin{aligned}
W=(T+c)^{\beta} W_{2}\left(\phi_{i}\right)+(T+c)^{\gamma} W_{3}\left(\phi_{i}\right) & \\
& +(T+c)^{\sigma} W_{2}\left(\varphi_{a}\right)+(T+c)^{\rho} W_{3}\left(\varphi_{a}\right)+\mu,
\end{aligned}
$$

where $c$ is an arbitrary constant, which we take to be $1 / 2$ for illustration, and $W_{2,3}$ denote bilinear and trilinear terms with modular weights that are in general nonzero. Here $\mu$ is a constant that contributes to supersymmetry breaking. Assuming that $T$ is properly stabilized with $\langle T\rangle=1 / 2$, the gravitino mass is simply

$$
m_{3 / 2}=\mu,
$$

and soft supersymmetry-breaking terms are easily calculated to be ${ }^{104}$

$$
\begin{array}{ll}
\text { Untwisted Matter Fields : } & m_{0}^{2}=\left(1-n_{a}\right)^{\frac{1}{2}} m_{3 / 2}, \\
m_{0}=0, & B_{0}=2\left(1-n_{a}-\frac{\sigma}{2}\right) m_{3 / 2}, \\
B_{0}=-\beta m_{3 / 2}, & A_{0}=3\left(1-n_{a}-\frac{\rho}{3}\right) m_{3 / 2} . \\
A_{0}=-\gamma m_{3 / 2}, &
\end{array}
$$

The form of Eq. 148 opens up various phenomenological possibilities.

If all matter fields are of the untwisted type, we see that there are no supersymmetry-breaking contributions to scalar masses, as expected in pure noscale supergravity. If in addition, the modular weights $\gamma$ and $\beta$ vanish, then $A_{0}=B_{0}=0$, and we recover the full set of no-scale boundary conditions ${ }^{[18}$ Radiative electroweak symmetry breaking ${ }^{119}$ can be accommodated if these boundary conditions are fixed at scales above the GUT scale $\frac{120}{125}$ In this case the parameter space is more restricted than in CMSSM-like models, since the ratio of the Higgs VEVs, $\tan \beta$, is determined by the Higgs minimization conditions and is no longer a free parameter. $\underline{40}$

If matter fields are of the twisted type, and the kinetic modular weights are 0 , we obtain universal soft scalar masses as in CMSSM-like models, which are determined by the gravitino mass ${ }^{[33}$ When the superpotential weights are equal $(\rho=\sigma)$, we obtain minimal mSUGRA-like boundary conditions, with $A_{0}=(3-\rho) m_{3 / 2}$ and $B_{0}=(2-\rho) m_{3 / 2}$, i.e., $B_{0}=A_{0}-m_{0} 33 \mid 40$ These mSUGRA-like models also yield a more restrictive parameter space. In the symmetric $\left(y_{1}, y_{2}\right)$ basis with no superpotential weights, we would find $\rho=\sigma=3$, in which case $A_{0}=0$ and $B_{0}=-m_{3 / 2}$. If, in addition, there are no tree-level sources for gaugino masses, the models would be equivalent to pure gravity mediation (PGM) with radiative electroweak symmetry breaking $\left[126 \mid 127\right.$ Finally, we note that if the weights $n_{a} \neq 0$, we have a source for non-universal scalar masses in the twisted sector.

One can also consider the effects of a Polonyi sector on $\phi$-type models, $\frac{104}{b u t}$ we defer a discussion of alternatives to the later Section on unified no-scale attractor 
models. We note that in Ref. 107 a term linear in $\phi$ is included, which plays the role of the Polonyi field, and Starobinsky-like inflation is possible so long as the gravitino mass $m_{3 / 2} \lesssim 1 \mathrm{PeV}$. The soft supersymmetry-breaking parameters for this model were derived in Ref. 128,

\subsection{T-Type Inflation}

As an example of $T$-type inflation, we consider the model first suggested in Ref. 99 , which is described by the superpotential (136), generalized by allowing the Kähler curvature to differ from $2 / 3$. We take the superpotential to be 90

$$
W_{I}=\sqrt{3 \alpha} M \phi\left(T-\frac{1}{2}\right)(2 T)^{\frac{3 \alpha-3}{2}} .
$$

In this case, supersymmetry cannot be broken by a constant term, as the minimum of the scalar potential is now found at

$$
T=\frac{1}{2}-\alpha \frac{\mu^{2}}{M^{2}}, \quad \phi_{1}=\sqrt{3 \alpha} \frac{\mu}{M}
$$

and the cosmological constant $V_{0} \simeq-3 \mu^{2}<0$. We can add in this case a Polonyi field ${ }^{22}$ with Kähler potential given by

$$
K \supset z z^{*}-\frac{\left(z z^{*}\right)^{2}}{\Lambda_{z}^{2}}
$$

where we include the strong stabilization of $z 43|104| 111|113| 115]$ We consider both possibilities of untwisted and twisted Polonyi fields. The superpotential is given by Eq. 14, but strong stabilization shifts the minimum to $z \simeq \Lambda_{z}^{2} / 3 \sqrt{12}\left(\Lambda_{z}^{2} / \sqrt{12}\right)$, for the untwisted (twisted) case, with the parameter $\zeta \simeq 1 / \sqrt{3}$ tuned to yield a vanishing cosmological constant $\underline{43 \| 112][13]}$ when $\alpha=1$. More generally, if we combine the Polonyi sector with the inflationary sector there is a shift in the supersymmetrybreaking minimum!

Untwisted Case :

$$
\begin{aligned}
& \langle T\rangle \simeq \frac{1}{2}+\left(\frac{2 \alpha-1}{3 \alpha}\right) \Delta^{2}, \\
& \langle\phi\rangle \simeq \Delta, \\
& \langle z\rangle \simeq \frac{\sqrt{\alpha}}{6 \sqrt{3}} \Lambda_{z}^{2}, \\
& \zeta \simeq \frac{1}{\sqrt{3 \alpha}}-\left(\frac{1+3 \alpha(\alpha-1)}{6 \sqrt{3} \alpha^{3 / 2}}\right) \Delta^{2}, \\
& \langle T\rangle \simeq \frac{1}{2}+\frac{2 \alpha}{3} \Delta^{2}, \\
& \langle\phi\rangle \simeq \sqrt{\alpha} \Delta, \\
& \langle z\rangle \simeq \frac{1}{2 \sqrt{3}} \Lambda_{z}^{2}, \\
& \zeta \simeq \frac{1}{\sqrt{3}}-\frac{\sqrt{3} \alpha^{2}}{6} \Delta^{2},
\end{aligned}
$$

where we define $\Delta \equiv \mu / M$ and assume that $\Delta, \Lambda_{z} \ll 1$. In this case, the form of the inflationary potential is unmodified from the Starobinsky form, save for the shift of the position of the minimum from $t_{0}=0$ to $t_{0}=\left(\frac{1}{\sqrt{\alpha}}-2 \sqrt{\alpha}\right) \sqrt{2 / 3} \Delta^{2}$ and
Twisted Case : 
$t_{0}=-2 \alpha^{3 / 2} \sqrt{2 / 3} \Delta^{2}$ for the untwisted and twisted cases respectively, where

$$
T=\frac{1}{2}\left(e^{-\sqrt{\frac{2}{3 \alpha}} t}+i \sqrt{\frac{2}{3}} \sigma\right),
$$

and $t$ and $\sigma$ denote the canonically-normalized real and imaginary part of $T$, respectively, and we associate $t$ with the inflaton. The supersymmetry-breaking scale given by the gravitino mass is $m_{3 / 2}=\mu / \sqrt{3 \alpha}(\mu / \sqrt{3})$ for untwisted (twisted) $z$. The mass of the canonically-normalized Polonyi field is $m_{z}^{2}=36 m_{3 / 2}^{2} / \Lambda_{z}^{2}\left(12 m_{3 / 2}^{2} / \Lambda_{z}^{2}\right)$ and, as discussed earlier, is hierarchically larger than $m_{3 / 2}$, thereby alleviating the cosmological Polonyi problem 43115

When combined with the superpotential (146) that includes matter fields, we obtain the following universal soft supersymmetry-breaking parameters 104,115 when the Polonyi field is untwisted:

$$
\begin{array}{ll}
\text { Untwisted Matter Fields : } & \text { Twisted Matter Fields : } \\
m_{0}^{2}=(\alpha-1) m_{3 / 2}^{2}, & m_{0}^{2}=m_{3 / 2}^{2}, \\
B_{0}=-m_{3 / 2}, & B_{0}=-m_{3 / 2}, \\
A_{0}=0, & A_{0}=0 .
\end{array}
$$

We note that in this case there is no dependence on the modular weights. As one can see, the only dependence of the soft supersymmetry-breaking terms on the curvature parameter $\alpha$ that appears in the soft scalar masses for untwisted matter fields. When $\alpha=1$, we have vanishing input scalar masses, as is typical in no-scale models. When $\alpha=2$, we obtain $m_{0}=m_{3 / 2}, B_{0}=-m_{3 / 2}$, and $A_{0}=0$, which is the same as the pattern of soft terms when matter fields are twisted. These are of the mSUGRA type when the gaugino masses are of order $m_{3 / 2}$ and of the PGM type if gaugino masses are generated through anomalies.

When the Polonyi field is twisted we find

$$
\begin{array}{ll}
\text { Untwisted Matter Fields : } & \text { Twisted Matter Fields : } \\
m_{0}^{2}=\alpha m_{3 / 2}^{2}, & m_{0}^{2}=m_{3 / 2}^{2}, \\
B_{0}=-m_{3 / 2}, & B_{0}=-m_{3 / 2}, \\
A_{0}=0, & A_{0}=0 .
\end{array}
$$

The soft terms for twisted matter fields are unchanged from Eq. 154 and, as before, only the untwisted matter fields have a dependence on $\alpha$. In this case, because $m_{0}=\sqrt{\alpha} m_{3 / 2}$, the only restriction we have is $\alpha>0$.

\subsection{Unified no-scale attractors}

As an alternative to breaking supersymmetry with a Polonyi field, we can use the two fields in the inflationary sector to break supersymmetry, leaving a small residual vacuum energy that could be identified as dark energy $94[115 \mid 129$ Such models have been called unified no-scale attractors. We consider a simple case with a single 
modulus, $T$, and a single matter field, $\phi$, that we associate with the inflaton, so that $\xi=2 T-\phi^{2} / 3$. We specialize to the case $\alpha=1$, and refer the interested reader to Ref. 94 for a generalization. Such models can also be formulated with a twisted inflaton, $\varphi$, see Ref. 115 .

In this simple set-up, the inflationary superpotential (103) reduces to (44), and the de Sitter superpotential 97) reduces to

$$
W_{d S}=\lambda_{1} M^{3}-\lambda_{2} M^{3}\left(2 T-\frac{\phi^{2}}{3}\right)^{3},
$$

as $n=0$ and $\bar{n}=3$, and we have scaled the constants in $W_{d S}$ with the cube of the inflaton mass, $M$. The superpotential then becomes

$$
W=W_{I}+W_{d S}=M\left(\frac{\phi^{2}}{2}-\frac{\phi^{3}}{3 \sqrt{3}}\right)+\lambda_{1} M^{3}-\lambda_{2} M^{3}\left(2 T-\frac{\phi^{2}}{3}\right)^{3} .
$$

The unified Wess-Zumino model 157) with the fields fixed at $\langle T\rangle=\frac{1}{2}$ and $\langle\operatorname{Im} \phi\rangle=$ 0 then yields the following scalar potential:

$$
V=12 \lambda_{1} \lambda_{2} M^{6}+12 \lambda_{2} M^{4}\left(\frac{\phi^{2}}{2}-\frac{\phi^{3}}{3 \sqrt{3}}\right)+3 M^{2}\left(\frac{\phi}{\sqrt{3}+\phi}\right)^{2},
$$

which becomes

$V=12 \lambda_{1} \lambda_{2} M^{6}+6 \lambda_{2} M^{4} \tanh ^{2}\left(\frac{x}{\sqrt{6}}\right)\left(3-2 \tanh \left(\frac{x}{\sqrt{6}}\right)\right)+\frac{3}{4} M^{2}\left(1-e^{-\sqrt{\frac{2}{3}} x}\right)^{2}$

after the canonical field redefinition 120 .

The first term in 159 is a cosmological constant with the value $\Lambda=12 \lambda_{1} \lambda_{2} M^{6}$. The vacuum energy density will be modified by contributions from phase transitions occurring after inflation, which are negative in general. If $\lambda_{1,2} \sim \mathcal{O}(1)$, these contributions should be of order $M^{6} \sim 10^{-30}$ in order to (almost) cancel the first term in 159 and yield a net cosmological constant of order $10^{-120}$ today, in natural units. We note in this connection that the GUT phase transition in a flipped $\mathrm{SU}(5) \times \mathrm{U}(1)$ GUT model is expected to occur after inflation ${ }^{130}$ and to contribute $\Delta V \sim-M_{\text {susy }}^{2} M_{\text {GUT }}^{2} \sim-\left(\lambda_{1}-\lambda_{2}\right)^{2} M^{6} M_{\text {GUT }}^{2}$, suggesting that a hierarchy $\lambda_{1} / \lambda_{2}$ or $\lambda_{2} / \lambda_{1} \sim\left(M_{\mathrm{GUT}} / M_{P}\right)^{2}$ should be preferred (see below).

The second term in 159 perturbs the inflaton potential given by the third term in (159), but is unimportant for the inflationary dynamics, since it has a prefactor of $M^{4}$, whereas the inflationary potential is scaled by $M^{2}$. This term adds a relatively small amount, $6 \lambda_{2} M^{4}$, to the Starobinsky plateau height $(3 / 4) M^{2}$ at large $x$.

At the end of inflation, supersymmetry is broken through an $F$-term for $T$, which is given by $\left[\begin{array}{ll}129 \\ 129\end{array}\right.$

$$
\sum_{i=1}^{2}\left|F_{i}\right|^{2}=F_{T}^{2} \simeq\left(\lambda_{1}+\lambda_{2}\right)^{2} M^{6}
$$


where $F_{T}$ is derived using Eq. 12, and the gravitino mass is given by

$$
m_{3 / 2}=e^{G / 2}=e^{K / 2} W=\left(\lambda_{1}-\lambda_{2}\right) \frac{M^{3}}{M_{P}^{2}} .
$$

To obtain a gravitino mass $m_{3 / 2} \simeq \mathcal{O}(1) \mathrm{TeV}$, we choose $\lambda_{2} \ll \lambda_{1}$, in which case

$$
m_{3 / 2}=\left(\lambda_{1}-\lambda_{2}\right) \frac{M^{3}}{M_{P}^{2}} \simeq \lambda_{1} \frac{M^{3}}{M_{P}^{2}}
$$

and $F_{T} \simeq m_{3 / 2}$. By scaling $W_{d S}$ with $M^{3}$, we are able to obtain a TeV mass scale for supersymmetry breaking without fine-tuning. Furthermore, we relate the supersymmetry-breaking scale to the inflation scale $M$ (see also Refs. 108, 114).

It is now relatively straightforward to add Standard Model fields to the model, and the full superpotential can be written as

$$
W=W_{I}+W_{d S}+W_{S M},
$$

where $W_{S M}$ can be written in a form similar to Eq. 146). For $\alpha \neq 1$ and recalling the definitions $n_{ \pm}=\frac{3}{2}(\alpha \pm \sqrt{\alpha})$ (86, we should multiply the expression in 146 by a factor of $\sqrt{\alpha} \xi^{n_{-}}$, where $\xi=2 T-\frac{\phi^{2}}{3}(\xi=2 T)$, assuming here that the inflaton is untwisted (twisted). Similar results can be obtained using instead $\sqrt{\alpha} \xi^{n_{+}}$. The resulting soft terms are very similar to those in Eq. [148,!115]

$$
\begin{array}{ll}
\text { Untwisted Matter Fields : } & \text { Twisted Matter Fields : } \\
m_{0}^{2}=(\alpha-1) m_{3 / 2}^{2}, & m_{0}^{2}=\frac{\left(\alpha-n_{a}\right)}{\alpha} m_{3 / 2}^{2}, \\
B_{0}=(2 \sqrt{\alpha}-2-\beta) m_{3 / 2}, & B_{0}=\left(2 \sqrt{\alpha}-2 n_{a}-\sigma\right) m_{3 / 2}, \\
A_{0}=(3 \sqrt{\alpha}-3-\gamma) m_{3 / 2}, & A_{0}=\left(3 \sqrt{\alpha}-3 n_{a}-\rho\right) m_{3 / 2} .
\end{array}
$$

For $\alpha=1$, these results reduce to those in Eq. 148)!104

In the superpotentials in Eqs. 157) and (163), the volume modulus plays the role of the Polonyi field and is responsible for supersymmetry breaking. As a result, it is subject to similar cosmological constraints as the Polonyi field to avoid excessive entropy production or an excessive dark matter abundance produced by modulus decay. However the strong stabilization of $T$ helps resolve these issues in a similar way to a strongly-stabilized Polonyi field $\stackrel{43}{4}$

\subsection{Constraints on the Stabilization Parameter $\Lambda_{T}$}

As noted in Section 2.4 cosmological problems 41 arise when the modulus field value after inflation is displaced from its potential minimum. In the absence of strong stabilization, the displacement is $\mathcal{O}\left(M_{P}\right)$. Stabilization reduces the amplitude to a maximum displacement of $\Lambda_{T} / 4 \sqrt{3}$. Thus, after the period of exponential expansion, both the inflaton, $\phi$, and the modulus, $T$, undergo scalar field oscillations until they decay. These oscillations begin when the Hubble parameter, $H$, is roughly $\frac{2}{3} m_{\phi, t}$, and $H=(\rho / 3)^{1 / 2}$ may be determined by the energy density, $\rho$, stored in either 
inflaton or modulus oscillations, or the radiation energy produced by inflaton decays. In this model, $m_{\phi}=M$, and the mass of the canonically-normalized modulus is $m_{t}$, given by $1 / \sqrt{3 \alpha}$ times the value in Eq. 139 . We parametrize the inflaton decay rate here as

$$
\Gamma_{\phi}=d_{\phi}^{2} \frac{M^{3}}{M_{P}^{2}},
$$

where $d_{\phi}$ is a model dependent gravitational-strength coupling.

We can distinguish several possible histories for the fields $\phi$ and $T$, depending on the various masses and the inflaton decay coupling, $d_{\phi}{ }^{[15]}$ (See also Ref. 131 for a review of cosmological moduli.) Here we focus on one scenario in particular, where $T$ oscillations begin before inflaton decay. We refer to this as Scenario I (as opposed to Scenario II when $T$ oscillations begin after inflaton decay). Furthermore, we can also distinguish if $T$ decays before the inflaton ( $\mathrm{I}$ a), after the inflaton but before $T$ oscillations dominate the energy density ( $\mathrm{I}$ b), and when they do dominate the energy density (I c). These regions are shown in the $\left(m_{3 / 2}, \Lambda_{T}\right)$ plane in Fig. 10 for $d_{\phi}=10^{-3}$. The derivations of the boundaries of these subregions can be found in Ref. [115.

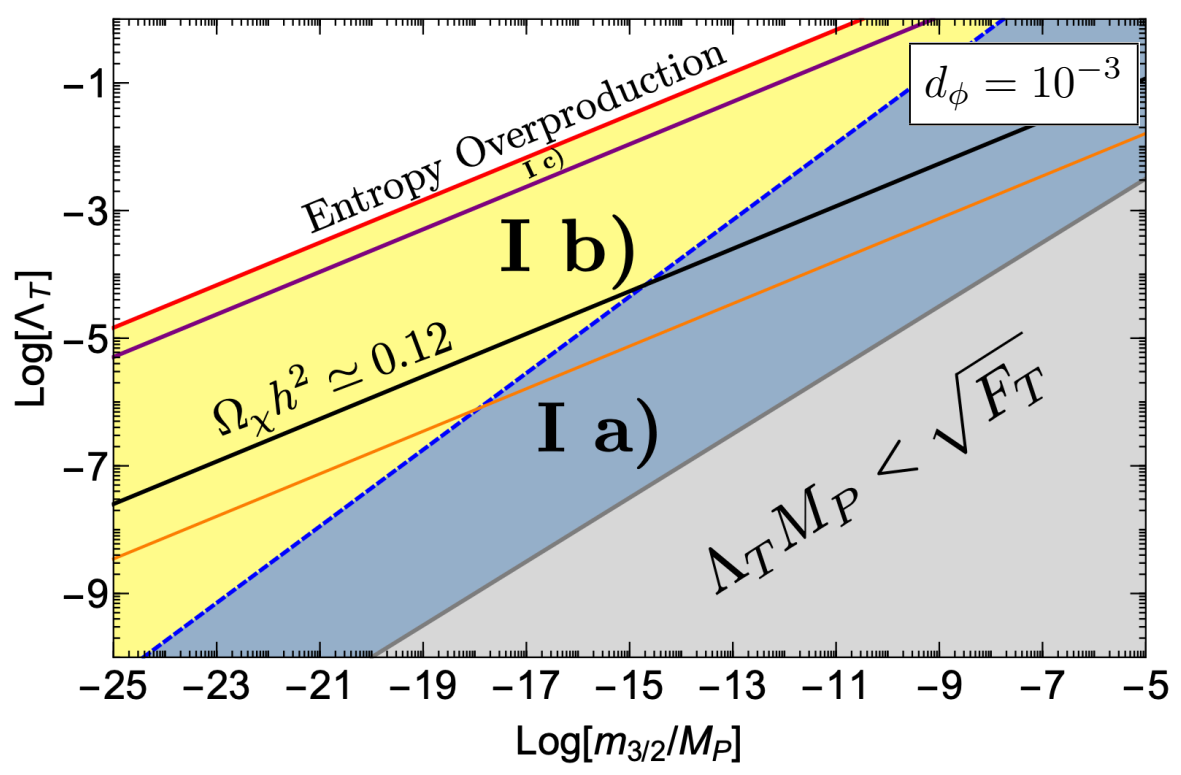

Fig. 10. Plot of the constraints on the modulus stabilization parameter, $\Lambda_{T}$, as a function of the gravitino mass, $m_{3 / 2}$, for models with $\alpha=1$ and $d_{\phi}=10^{-3}$. The regions shaded yellow and blue correspond, respectively, to the Scenarios $I b, c)$, and $I$ a) described in the text, whereas the grey regions are excluded by the effective interaction condition (168). Regions between this and the dark matter density constraint (solid black line) are allowed by all the constraints.

As long as $T$ decays while the Universe is either dominated by the inflaton 
oscillations ( $\mathrm{I}$ a) or radiation produced by inflaton decays ( $\mathrm{I}$ b), the entropy produced by $T$ decays is negligible. However, if $T$ decays late, when it dominates the total energy budget, a significant amount of entropy production is possible. The amount of allowed entropy production is model-dependent, and in some cases necessary. For example, in the case of Affleck-Dine baryogenesis, $\frac{132}{1}$ the initial baryon-to-entropy ratio may be large, and some dilution due to late inflaton decays ${ }^{133}$ and/or moduli decays $\$ 134$ is welcome. It is possible to derive an upper limit on $\Lambda_{T}{ }^{115}$

$$
\Lambda_{T} \lesssim 2 \alpha^{1 / 6} \sqrt{3}\left(\frac{256}{\pi}\right)^{1 / 9} d_{\phi}^{-2 / 9} \Delta^{2 / 9}\left(\frac{m_{3 / 2}}{M}\right)^{1 / 3}
$$

where $\Delta$ is the maximum allowed dilution factor. This limit is shown in Fig. 10 as the boundary above region I c, assuming $\Delta<100$.

A bound stronger than that due to entropy production can be derived from the production of cold dark matter, since $T$ decays into pairs of gravitinos that subsequently decay into LSPs could result in an overabundance of cold dark matter. We can derive the following upper limit on $\Lambda_{T} \underline{\underline{115}}$

$$
\Lambda_{T} \lesssim 2 \times 10^{-3} \alpha^{-1 / 6} d_{\phi}^{-1 / 3} \frac{m_{3 / 2}^{1 / 3} M_{P}^{1 / 6}}{M^{1 / 2}}\left(\frac{m_{\chi}}{100 \mathrm{GeV}}\right)^{-1 / 3} .
$$

This limit is seen as the black line cutting through regions I a) and I b) in Fig. 10 as labelled. Below the orange line (running parallel and below the black line), thermal production of gravitinos equals that from $T$ decays.

Finally, we note that there is a lower limit on $\Lambda_{T}$ coming from the postulated form of the stabilization terms in the Kähler potential. Since these should be treated as effective interactions obtained by integrating out fields with masses $\mathcal{O}\left(\Lambda_{T}\right)$, we require $\Lambda_{T}>\sqrt{F_{T}}, 108[111] 114$ and using $(160)$ we find that the limit

$$
\Lambda_{T}>\alpha^{-1 / 4}\left(\frac{m_{3 / 2}}{M_{P}}\right)^{1 / 2}
$$

is imposed by the effective interaction assumption. The area violating this limit is shaded grey in Fig. 10 .

\subsection{Reheating}

No model of inflation is complete without a discussion of reheating. On the one hand, it is essential for making contact with physics at the $\mathrm{TeV}$ scale and the Standard Model and, on the other hand, the amount of reheating affects the estimate of $N_{*}$ and hence, in particular, the amount of scalar tilt predicted within any given model. As in the previous Subsections, we treat the cases of $\phi$-type and $T$-type inflation separately.

\subsubsection{Reheating in $\phi$-type models}

Reheating is determined by the couplings of the inflaton to SM fields, which depend on the forms of $K, W$, and their derivatives. If we assume that all matter fields 
$\{\phi, \varphi\}$ have vanishing VEVs at the end of inflation, we find that

$$
\left\langle W^{i}\right\rangle=\left\langle W^{a}\right\rangle=0, \quad\left\langle K^{i}\right\rangle=\left\langle K^{a}\right\rangle=0,
$$

where $i, a$ are indices for untwisted and twisted fields, respectively, and hence in terms of the Kähler function,

$$
G^{i}=G^{a}=0 .
$$

In contrast, $\left\langle G_{T}\right\rangle=-3$, and we have $\langle W\rangle=\mu$.

If, as we have assumed until now, there are no direct superpotential couplings between $\phi$ and other fields, so that $W_{1 I}=0$, where the index 1 corresponds to the inflaton, and the index $I=\{T, i, a\}$ represents the volume modulus $T$, untwisted matter fields, and twisted matter fields, then there are no decay channels directly to matter scalars 104 Note, however, that by adding a superpotential term such as $\zeta \phi_{1}(T-1 / 2)^{2}$, which does not affect the dynamics of inflation, we obtain a decay to moduli with rate

$$
\Gamma\left(\phi_{1} \rightarrow \delta T \delta T\right)=M \frac{|\zeta|^{2}}{72 \pi},
$$

where $\delta T=\sqrt{3}(T-1 / 2)$ is the canonically-normalized modulus fluctuation. Decays to a pair of gravitinos would proceed at the same rate.104 However, if these are dominant they could lead to overproduction of the LSP that, if it is stable, would exceed the bounds on the cold dark matter density.

Alternatively, a direct coupling between $\phi_{1}$ and the matter sector may be allowed. For example, this field may be associated with a heavy singlet sneutrino $\sqrt[123[136]{ } \text { In such a case, one can consider the addition of a Yukawa-like term }$

$$
\Delta W=y_{\nu} H_{u} L \phi_{1}
$$

to the Standard Model superpotential, where $y_{\nu}$ denotes the Yukawa coupling. Such a coupling leads to a scalar mass matrix characteristic of seesaw models:

$$
\left(\begin{array}{ll}
\bar{\phi}^{1} & \overline{\tilde{\nu}}
\end{array}\right)\left(\begin{array}{cc}
M^{2}+\tilde{m}^{2} & -M \tilde{m} \\
-M \tilde{m} & \tilde{m}^{2}+\kappa \mu^{2}
\end{array}\right)\left(\begin{array}{c}
\phi_{1} \\
\tilde{\nu}
\end{array}\right)
$$

where $\tilde{m} \equiv y_{\nu}\left\langle H_{u}\right\rangle=y_{\nu} v \sin \beta, \kappa=\left(1-n_{\nu}\right)$ for a twisted neutrino, $\kappa=0$ for an untwisted neutrino, and $v \simeq 174 \mathrm{GeV}$ is the SM Higgs VEV. Even in the presence of direct couplings, we can consider $\phi_{1}$ to be the inflaton mass eigenstate, up to corrections of order $\mu / M, v / M \ll 1$.

In this case, we have a coupling $-M y_{\nu} H^{*}{ }_{u} \tilde{L}^{*} \phi_{1}$, and the inflaton decay width is given by

$$
\Gamma\left(\phi_{1} \rightarrow H_{u}^{0} \tilde{\nu}, H_{u}^{+} \tilde{f}_{L}\right)=M \frac{\left|y_{\nu}\right|^{2}}{16 \pi},
$$

where we have neglected the masses of the final-state particles. This decay rate would be fast if $\left|y_{\nu}\right| \sim \mathcal{O}(1)$. However, in order to avoid problems associated with 
gravitino overproduction during reheating, we must set a bound on the Yukawa coupling associated with the inflaton! 123

$$
y_{\nu} \lesssim 10^{-5}
$$

leading to a corresponding constraint on the reheating temperature, $T_{\mathrm{RH}}$, whose derivation we discuss below.

As in the scalar case, all couplings to matter fermions vanish for a $\phi_{1}$ independent matter superpotential. However, the decay into a fermion and a higgsino is possible if we identify $\phi_{1}$ with a singlet neutrino, with superpotential 172 . In this case, the rate is given by

$$
\Gamma\left(\phi_{1} \rightarrow \tilde{H}_{u}^{0} \nu, \tilde{H}_{u}^{+} f_{L}\right)=M \frac{\left|y_{\nu}\right|^{2}}{16 \pi},
$$

i.e., equal to the rate of decay into scalars.

If we assume instantaneous inflaton decay and thermalization of decay products, we can easily relate the reheating temperature to the inflaton decay rate $46 \mid 137$ After the period of exponential expansion, the inflaton begins to oscillate about its minimum when the Hubble parameter, $H=\frac{2}{3} M$. If we define $R_{\phi}$ as the scale factor when inflaton oscillations begin, we can write the energy density and Hubble parameter as

$$
\begin{aligned}
\rho_{\phi} & \simeq \frac{4}{3} M^{2} M_{P}^{2}\left(\frac{R_{\phi}}{R}\right)^{3}, \\
H & \simeq \frac{2}{3} M\left(\frac{R_{\phi}}{R}\right)^{3 / 2} .
\end{aligned}
$$

We can further define the time of decay by $\Gamma_{\phi} t=1$, or $H=\frac{2}{3} \Gamma_{\phi}$. Then, if all of the energy density in oscillations is converted to radiation we have

$$
T_{\mathrm{RH}}=\left(\frac{40}{g_{\mathrm{RH}} \pi^{2}}\right)^{1 / 4}\left(\Gamma_{\phi} M_{P}\right)^{1 / 2},
$$

where $g_{\mathrm{RH}} \equiv g\left(T_{\mathrm{RH}}\right)$ is the number of effective degrees of freedom in the thermal bath at $T_{\mathrm{RH}}$. For $M=3 \times 10^{13} \mathrm{GeV}$ and $g_{\mathrm{RH}}=915 / 4$, we have

$$
T_{\mathrm{RH}}=6.2 \times 10^{14} y_{\nu} \mathrm{GeV} .
$$

The abundance of gravitinos produced thermally can be expressed as 64

$$
\frac{n_{3 / 2}}{s} \simeq 2.6 \times 10^{-4}\left(1+0.56 \frac{m_{1 / 2}^{2}}{m_{3 / 2}^{2}}\right)\left(\frac{\Gamma_{\phi}}{M_{P}}\right)^{1 / 2},
$$

and requiring that the abundance of dark matter produced by gravitino decay is $\Omega_{\chi} h^{2}<0.12$ we have

$$
\frac{n_{3 / 2}}{s}<4.4 \times 10^{-12}\left(\frac{100 \mathrm{GeV}}{m_{\chi}}\right)
$$


leading to the limit 175 imposed by the production of gravitinos from the thermal bath $56[57 \mid 60 \quad 65$

If the gauge kinetic term, $f_{\alpha \beta}$, is non-trivial and depends on $\phi_{1}, f_{\alpha \beta}=f\left(\phi_{1}\right) \delta_{\alpha \beta}$, a decay channel for inflaton decay into gauge fields and gauginos is also possible $75\left[104 \sqrt{135}\right.$ So long as supersymmetry is not broken by the inflaton $\left(F_{\phi_{1}}=0\right)$, this term does not contribute to gaugino masses, which are proportional to the $F$ term and derivatives of the gauge kinetic function as seen in Eq. 200. The decay widths to canonically-normalized gauge boson pairs and gauginos are ${ }^{135}$

$$
\Gamma\left(\phi_{1} \rightarrow g g\right)=\Gamma\left(\phi_{1} \rightarrow \tilde{g} \tilde{g}\right)=\frac{3 d_{g, 1}^{2}}{32 \pi}\left(\frac{N_{G}}{12}\right) \frac{M^{3}}{M_{P}^{2}},
$$

where $N_{G}=12$ in the Standard Model, and $d_{g, 1}$ is given by

$$
d_{g, 1} \equiv\langle\operatorname{Re} f\rangle^{-1}\left|\left\langle\frac{\partial f}{\partial \phi_{1}}\right\rangle\right|
$$

This leads to a reheating temperature of

$$
T_{\mathrm{RH}}=6.7 \times 10^{9} d_{g, 1} \mathrm{GeV} .
$$

Thus the cold dark matter density is roughly saturated by thermally-produced gravitinos during reheating when the coupling $d_{g, 1} \sim 1$.

\subsubsection{Reheating in T-type models}

We again assume the absence of VEVs for matter fields and that the conditions 169 hold. In contrast to the case of a matter-like inflaton, the $T$ field couples to the matter sector through the supergravity Lagrangian. Two-body decays are possible with a rate given by 104

$$
\Gamma\left(T \rightarrow \Phi_{I} \bar{\Phi}^{J}\right)=\left(n_{I}+n_{L}-3\right)^{2} \frac{\left|W^{I L} \bar{W}_{L J}\right|^{2}}{48 \pi M M_{P}^{2}},
$$

where the $n_{I, L}$ are modular weights, and a sum over the repeated index $L$ is implied. This rate is weak-scale suppressed in the case of MSSM scalars. For example, the rate for decay to two Higgs bosons is

$$
\Gamma\left(T \rightarrow H_{u, d} \bar{H}^{u, d}\right)=\left(2 n_{H}-3\right)^{2} \frac{\left|\mu_{H}\right|^{4}}{24 \pi M M_{P}^{2}},
$$

where $\mu_{H}$ denotes the bilinear Higgs coupling in the MSSM. This two-body rate would lead to an extremely low reheating temperature: for an inflaton mass $M \sim$ $10^{-5} M_{P}$, and $\mu_{H} \sim 1 \mathrm{TeV}, T_{\mathrm{RH}} \sim 10^{-1} \mathrm{eV}$. We note, however, that in the case of high-scale supersymmetry 138 where all superpartners except the gravitino are heavier than the inflaton $65 \sqrt[608]{1144}|139| \frac{142}{\mid 14}$ the decay to Higgs pairs may be the dominant decay channel $108|114| 141 \mid 143$. 
Three-body decays to light scalars are possible and the widths are given by

$$
\begin{aligned}
& \Gamma\left(T \rightarrow \Phi_{I} \bar{\Phi}^{J} \bar{\Phi}^{K}\right)=\left(n_{I}+n_{L}-3\right)^{2} \frac{\left|W^{I L} \bar{W}_{L J K}\right|^{2} M}{12(8 \pi)^{3} M_{P}^{2}}, \\
& \Gamma\left(T \rightarrow \Phi_{I} \Phi_{J} \bar{\Phi}^{K}\right)=\left(n_{I}+n_{J}+n_{L}-3\right)^{2} \frac{\left|W^{I J L} \bar{W}_{L K}\right|^{2} M}{12(8 \pi)^{3} M_{P}^{2}} .
\end{aligned}
$$

For example, the decay to the neutral $d$-type Higgs and the left and right stops has the rate

$$
\Gamma\left(T \rightarrow \bar{H}_{d}^{0} \overline{\tilde{t}}_{R} \tilde{t}_{L}, H_{d}^{0} \tilde{t}_{R} \overline{\tilde{t}}_{L}\right)=\left(\left(2 n_{H}-3\right)^{2}+\left(2 n_{t}+n_{H}-3\right)^{2}\right) \frac{\left|\mu_{H} y_{t}\right|^{2} M}{4(8 \pi)^{3} M_{P}^{2}},
$$

where $y_{t}$ denotes the top Yukawa coupling. If this were dominant, the corresponding reheating temperature would also be low, in the $\mathrm{MeV}$ range.

However, we find that the rates for four-body decays are the largest, despite being phase-space suppressed. The decay width

$$
\Gamma\left(T \rightarrow \Phi_{I} \Phi_{J} \bar{\Phi}^{K} \bar{\Phi}^{M}\right)=\left(n_{I}+n_{J}+n_{L}-3\right)^{2} \frac{\left|W^{I J L} \bar{W}_{L K M}\right|^{2} M^{3}}{72(8 \pi)^{5} M_{P}^{2}},
$$

where we have disregarded the bilinear couplings, implies the following decay rate to four stops

$$
\Gamma\left(T \rightarrow \tilde{t}_{R} \tilde{t}_{L} \overline{\tilde{t}}_{R} \overline{\tilde{t}}_{L}\right)=\left(2 n_{t}+n_{H}-3\right)^{2} \frac{\left|y_{t}\right|^{4} M^{3}}{8(8 \pi)^{5} M_{P}^{2}},
$$

which corresponds to

$$
T_{\mathrm{RH}}=\left|2 n_{t}+n_{H}-3\right|\left(4.3 \times 10^{6} \mathrm{GeV}\right)\left|y_{t}\right|^{2} .
$$

Thus, as long as the matter fields do not reside in the untwisted sector (for which $n_{i}=1$ and the rate vanishes), we can obtain a reheating temperature well above that required for successful Big Bang Nucleosynthesis (BBN).

There are also decays to matter fermions. The rates for two-body decays to matter fermions take the form

$$
\Gamma\left(T \rightarrow \bar{\chi}_{I} \chi_{J}\right)=\left(n_{I}+n_{J}-3\right)^{2} \frac{\left|W^{I J}\right|^{2} M}{192 \pi M_{P}^{2}},
$$

which are $1 / 4$ of the rate for three-body decays into scalars.

The dominant rates are for three-body decays involving two fermions and one matter scalar, which are

$$
\Gamma\left(T \rightarrow \bar{\chi}_{I} \chi_{J} \Phi_{K}\right)=\left(n_{I}+n_{J}+n_{K}-3\right)^{2} \frac{\left|W^{I J K}\right|^{2} M^{3}}{36(8 \pi)^{3} M_{P}^{2}} .
$$

These are non-vanishing in the MSSM so long the fields are twisted with weights $n_{i} \neq 1$. In particular, in the case of the top quark one has the decay rate

$$
\Gamma\left(T \rightarrow H_{u}^{0} t_{L} \bar{t}_{R}, \tilde{t}_{L} \tilde{H}_{u}^{0} \bar{t}_{R}, \overline{\tilde{t}}_{R} t_{L} \tilde{H}_{u}^{0}\right)=\left(2 n_{t}+n_{H}-3\right)^{2} \frac{\left|y_{t}\right|^{2} M^{3}}{12(8 \pi)^{3} M_{P}^{2}},
$$


which is somewhat larger than the four-scalar decay rate 192 , because of the threebody phase-space factor. This decay rate would lead to a reheating temperature

$$
T_{\mathrm{RH}}=\left(8.9 \times 10^{7} \mathrm{GeV}\right)\left|y_{t}\left(2 n_{t}+n_{H}-3\right)\right| .
$$

Finally, if the gauge kinetic function depends on $T$, decays to gauge bosons and gauginos are also possible. The decay width to the canonically-normalized gauge boson pairs is readily evaluated, resulting in

$$
\Gamma(T \rightarrow g g)=\frac{d_{g, T}^{2}}{32 \pi}\left(\frac{N_{G}}{12}\right) \frac{M^{3}}{M_{P}^{2}},
$$

where

$$
d_{g, T} \equiv\langle\operatorname{Re} f\rangle^{-1}\left|\left\langle\frac{\partial f}{\partial T}\right\rangle\right|
$$

and the corresponding reheating temperature is

$$
T_{\mathrm{RH}}=\left(3.8 \times 10^{9} \mathrm{GeV}\right) d_{g, T} .
$$

The coefficient $d_{g, T}$ might well be $\mathcal{O}(1)$, e.g., for a gauge kinetic function linear in $T$ with $\mathcal{O}(1)$ coefficients, in which case all other decay channels of the volume modulus $T$ would be overwhelmed by the decays to gauge bosons, and the reheating temperature would be large. In general, the effective reheating temperature generated by decays into gauge bosons would exceed that due to decays into matter particles, (197), for any $d_{g, T} \gtrsim \mathcal{O}(1 / 40)$.

On the other hand, the decays of $T$ to gauginos are subdominant, since the corresponding decay rate is

$$
\Gamma(T \rightarrow \tilde{g} \tilde{g})=\frac{d_{g, T}^{2}}{16 \pi}\left(\frac{N_{G}}{12}\right) \frac{m_{3 / 2}^{2} M}{M_{P}^{2}} .
$$

We note that a similar suppression for the decay to gauginos was given in Ref. 75 ,

\subsection{The Number of e-Folds in Representative No-Scale Inflation Models}

In the preceding Subsection we have seen the various possibilities for reheating and the related model dependence in inflaton decay due to the reheating process. We now consider the implications for the number of e-folds $N_{*}$ in some representative no-scale models of inflation.

In the slow-roll approximation the number of e-folds to the end of inflation can be expressed as $1080[81$

$N_{*}=66.9-\ln \left(\frac{k_{*}}{R_{0} H_{0}}\right)+\frac{1}{4} \ln \left(\frac{V_{*}^{2}}{M_{P}^{4} \rho_{\mathrm{end}}}\right)+\frac{1-3 w_{\mathrm{int}}}{12\left(1+w_{\mathrm{int}}\right)} \ln \left(\frac{\rho_{\mathrm{RH}}}{\rho_{\mathrm{end}}}\right)-\frac{1}{12} \ln g_{\mathrm{RH}}$,

where $R_{0}$ and $H_{0}$ are the present cosmological scale factor and Hubble expansion rate, respectively, $V_{*}$ is the inflationary energy density at the reference scale, $\rho_{\text {end }}$ 
and $\rho_{\mathrm{RH}}$ are the energy densities at the end of inflation and after reheating, respectively, and $w_{\text {int }}$ is the e-fold average of the equation-of-state parameter during reheating. Entropy conservation after reheating has been assumed, and we refer the interested reader to Ref. 97 for details.

For Starobinsky-like models, Eq. 202 can be written in the form 97

$$
\begin{aligned}
N_{*}= & 68.66-\ln \left(\frac{k_{*}}{R_{0} H_{0}}\right)+\frac{1}{4} \ln \left(A_{s}\right)-\frac{1}{2} \ln \left(N_{*}-\sqrt{\frac{3}{8}} \frac{\phi_{\text {end }}}{M_{P}}+\frac{3}{4} e^{\sqrt{\frac{2}{3}} \frac{\phi_{\text {end }}}{M_{P}}}\right) \\
& -\frac{1}{12} \ln g_{\mathrm{RH}}+\frac{1-3 w_{\text {int }}}{12\left(1+w_{\text {int }}\right)}\left[2.030+2 \ln \left(\Gamma_{\phi} / M\right)-2 \ln \left(1+w_{\text {eff }}\right)\right. \\
& -2 \ln (0.655-1.082 \ln \delta)],
\end{aligned}
$$

where $\phi_{\text {end }}$ is the value of the inflaton field at the end of inflation, $w_{\text {eff }}$ is the time-average of the equation-of-state parameter during the reheating epoch, and $\delta$ parametrizes the degree of completion of reheating:

$$
\Omega_{\mathrm{rad}}=\frac{\rho_{\mathrm{rad}}}{\rho_{\phi}+\rho_{\mathrm{rad}}} \equiv 1-\delta .
$$

We see from 203 that $N_{*}$ depends on $\Gamma_{\phi}$ both explicitly and implicitly via the dependences in $w_{\text {int }}$ and $w_{\text {eff }}$. The e-fold-averaged equation of state parameter, $w_{\text {int }}$ in Starobinsky-like models may be fit by 9

$$
w_{\text {int }}=\frac{0.782}{\ln \left(2.096 M / \Gamma_{\phi}\right)},
$$

and the time-averaged equation of state $w_{\text {eff }} \simeq 0.27$. To calculate $N_{*}$ as a function of $\Gamma_{\phi}$, we use the Planck pivot point $k_{*}=0.05 / \mathrm{Mpc}$, corresponding to $k_{*} / R_{0} H_{0}=221$, and take the MSSM value of $g_{\mathrm{RH}}=915 / 4$. Fig. 11 displays the calculated value of $N_{*}$ over a wide range of $\Gamma_{\phi}$, parametrized by

$$
\Gamma_{\phi}=M \frac{|y|^{2}}{8 \pi}
$$

with a coupling ranging from $y=1$ to $y \sim 10^{-18}$, in which the latter would correspond to a reheating temperature $T_{\mathrm{RH}} \sim 1 \mathrm{MeV}$, below which the successful conventional BBN calculations would need to be modified substantially. Within this overall range, we indicate in Fig. 11 the values of $N_{*}$ corresponding to the decay processes (196) and (198), which are consistent with the CMB and gravitino constraints.

\section{Inflation and UV phenomenology}

So far we have seen that Starobinsky-like inflation can be constructed naturally in the context of no-scale supergravity models. The underlying $\mathrm{SU}(2,1) / \mathrm{SU}(2) \times \mathrm{U}(1)$ symmetry leads to a continuous class of phenomenological models $82[98$ in which the inflaton may be associated with either a modulus or a matter-like field. Ultimately though, we would like to be able to connect these "low" energy phenomenologies 


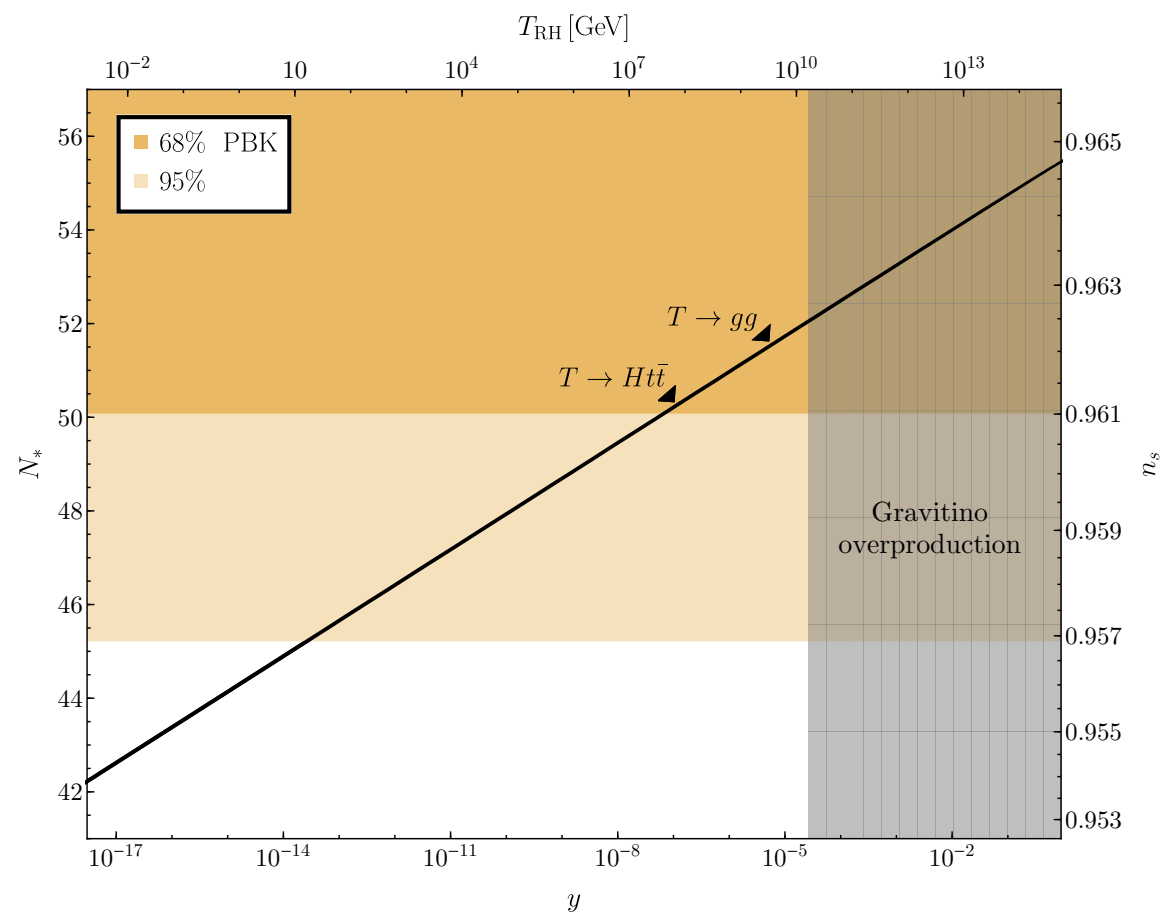

Fig. 11. The values of $N_{*}$ in no-scale Starobinsky-like models as a function of $y$ ( $\left.T_{\mathrm{RH}}\right)$, for a wide range of decay rates. The diagonal black strip corresponds to the solution of (203) in the range $10^{-3}<\delta<10^{-1}$. The right vertical axis shows the values of $n_{s}$ in Starobinsky-like noscale models, for which the tensor-to-scalar ratio varies over the range $0.0034<r<0.0057$ for $N_{*}$ in the displayed range. The horizontal light beige (orange) shaded region corresponds to the 95\% (68\%) Planck+BICEP2/Keck (PBK) CL region from Eq. (37). The vertical shaded region is excluded due to gravitino overproduction. Within it, in-medium and non-perturbative effects may also affect the inflaton decay rate. For illustrative purposes, the results corresponding to the decay processes (196) and (198) are shown.

with a UV completion of the theory. We anticipate that this should be a string theory incorporating all the gauge interactions as well as gravity. However, because the appropriate theory is not known, we are more modest in our attempts here, and examine the consequence of embedding the inflationary theory in the context of a GUT. Specifically, we consider three GUT models: SU(5), which offers a minimal way to realize the no-scale inflation in a GUT, and $\mathrm{SO}(10), 144$ neither of which can in principle be obtained from perturbative heterotic string theory, and flipped $\mathrm{SU}(5) \times \mathrm{U}(1), 130[145-148]$ which has been derived within such a string model. 


\subsection{SU(5) GUTs and No-Scale Inflation}

We first consider a supersymmetric SU(5) GUT model that incorporates no-scale inflation. As in the minimal supersymmetric SU(5), 149 three generations of SM quarks and leptons are embedded into $\mathbf{1 0}$ and $\overline{\mathbf{5}}$ representations, $\Psi_{i}$ and $\Phi_{i}$, respectively, where $i$ is the generation index, while the MSSM Higgs fields, $H_{u}$ and $H_{d}$, reside in $\mathbf{5}$ and $\overline{\mathbf{5}}$ representations, $H$ and $\bar{H}$, respectively. The SU(5) GUT symmetry is spontaneously broken by a VEV of a $\mathbf{2 4}, \Sigma$, down to the SM gauge group. In addition to these fields, we introduce an $\mathrm{SU}(5)$ singlet field, $S$, as the inflaton. We also assume that this model respects $R$-parity, so as to suppress dangerous baryon/lepton-number violating renormalizable operators; $H, \bar{H}, \Sigma$, and $S$ are $R$-parity even and the rest of the fields are $R$-parity odd.

The renormalizable superpotential for this model is given by

$$
\begin{aligned}
W_{5} & =\mu_{\Sigma} \operatorname{Tr} \Sigma^{2}+\frac{1}{6} \lambda^{\prime} \operatorname{Tr} \Sigma^{3}+\mu_{H} \bar{H} H+\lambda \bar{H} \Sigma H \\
& +\left(h_{\mathbf{1 0}}\right) \Psi \Psi H+\left(h_{\overline{\mathbf{5}}}\right) \Psi \Phi \bar{H} \\
& +\frac{M}{2} \phi^{2}-\frac{\lambda_{\phi}}{3} \phi^{3}+\lambda_{H \phi} \phi \bar{H} H+\lambda_{\phi \Sigma} \phi \operatorname{Tr} \Sigma^{2},
\end{aligned}
$$

where we have suppressed the tensor structure and omitted generation indices, for simplicity. We choose $\lambda_{\phi}=M /\left(\sqrt{3} M_{P}\right)$, so as to obtain the Starobinsky potential, ${ }^{20}$ and $\mu_{H}=3 \lambda V_{\Sigma}$, where $\langle\Sigma\rangle=V_{\Sigma} \operatorname{diag}(2,2,2,-3,-3)$ is the VEV of $\Sigma$, with $V_{\Sigma} \equiv 4 \mu_{\Sigma} / \lambda^{\prime}$, to realize doublet-triplet mass splitting for the $H$ and $\bar{H}$ multiplets. The coupling $\lambda_{\phi \Sigma}$ needs to be small in order to realize successful Starobinsky-like inflation, so we assume $\lambda_{\phi \Sigma} \ll 1$ in what follows.

The Kähler potential includes the inflaton field as an untwisted field. The rest of the fields can be included in the Kähler potential as either untwisted or twisted fields; for concreteness, we assume all of the fields are untwisted in the following discussion.

All of the above fields except $\phi$ and $\Sigma$ have vanishing field values in the instantaneous potential minimum during inflation. We assume that the adjoint Higgs field is displaced by a small amount from its vacuum value during the inflationary period, i.e.,

$$
\langle\Sigma\rangle=\left(V_{\Sigma}+\sigma\right) \operatorname{diag}(2,2,2,-3,-3),
$$

with $|\sigma| \ll V_{\Sigma}$. We show below that this condition can be satisfied for sufficiently small $\lambda_{\phi \Sigma}$. In this case, the scalar potential during inflation is given by

$$
V=\frac{\hat{V}}{\left(T+T^{*}-|\phi|^{2} / 3-10\left|V_{\Sigma}+\sigma\right|^{2}\right)^{2}},
$$

with

$$
\begin{aligned}
\hat{V} & =\left|M \phi-\lambda_{\phi} \phi^{2}+30 \lambda_{\phi \Sigma}\left(V_{\Sigma}+\sigma\right)^{2}\right|^{2} \\
& +\frac{15}{2}\left|V_{\Sigma}+\sigma\right|^{2}\left|4\left(\mu_{\Sigma}+\lambda_{\phi \Sigma} \phi\right)-\lambda^{\prime}\left(V_{\Sigma}+\sigma\right)\right|^{2} .
\end{aligned}
$$


The instantaneous value of $\Sigma$ during inflation is determined by the second term in the above equation for $\lambda_{\phi \Sigma} \ll 1$ :

$$
\sigma \simeq \frac{4 \lambda_{\phi \Sigma} \phi}{\lambda^{\prime}}
$$

To assure $|\sigma| \ll V_{\Sigma}$, we thus assume $\lambda_{\phi \Sigma} \ll \mu_{\Sigma} / M_{P}$. This condition is generically weaker than the limit obtained from the bounds on the inflation parameters, as we see below.

With $\langle T\rangle=1 / 2$ and the canonically-normalized field $x$ given by Eq. 120 , the inflaton potential can be approximated by

$$
V \simeq \frac{3}{4} M^{2}\left(1-e^{-\sqrt{\frac{2}{3}} x}\right)^{2}+\Delta V
$$

where

$$
\begin{aligned}
\Delta V & =15 M^{2} V_{\Sigma}^{2} e^{-\sqrt{\frac{2}{3}} x} \sinh ^{2}\left(\sqrt{\frac{2}{3}} x\right) \\
& +60 \sqrt{3} \lambda_{\phi \Sigma} M V_{\Sigma}^{2} e^{-\frac{1}{\sqrt{6}} x} \cosh ^{2}\left(\frac{x}{\sqrt{6}}\right) \sinh \left(\frac{x}{\sqrt{6}}\right) \\
& \simeq \frac{3}{4} M^{2} V_{\Sigma}^{2}\left(5+\frac{30 \lambda_{\phi \Sigma}}{\sqrt{3} M}\right) e^{\sqrt{\frac{2}{3}} x} .
\end{aligned}
$$

The CMB observables are then estimated to take the values

$$
\begin{aligned}
n_{s} & \simeq 1-\frac{2}{N_{*}}+\frac{320}{27}\left(\frac{V_{\Sigma}}{M_{P}}\right)^{2}\left(1+\frac{2 \sqrt{3} \lambda_{\phi \Sigma} M_{P}}{M}\right) N_{*}, \\
r & \simeq \frac{12}{N_{*}^{2}}+\frac{640}{9}\left(\frac{V_{\Sigma}}{M_{P}}\right)^{2}\left(1+\frac{2 \sqrt{3} \lambda_{\phi \Sigma} M_{P}}{M}\right),
\end{aligned}
$$

where we explicitly exhibit factors of the Planck mass, which were often set to unity in previous expressions. We see that the predicted values of $n_{s}$ and $r$ deviate from the Starobinsky values. These deviations are constrained by the Planck measurement on $n_{s} \stackrel{10}{10}$ For $N_{*}=50(60)$, for instance, we have

$$
\left(1+\frac{2 \sqrt{3} \lambda_{\phi \Sigma} M_{P}}{M}\right)^{1 / 2} V_{\Sigma}<1.1(0.72) \times 10^{16} \mathrm{GeV},
$$

at $95 \%$ CL. This estimate shows that the CMB measurement can probe GUT physics directly in this scenario and that, in fact, the Planck measurement has already imposed a severe limit on the GUT scale. Note that this bound exists even if the inflaton has no direct coupling to the GUT Higgs in the superpotential; as we can see in Eq. 213, $\Delta V \neq 0$ for $\lambda_{\phi \Sigma}=0$. This contribution comes from the overall factor of $e^{2 K / 3}$ in the scalar potential when there are fields that have non-vanishing VEVs during inflation. This type of contribution exists also in the $\mathrm{SO}(10)$ model discussed in Section 12.2, but is absent in the flipped SU(5) model discussed in Section 12.3 .

The bound (216) has important implications for the allowed region in the MSSM parameter space in the minimal scenario, since the value of $V_{\Sigma}$ is determined by 
a renormalization-group analysis. Using threshold corrections at the GUT scale, we can determine a combination of the masses of the GUT-scale particles, 150,152 namely $\left(M_{X}^{2} M_{\Sigma}\right)^{1 / 3}$, where $M_{X}$ and $M_{\Sigma}$ are the masses of the GUT gauge field and the adjoint Higgs field, respectively. The VEV of the GUT Higgs is then given by

$$
V_{\Sigma}=\frac{1}{5}\left(\frac{2}{\lambda^{\prime} g_{5}^{2}}\right)^{1 / 3}\left(M_{X}^{2} M_{\Sigma}\right)^{1 / 3}
$$

where $g_{5}$ is the $\mathrm{SU}(5)$ gauge coupling, which is also determined by GUT threshold conditions. As a result, $V_{\Sigma}$ is given as a function of a free parameter $\lambda^{\prime}$ once the MSSM mass spectrum is fixed. The bound (216) then leads to a lower limit on $\lambda^{\prime}$, which provides an additional restriction on the parameter space, especially in the constrained scenarios discussed in Refs. 37, 124, 153.

In the present scenario, reheating proceeds through the coupling $\lambda_{H S}$ in Eq. 207), with which the inflaton decays into $H_{u}$ and $H_{d}$. The decay rate is

$$
\Gamma\left(\phi \rightarrow H_{u} H_{d}\right) \simeq 2 \times \frac{\left|\lambda_{H \phi}\right|^{2}}{8 \pi} M .
$$

As discussed in Section 11.5.1, the coupling $\left|\lambda_{H S}\right|$ must be $\left|\lambda_{H S}\right| \lesssim 10^{-5}$ in order to evade the gravitino overproduction problem. If we also introduce right-handed neutrinos to this model, which are $\mathrm{SU}(5)$ singlets with $R$-parity odd, we can couple the inflaton also to these fields without modifying the inflation dynamics. In this case, the inflaton can decay into right-handed neutrinos as well, and the gravitino overproduction bound again restricts the inflaton-right-handed neutrino couplings to be $\lesssim 10^{-5}$.

\subsection{SO(10) GUTs and No-Scale Inflation}

We saw in Section 11.5.1 that efficient reheating is possible when we identify the inflaton with the right-handed sneutrino 123 136 One might think that this identification could carry over to an $\mathrm{SO}(10)$ completion, in which the right-handed neutrino is included with other Standard Model fields in the $\mathbf{1 6}$ representation. However, this is not possible as there are no gauge-invariant $\mathbf{1 6}^{2}$ or $\mathbf{1 6}^{3}$ couplings in $\mathrm{SO}(10)$. The $\mathbf{5 4}$ or $\mathbf{2 1 0}$ representations do allow both quadratic and cubic couplings in the superpotential, though these are typically associated with Higgs fields that break GUT symmetry, and would require $M$ to be GUT scale $\sim 10^{16} \mathrm{GeV}$, rather than $\sim 10^{13} \mathrm{GeV}$ as expected for the inflaton. Also, they acquire GUT-scale VEVs, which is also not the case for the Starobinsky model.

The model we consider $\frac{144}{14}$ includes an $\mathrm{SO}(10)$ singlet as the inflaton. The field content is similar to the $\mathrm{SO}(10)$ GUT in Ref. 154, which includes a 210 representation, $\Sigma$, to break $\mathrm{SO}(10)$ to an intermediate gauge group, a pair of $\mathbf{1 6}$ and $\overline{\mathbf{1 6}}$ representations, $\Phi$ and $\bar{\Phi}$, to break the intermediate gauge group to the SM, a 10 representation, $H$, which includes the SM Higgs fields that break the Standard 
Model. There are also three matter multiplets, $\psi_{i}$, in $\mathbf{1 6}$ representations, and we include one singlet per generation, $\phi_{i}$, one of which is identified as the inflaton.

The superpotential of the theory takes the following generic form:

$$
\begin{aligned}
W & =M\left(\frac{1}{2} \phi^{2}-\frac{1}{3 \sqrt{3}} \phi^{3}\right)+y H \psi \psi+\left(M^{\prime}+b \phi\right) \bar{\Phi} \psi \\
& +m_{\Phi} \bar{\Phi} \Phi+\frac{\eta}{4 !} \bar{\Phi} \Phi \Sigma+\frac{m_{\Sigma}}{4 !} \Sigma^{2}+\frac{\Lambda}{4 !} \Sigma^{3}+m_{H} H^{2}+\lambda_{\phi H} \phi H^{2} \\
& +H\left(\alpha \Phi \Phi+\bar{\alpha} \bar{\Phi} \bar{\Phi}+\alpha^{\prime} \Phi \psi\right)+c \phi \bar{\Phi} \Phi+\frac{b^{\prime}}{4 !} \bar{\Phi} \psi \Sigma+\frac{\gamma}{4 !} \phi \Sigma^{2}+\kappa,
\end{aligned}
$$

where we have again suppressed the tensor structure and omitted generation indices, in the interest of simplicity, and do not discuss here the possibility of mixing between the singlet superfields $\phi_{i}$.

The first two terms in 219) are a Wess-Zumino superpotential that reproduces Starobinsky inflation in no-scale supergravity, 20,82 and the third term represents the SM Yukawa couplings. The fourth term includes a coupling between SM fields and the inflaton $\phi$, as does the tenth term: these couplings fix the neutrino masses and determine the inflaton decay rate. The couplings in the fifth through eighth terms enable the SM singlet components of $\Phi, \bar{\Phi}$, and $\Sigma$ to acquire non-vanishing VEVs. When these VEVs develop, the terms $\alpha H \Phi \Phi$ and $\bar{\alpha} \bar{H} \bar{\Phi} \bar{\Phi}$ mix the $\mathrm{SU}(2)_{L}$ doublet components of the $H, \Phi$, and $\bar{\Phi}$ multiplets. Suitable choices of these couplings realize doublet-triplet splitting by enabling two linear combinations of these fields, denoted by $H_{u}$ and $H_{d}$, to have masses far below the GUT and intermediate scales $\frac{155}{15}$ The VEVs of $H_{u}$ and $H_{d}$ break electroweak symmetry at the TeV scale, as in the MSSM. A weak-scale gravitino mass is obtained for a suitable value of the constant $\kappa$, through the relation $m_{3 / 2}=\left\langle e^{K / 2} W\right\rangle$. This supersymmetry breaking may be generated by a separate Polonyi sector ${ }^{22}$ as discussed previously. Finally, the noscale Kähler potential of the $\mathrm{SO}(10)$ model is taken to be

$$
K=-3 \ln \left[T+T^{*}-\frac{1}{3}\left(\phi^{*} \phi+H^{\dagger} H+\psi^{\dagger} \psi+\Phi^{\dagger} \Phi+\bar{\Phi}^{\dagger} \bar{\Phi}+\frac{1}{4 !} \Sigma^{\dagger} \Sigma\right)\right]
$$

A full treatment of this model is given in Ref. 144, and here we simply review the highlights.

We parametrize the VEVs of the SM singlets in the Higgs representation by

$$
\begin{aligned}
& p=\langle\Sigma(\mathbf{1}, \mathbf{1}, \mathbf{1})\rangle, \quad a=\langle\Sigma(\mathbf{1 5}, \mathbf{1}, \mathbf{1})\rangle, \quad \omega=\langle\Sigma(\mathbf{1 5}, \mathbf{1}, \mathbf{3})\rangle, \\
& f_{R}=\langle\Phi(\overline{\mathbf{4}}, \mathbf{1}, \mathbf{2})\rangle, \quad \bar{f}_{R}=\langle\bar{\Phi}(\mathbf{4}, \mathbf{1}, \mathbf{2})\rangle, \quad \widetilde{\nu}_{R}=\langle\psi(\overline{\mathbf{4}}, \mathbf{1}, \mathbf{2})\rangle,
\end{aligned}
$$

where we show explicitly their $\mathrm{SU}(4)_{C} \otimes \mathrm{SU}(2)_{L} \otimes \mathrm{SU}(2)_{R}$ quantum numbers. We assume $\widetilde{\nu}_{R}=0$ at the minimum. This is stable with a positive mass-squared if either $b$ or $b^{\prime}$ is non-zero. To study better the scalar potential, we write the superpotential 
219 in terms of the SM singlet fields, with the rest of the fields set to zero:

$$
\begin{aligned}
W & =M\left(\frac{1}{2} \phi^{2}-\frac{1}{3 \sqrt{3}} \phi^{3}\right)-\left(M^{\prime}+b \phi\right) \bar{f}_{R} \nu_{R}+\left(\eta f_{R}+b^{\prime} \nu_{R}\right) \bar{f}_{R}(p+3 a+6 \omega) \\
& -\left(m_{\Phi}+c \phi\right) \bar{f}_{R} f_{R}+\left(m_{\Sigma}+\gamma \phi\right)\left(p^{2}+3 a^{2}+6 \omega^{2}\right)+2 \Lambda\left(a^{3}+3 p \omega^{2}+6 a \omega^{2}\right)+\kappa .
\end{aligned}
$$

In order to realize inflation, the couplings $b, c$, and $\gamma$ must be small, as all of them break the scale symmetry associated with the potential. Here, we take $c=\gamma=0$. We shall see that $b$ enters into the neutrino mass matrix, and we show the effects of $b \neq 0$ on the inflaton potential. The Higgs fields are displaced a negligible amount from their vacuum values during inflation, and the scalar potential during inflation takes the simple form

$$
V \simeq \frac{\hat{V}}{\left[1-\frac{1}{3}\left(|\phi|^{2}+\Delta K\right)\right]^{2}}
$$

where $f \equiv\left|f_{R}\right|=\left|\bar{f}_{R}\right|$ and

$$
\begin{aligned}
\hat{V} & =M^{2}\left|\phi-\phi^{2} / \sqrt{3}\right|^{2}+|\phi|^{2}|b f|^{2}, \\
\Delta K & \equiv|p|^{2}+3|a|^{2}+6|\omega|^{2}+2|f|^{2} .
\end{aligned}
$$

In terms of the canonically-normalized field $x$, the scalar potential takes the form

$$
V \simeq \frac{3}{4} M^{2}\left(1-e^{-\sqrt{2 / 3} x}\right)^{2}+\Delta V
$$

where

$$
\Delta V=\left[\frac{3}{4}|b f|^{2}+\frac{1}{2} M^{2} e^{-\sqrt{2 / 3} x} \Delta K\right] \sinh ^{2}(\sqrt{2 / 3} x) .
$$

We show in Fig. 12 the effects of the coupling $b$ in $\Delta V$, plotting the tilt of the scalar perturbation spectrum, $n_{s}$, and the tensor-to-scalar ratio, $r$. The red (pink) shaded regions correspond to the 68 (95) \% CL limits from Planck. ${ }^{10}$ In the limit where $|b f|, \Delta K \ll 1$, the inflationary parameters can be approximated analytically by $\frac{144}{14}$

$$
\begin{aligned}
n_{s} & \simeq 1-\frac{2}{N_{*}}+\frac{8}{3}\left(\frac{b f}{M}\right)^{2} N_{*}^{2}+\frac{32}{81} N_{*} \Delta K, \\
r & \simeq \frac{12}{N_{*}^{2}}+\frac{32}{3}\left(\frac{b f}{M}\right)^{2} N_{*}+\frac{64}{27} \Delta K .
\end{aligned}
$$

Figs. 2 and 4 in Ref. 144 show the effects of the term proportional to $\Delta K$, and set an upper limit on this combination of $10^{-3.1}$. Notice that, similarly to (216), this limit is present even if the inflaton does not couple to the GUT Higgs fields in the superpotential. If the values of $p, a, \omega, f$ are $\simeq V_{\mathrm{GUT}}, \Delta K \simeq 12 V_{\mathrm{GUT}}^{2}$, and this limit gives $V_{\mathrm{GUT}} \lesssim 2 \times 10^{16} \mathrm{GeV}$.

We see in Fig. 12 the effect of a non-zero value of $b$, recalling that $b f=0$ corresponds to the exact Starobinsky result. In order to obtain values of $\left(n_{s}, r\right)$ consistent with Planck, we must require that the product bf $<10^{-7.9}\left(10^{-8.1}\right)$ 


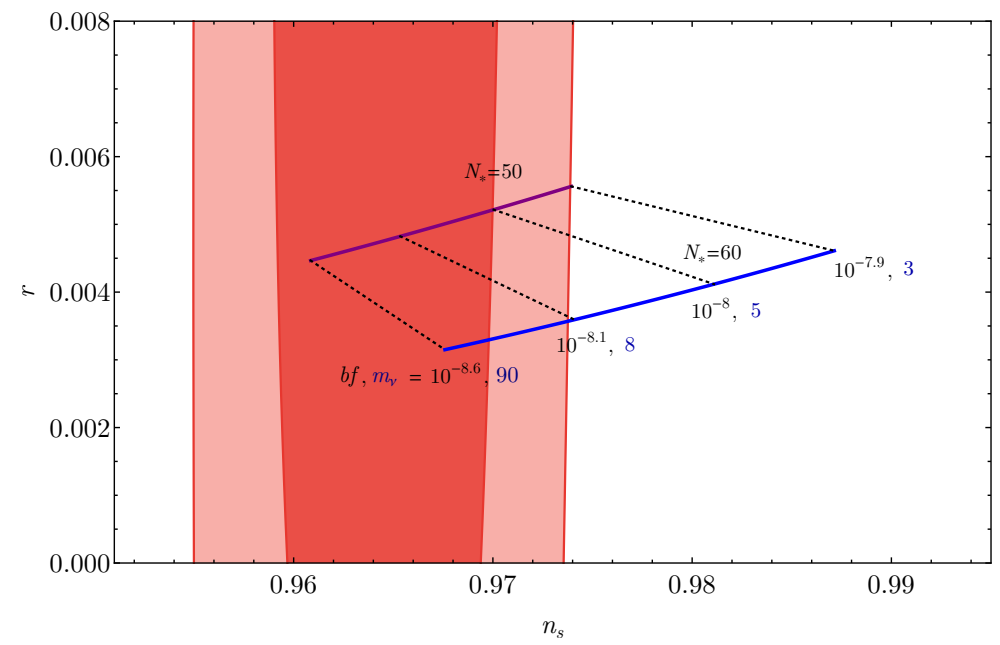

Fig. 12. Parametric $\left(n_{s}, r\right)$ curves as functions of bf for $N_{*}=50,60$, with the 68 and $95 \% C L$ Planck constraints shown in the background. The solid curves illustrate the parametric dependence using the analytical approximation (226) and (227) with $\Delta K=0$. The dotted curves illustrate particular values of $b f$, quantified in units of $M_{P}$, and we indicate the corresponding left-handed neutrino masses in units of $10^{-4} \mathrm{eV}$, assuming $y_{\nu} \sin \beta=10^{-5}$ and $M=10^{-5} M_{P}$.

for $N_{*} \simeq 50(60) e$-folds of inflation. Since the VEV of $\Phi$ is less than the GUT scale, $f \lesssim 10^{-2.1}$, and the constraint we have on $b$ is $b<10^{-5.8}\left(10^{-6}\right)$. The scalar potential for several choices of $b f$ is shown in Fig. 13 . As one can see, so long as bf $\lesssim 10^{-2.5} M \sim 10^{-7.5}$, the potential is indistinguishable from the Starobinsky potential out to the value $x \sim 5.5$ needed for $60 e$-folds of inflation.

Reheating in this model is largely controlled by the coupling $b$. The inflaton decay rate is

$$
\Gamma\left(\phi \rightarrow H_{u} \tilde{L}\right)+\Gamma\left(\phi \rightarrow \tilde{H}_{u} L\right) \simeq \frac{M}{4 \pi}|b|^{2},
$$

which leads to a reheating temperature

$$
T_{\mathrm{RH}} \simeq 10^{15} \mathrm{GeV} \times|b| \lesssim 10^{9} \mathrm{GeV},
$$

compatible with the success of conventional BBN.

We note also that $\mathrm{CP}$ violation in the inflaton decay process may generate a lepton asymmetry non-thermally $46 \mid 137$ which is then converted to a baryon asym-

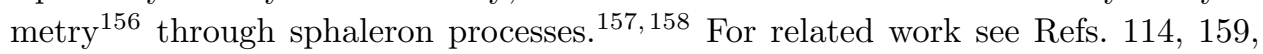
160, 161, 162, 163, 164 .

Before we conclude this Subsection, we comment on the generation of neutrino masses in this model. A non-zero value of the coupling $b$ induces mixing between right-handed neutrinos and the "singlinos" $\tilde{\phi}_{i}$, which are the fermionic components of the singlet superfields $\phi_{i}$. Disregarding Planck-suppressed factors, the neutrino- 


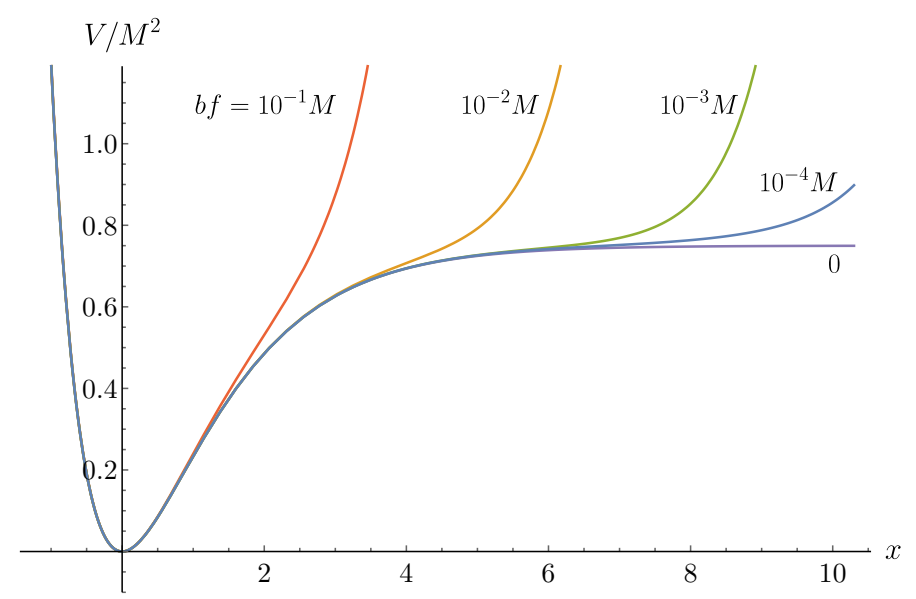

Fig. 13. The inflationary potential for different values of $b f$, in units of the inflaton mass $M \simeq$ $10^{-5} M_{P}$. The curve labeled $b f=0$ is the Starobinsky potential.

singlino fermion mass matrix is given by 154

$$
\mathcal{L}_{\text {mass }}=-\left(\bar{\nu}_{L} \bar{\nu}_{R}^{c} \overline{\tilde{\phi}}\right)\left(\begin{array}{ccc}
0 & -y_{\nu} v \sin \beta & 0 \\
-y_{\nu} v \sin \beta & 0 & -b f \\
0 & -b f & M
\end{array}\right)\left(\begin{array}{c}
\nu_{L} \\
\nu_{R}^{c} \\
\tilde{\phi}
\end{array}\right)
$$

A similar form for the mass matrix is found in flipped SU $(5) \sqrt{165} \mid 166$ (see the following Section). For the first-generation neutrinos, sufficient inflation restricts the coupling $b$ as we have seen previously. In this case, the couplings satisfy the hierarchy

$$
y_{\nu} v \sin \beta \ll b f \ll M,
$$

and the fermion mass eigenstates are

$$
\begin{aligned}
\nu_{L}^{M} & \simeq \nu_{L}-\frac{M y_{\nu} v \sin \beta}{(b f)^{2}} \nu_{R}^{c}-\frac{y_{\nu} v \sin \beta}{b f} \tilde{\phi} \\
\nu_{R}^{M} & \simeq \nu_{R}^{c}+\frac{b f}{M} \tilde{\phi}+\frac{M y_{\nu} v \sin \beta}{(b f)^{2}} \nu_{L} \\
\tilde{\phi}^{M} & \simeq \tilde{\phi}-\frac{b f}{M} \nu_{R}^{c}+\frac{b f y_{\nu} v \sin \beta}{M^{2}} \nu_{L} .
\end{aligned}
$$

For the second and third generations, the coupling $b$ (recall we have suppressed all generation indices) can be arbitrary. For all generations, the masses of the light neutrinos are given by

$$
m_{\nu} \simeq M\left(\frac{y_{\nu} v \sin \beta}{b f}\right)^{2} \simeq\left(\frac{M}{10^{-5} M_{P}}\right)\left(\frac{y_{\nu} \sin \beta}{10^{-5}}\right)^{2}\left(\frac{b f}{10^{-8} M_{P}}\right)^{-2} \times 10^{-4} \mathrm{eV} .
$$

As we see from this equation, we cannot take $b f$ to be arbitrary small; to obtain a sufficiently small neutrino mass, $m_{\nu} \lesssim 0.1 \mathrm{eV}$, we need $|b f| \gtrsim 3.5 \times 10^{-10} M_{P}$ for 
$M=10^{-5} M_{P}$ and $y_{\nu} \sin \beta=10^{-5}$, which is approximately equal to the size of the up-quark Yukawa coupling. If we instead take $f_{\nu} \sin \beta \simeq 10^{-2}$, corresponding to the charm-quark Yukawa coupling, the condition $m_{\nu} \lesssim 0.1 \mathrm{eV}$ leads to $|b f| \gtrsim 3.5 \times 10^{-7}$, which conflicts with the bound set by the Planck observation, as shown in Fig. 12 We thus conclude that in this model the inflaton field $\phi$ must predominantly couple to the first-generation neutrino.

This coupling yields a strong correlation between the first-generation neutrino mass and the inflation parameters $n_{s}$ and $r$ as shown in Fig. 12. It becomes apparent if we write Eqs. (228) and (229) using Eq. 237) as

$$
\begin{aligned}
n_{s} & \simeq 1-\frac{2}{N_{*}}+\frac{8}{3} \frac{\left(y_{\nu} v \sin \beta\right)^{2}}{M m_{\nu}} N_{*}^{2}+\frac{32}{81} N_{*} \Delta K, \\
r & \simeq \frac{12}{N_{*}^{2}}+\frac{32}{3} \frac{\left(y_{\nu} v \sin \beta\right)^{2}}{M m_{\nu}} N_{*}+\frac{64}{27} \Delta K .
\end{aligned}
$$

As we see, both $n_{s}$ and $r$ get larger for a smaller neutrino mass.

\subsection{Flipped $S U(5) \times U(1)$ and No-Scale Inflation}

Another possible UV completion of the no-scale inflationary scenario is a flipped $\mathrm{SU}(5) \times \mathrm{U}(1) \mathrm{GUT} 21] 165|167| 168$ Unlike the previous $\mathrm{SU}(5)$ and $\mathrm{SO}(10)$ GUT models, it can be derived in the fermionic formulation of weakly-coupled heterotic string theory.21 This is because, unlike the two previous models, it does not require an adjoint Higgs or larger Higgs representation for gauge symmetry breaking.

The flipped $\mathrm{SU}(5) \times \mathrm{U}(1)$ GUT contains three generations of SM matter fields, each with a right-handed neutrino, which are each placed in $\mathbf{1 0}, \overline{\mathbf{5}}$, and $\mathbf{1}$ representations of $\mathrm{SU}(5)$. The representation assignments of the right-handed leptons and the right-handed up- and down-type quarks, are "flipped" with respect to standard $\mathrm{SU}(5)$. Moreover, the $I= \pm 1 / 2$ partners in the left-handed lepton and quark doublets are also flipped. The $\mathrm{SU}(5) \times \mathrm{U}(1)$ GUT group is broken to the $\mathrm{SM}$ via $\mathbf{1 0}+\overline{\mathbf{1 0}}$ Higgs representations of $\mathrm{SU}(5)$, and subsequently to the unbroken $\mathrm{SU}(3) \times \mathrm{U}(1)$ symmetry via electroweak doublets in $\mathbf{5}+\overline{\mathbf{5}}$ representations. Our notations for the fields and their gauge representations are as follows:

$$
\begin{aligned}
& F_{i}=(\mathbf{1 0}, 1)_{i} \ni\left\{d^{c}, Q, \nu^{c}\right\}_{i}, \\
& \bar{f}_{i}=(\overline{\mathbf{5}},-3)_{i} \ni\left\{u^{c}, L\right\}_{i}, \\
& \ell_{i}^{c}=(\mathbf{1}, 5)_{i} \quad \ni\left\{e^{c}\right\}_{i}, \\
& H=(\mathbf{1 0}, 1), \quad \bar{H}=(\overline{\mathbf{1 0}},-1) \text {, } \\
& h=(\mathbf{5},-2), \bar{h}=(\overline{\mathbf{5}}, 2) \text {, }
\end{aligned}
$$

where the subscripts $i=1,2,3$ are generation indices that we suppress unless they are necessary. The model contains four singlet fields, which have no U(1) charges and are denoted by $\phi_{a}=(\mathbf{1}, 0), a=0, \ldots, 3$. 
The superpotential up to third order in the chiral superfields is:

$$
\begin{aligned}
W= & \lambda_{1}^{i j} F_{i} F_{j} h+\lambda_{2}^{i j} F_{i} \bar{f}_{j} \bar{h}+\lambda_{3}^{i j} \bar{f}_{i} \ell_{j}^{c} h+\lambda_{4} H H h+\lambda_{5} \bar{H} \bar{H} \bar{h} \\
& +\lambda_{6}^{i a} F_{i} \bar{H} \phi_{a}+\lambda_{7}^{a} h \bar{h} \phi_{a}+\lambda_{8}^{a b c} \phi_{a} \phi_{b} \phi_{c}+\mu^{a b} \phi_{a} \phi_{b},
\end{aligned}
$$

where the indices $i, j$ run over the three fermion families, and we have suppressed gauge group indices. We impose a $\mathbb{Z}_{2}$ symmetry $H \rightarrow-H$ that prevents the mixing of SM matter fields with Higgs colour triplets and elements of the Higgs decuplets. This symmetry also suppresses the supersymmetric mass term for $H$ and $\bar{H}$, and thus suppresses dimension-five proton decay operators.

The first 3 terms of the superpotential (241) provide the Standard Model Yukawa couplings. The fourth and fifth terms provide for the doublet-triplet separation. The sixth term accounts for neutrino masses. The seventh term plays the role of the MSSM $\mu$-term. The last two terms determine the inflationary potential and also play roles in neutrino masses. Without loss of generality, we take $\lambda_{2}^{i j}$ and $\mu^{a b}$ to be real and diagonal in what follows.

There is one linear combination of the Standard Model singlet components $\nu_{H}^{c}$ and $\nu_{\bar{H}}^{c}$ in $H$ and $\bar{H}$, respectively, corresponding to a $D$ - and $F$-flat direction in the potential, that is massless in the supersymmetric limit. This combination is denoted by $\Phi$, and referred to as the flaton, and $\mathrm{SU}(5) \times \mathrm{U}(1)$ GUT symmetry is broken along this direction. A soft supersymmetry-breaking mass term destabilizes the symmetric value $\Phi=0$, and the degeneracy along this flat direction is also lifted by a non-renormalizable superpotential term of the form

$$
W_{\mathrm{NR}}=\frac{\lambda}{n ! M_{P}^{2 n-3}}(H \bar{H})^{n},
$$

so that the effective potential for the flaton field takes the form

$$
V_{\text {non-th }}(\Phi)=V_{0}-\frac{1}{2} m_{\Phi}^{2} \Phi^{2}+\frac{|\lambda|^{2}}{[(n-1) !]^{2} M_{P}^{4 n-6}} \Phi^{4 n-2},
$$

where $m_{\Phi}$ denotes the soft mass of $\Phi$. Minimizing the potential, we find

$$
\langle\Phi\rangle=\left[\frac{\{(n-1) !\}^{2} m_{\Phi}^{2} M_{P}^{4 n-6}}{(4 n-2)|\lambda|^{2}}\right]^{\frac{1}{4(n-1)}} .
$$

Therefore, to obtain a GUT-scale VEV with a $\lambda=\mathcal{O}(1)$, we should have $n \geq 4$, and here we take $n=4$. The absence of unwanted terms, e.g., $(H \bar{H})^{2}$, may be attributed to an additional symmetry, such as $R$ symmetry 169 With the flat direction lifted, the flaton (and flatino) mass is of the order of the supersymmetry-breaking scale. For further details, see Ref. 145.

We concentrate here on the strong reheating scenario discussed in Ref. 130, in which GUT symmetry is unbroken at the end of inflation, and also assume that the GUT symmetry remains unbroken during reheating. In this scenario the GUT phase transition arises because the number of light degrees of freedom, $g$, differs between the broken and unbroken phases, $130[145,[170,172]$ and massless superfields make 
a temperature-dependent correction $-g \pi^{2} T^{4} / 90$ to the effective potential. Because $g=103$ in the unbroken phase, compared to $g=62$ in the broken phase, at high temperatures $\Phi$ is stabilized at the origin. However, when the temperature drops below the $\mathrm{SU}(5)$ confinement scale, $\Lambda_{c}$, the number of light degrees of freedom decreases significantly to $g \leq 25$, favoring the broken phase. ${ }^{145}$ In this strong reheating scenario the phase transition is driven by the incoherent component of the flaton if $\Lambda_{c} \gtrsim 2.3\left(m_{\Phi} M_{\mathrm{GUT}}\right)^{1 / 2} 1130$ This condition becomes $\Lambda_{c} \gtrsim 2.3 \times 10^{10} \mathrm{GeV}$ for $m_{\Phi}=10^{4} \mathrm{GeV}$ and $M_{\mathrm{GUT}}=10^{16} \mathrm{GeV}$.

Figure 14 shows the shape of the effective potential as a function of $\Phi$ for $0.03 \leq$ $T / \Lambda_{c} \leq 1.2 .130$ We note that the minimum near the origin is metastable for $1 \gtrsim$ $T / \Lambda_{c} \gtrsim 0.03$, separated from the true vacuum by a shrinking barrier that finally disappears for $T \lesssim 0.03 \Lambda_{c}$.

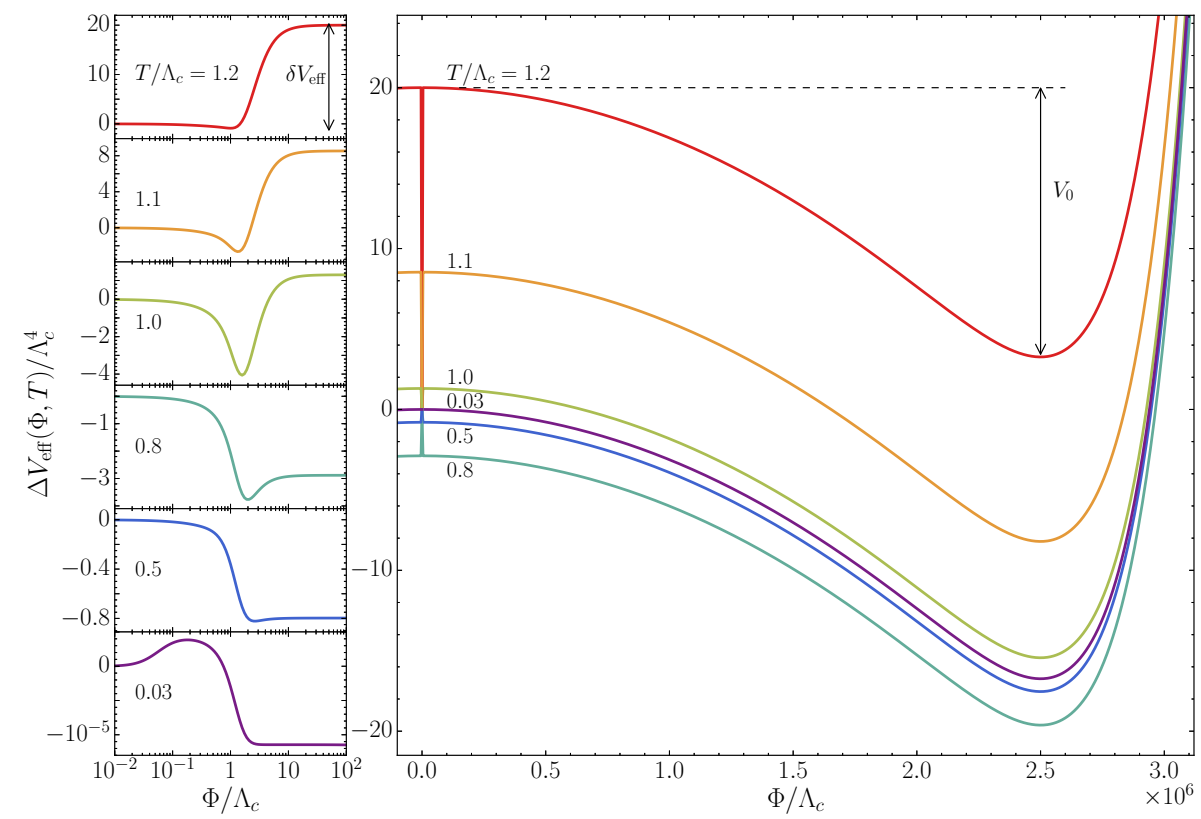

Fig. 14. The evolution with temperature of the effective potential in strongly-coupled $S U(5) \times U(1) ! 130$ Here $\Delta V_{\text {eff }}=V_{\text {eff }}(\Phi, T)-V_{\text {eff }}(0, T)$, where $V_{\text {eff }}$ includes the non-thermal contribution 243) with $n=4, m_{\Phi}=10 \mathrm{TeV}, \Lambda_{c}=4 \times 10^{9} \mathrm{GeV}$ (for which $\delta V_{\text {eff }} \sim V_{0}$ ) and $\langle\Phi\rangle=2.5 \times 10^{6} \Lambda_{c}$ at low temperature. The heights of the left and right sides of the barrier $\delta V_{\mathrm{eff}}$ and $V_{0}$ are labelled for $T / \Lambda_{c}=1.2$.

In the case of strong reheating, the flaton decouples from the thermal bath. When $T \lesssim m_{\Phi}$ it becomes non-relativistic and eventually dominates the energy density of the Universe until it decays. Flatons decay when the Hubble expansion rate is comparable to the flaton decay rate, $\Gamma_{\Phi}$, releasing, in general, a large amount 
of entropy, which is estimated to be 130 146

$$
\Delta \simeq 1.6 \times 10^{4} \lambda_{1,2,3,7}^{-2}\left(\frac{M_{\mathrm{GUT}}}{10^{16} \mathrm{GeV}}\right)\left(\frac{10 \mathrm{TeV}}{m_{\mathrm{soft}}^{2} / m_{\Phi}}\right)^{1 / 2}
$$

where $m_{\text {soft }}$ represents a typical sfermion mass. The Universe is reheated again, this time to a temperature $T_{\mathrm{RH}}^{\prime} \propto\left(\Gamma_{\Phi} M_{P}\right)^{1 / 2}$.

As in the case of $\mathrm{SO}(10)$, the inflaton is identified with one (the lightest) of the singlets, $\phi_{a}$, which we denote simply by $\phi$. As noted above, we take $\mu^{a b}$ to be diagonal,

$$
\mu^{a b}=\operatorname{diag}\left(M / 2, \mu^{11}, \mu^{22}, \mu^{33}\right), \quad \mu^{a b} \leq M_{\mathrm{GUT}} .
$$

For Starobinsky inflation, the coupling $\lambda_{8}$ must satisfy

$$
-3 \sqrt{3} \lambda_{8}^{000}=M
$$

in which case the potential for the SM singlet fields is

$$
\begin{aligned}
V_{F} \simeq & \frac{3}{4} M^{2}\left(1-e^{-\sqrt{2 / 3} x}\right)^{2}+\frac{3}{4} \sinh ^{2}(\sqrt{2 / 3} x) \sum_{i}\left|\lambda_{6}^{i 0}\right|^{2}\left(\left|\tilde{\nu}_{\bar{H}}^{c}\right|^{2}+\left|\tilde{\nu}_{i}^{c}\right|^{2}\right) \\
& +\frac{1}{8} M^{2} e^{\sqrt{2 / 3} x}\left(\left|\tilde{\nu}_{\bar{H}}^{c}\right|^{2}+\sum_{i}\left|\tilde{\nu}_{i}^{c}\right|^{2}\right)+\cdots,
\end{aligned}
$$

where $x$ is the canonically-normalized inflaton once again. As one can see, the large VEV for $x$ during inflation produces a mass term for $\tilde{\nu}^{c}$ and the GUT-breaking field $\tilde{\nu}_{H}^{c}$ that are then driven to the origin as assumed above.

It was shown in Ref. 130 that reheating is completed in the symmetric phase if $\left|\lambda_{6}^{i 0}\right| \gtrsim \mathcal{O}\left(10^{-4}\right)$. When $\langle\Phi\rangle<M$, the inflaton decays to $F$ and $\bar{H}$, with a rate given by

$$
\Gamma\left(x \rightarrow F_{i} \bar{H}\right) \simeq 10 \times \frac{\left|\lambda_{6}^{i 0}\right|^{2}}{8 \pi}\left(1-\frac{\langle\Phi\rangle^{2}}{M^{2}}\right) M
$$

The reheating temperature in this case is given by

$$
T_{\mathrm{RH}} \simeq 1.7 \times 10^{15} \mathrm{GeV} \times \sqrt{\sum_{i}\left|\lambda_{6}^{i 0}\right|^{2}}
$$

indicating a direct relation between $T_{\mathrm{RH}}$ and $\lambda_{6}$. This coupling is similar in nature to $b$ in the $\mathrm{SO}(10)$ GUT model discussed in the previous Section.

During reheating, gravitinos are produced via the scattering and decay of particles in the thermal bath $44,56,58-65$ For the calculation of the gravitino production rate, we use the formalism outlined in Ref. 63, but with the group-theoretical factors and couplings appropriate to flipped $\mathrm{SU}(5) \times \mathrm{U}(1)$. As discussed in Section 11.5 . these gravitinos eventually decay into LSPs, making a non-thermal contribution to 
the LSP abundance:

$$
\begin{aligned}
\Omega_{\mathrm{DM}} h^{2} & \simeq 0.12\left(\frac{1.6 \times 10^{4}}{\Delta}\right)\left(\frac{m_{\mathrm{LSP}}}{1 \mathrm{TeV}}\right)\left(\frac{\sqrt{\sum_{i}\left|\lambda_{6}^{i 0}\right|^{2}}}{0.0097}\right) \\
& =0.12\left(\frac{1.6 \times 10^{4}}{\Delta}\right)\left(\frac{m_{\mathrm{LSP}}}{1 \mathrm{TeV}}\right)\left(\frac{T_{\mathrm{RH}}}{1.6 \times 10^{13} \mathrm{GeV}}\right) .
\end{aligned}
$$

This non-thermal component of the LSP abundance should be combined with the component generated thermally to obtain the total LSP density. This is, however, reduced by the flaton entropy dilution factor $\Delta$ as discussed below, providing a direct relation between the LSP relic density and $\lambda_{6}$.

As we have just seen, the coupling $\lambda_{6}^{i 0}$ plays a crucial role in both reheating and the generation of gravitinos, and we discuss next a third role of this coupling - the generation of light neutrino masses ${ }^{146}$ As noted earlier, we adopt the basis where $\lambda_{2}^{i j}$ and $\mu^{a b}$ are real and diagonal. In this case, the diagonal components of $\lambda_{2}^{i j}$ is given by

$$
\lambda_{2} \simeq \frac{1}{\left\langle\bar{h}_{0}\right\rangle} \operatorname{diag}\left(m_{u}, m_{c}, m_{t}\right),
$$

and $\mu^{a b}$ is given in Eq. (246). Only three of the four singlets will contribute to the neutrino mass matrix and we take these to be $\phi_{a}, a=0,1,2$, including the inflaton. In what follows we express these matrices as $\lambda_{2}^{i j}=\lambda_{2}^{i} \delta^{i j}$ and $\mu^{a b}=\mu^{a} \delta^{a b} / 2$.

The relevant superpotential terms are

$$
W=\sum_{i=1}^{3} \lambda_{2}^{i} \nu_{i}^{c} L_{i} H_{d}+\frac{1}{2} \sum_{a=0}^{2} \mu^{a} \phi_{a}^{2}+\sum_{i, a} \lambda_{6}^{i a} \nu_{i}^{c} \nu_{\bar{H}}^{c} \phi_{a},
$$

where $\lambda_{6}^{i a}$ is a $3 \times 3$ complex matrix. The neutrino/singlet-fermion mass matrix can be written as

$$
\mathcal{L}_{\text {mass }}=-\frac{1}{2}\left(\begin{array}{lll}
\nu_{i} & \nu_{j}^{c} & \tilde{\phi}_{a}
\end{array}\right)\left(\begin{array}{ccc}
0 & \lambda_{2}^{i j}\left\langle\bar{h}_{0}\right\rangle & 0 \\
\lambda_{2}^{i j}\left\langle\bar{h}_{0}\right\rangle & 0 & \lambda_{6}^{j a}\left\langle\tilde{\nu}_{\bar{H}}^{c}\right\rangle \\
0 & \lambda_{6}^{j a}\left\langle\tilde{\nu}_{\bar{H}}^{c}\right\rangle & \mu^{a}
\end{array}\right)\left(\begin{array}{c}
\nu_{i} \\
\nu_{j}^{c} \\
\tilde{\phi}_{a}
\end{array}\right)+\text { h.c. },
$$

where $i, j=1,2,3, a=0,1,2$, and $\tilde{\phi}$ corresponds to the fermionic superpartner of the inflaton field $\phi$. (For more generic expressions, see Ref. 173). The mass matrix of the right-handed neutrinos is obtained from a first seesaw mechanism 174,175

$$
\left(m_{\nu^{c}}\right)_{i j}=\sum_{a=0,1,2} \frac{\lambda_{6}^{i a} \lambda_{6}^{j a}}{\mu^{a}}\left\langle\tilde{\nu}_{\bar{H}}^{c}\right\rangle^{2} .
$$

We can diagonalize the mass matrix 255) using a unitary matrix $U_{\nu^{c}}$ :

$$
m_{\nu^{c}}^{D}=U_{\nu^{c}}^{T} m_{\nu^{c}} U_{\nu^{c}} .
$$

Assuming $\left\langle\tilde{\nu}_{\bar{H}}^{c}\right\rangle=10^{16} \mathrm{GeV}$, the light neutrino mass matrix is then obtained through a second seesaw mechanism:

$$
\left(m_{\nu}\right)_{i j}=\sum_{k} \frac{\lambda_{2}^{i} \lambda_{2}^{j}\left(U_{\nu^{c}}\right)_{i k}\left(U_{\nu^{c}}\right)_{j k}\left\langle\bar{h}_{0}\right\rangle^{2}}{\left(m_{\nu^{c}}^{D}\right)_{k}} .
$$


This mass matrix is diagonalized by a unitary matrix $U_{\nu}$,

$$
m_{\nu}^{D}=U_{\nu}^{*} m_{\nu} U_{\nu}^{\dagger} .
$$

The Pontecorvo-Maki-Nakagawa-Sakata (PMNS) matrix in this model is given by 173

$$
U_{\mathrm{PMNS}}=U_{\ell}^{*} U_{\nu}^{\dagger}
$$

where $U_{\ell}$ is a unitary matrix that is used to diagonalize $\lambda_{3}$. We can always reproduce the measured PMNS matrix for arbitrary $U_{\nu}$ by choosing $U_{\ell}$ appropriately.

Given a matrix $\lambda_{6}^{i a}$, the eigenvalues of the $m_{\nu}$ and $m_{\nu^{c}}$ matrices, as well as the mixing matrices $U_{\nu^{c}}$ and $U_{\nu}$, are uniquely determined as functions of $\mu^{1}$ and $\mu^{2}$ via Eqs. 255 257. If we further assume that the field $\tilde{\phi}_{a}$ couples predominantly to the $i$-th generation neutrino, then we can write its mass approximately as

$$
m_{\nu_{i}} \simeq \frac{\mu^{a}\left(\lambda_{2}^{i}\left\langle\bar{h}_{0}\right\rangle\right)^{2}}{\left(\lambda_{6}^{i a}\left\langle\tilde{\nu}_{\bar{H}}^{c}\right\rangle\right)^{2}} \simeq \frac{\mu^{a} m_{u_{i}}^{2}}{\left(\lambda_{6}^{i a}\left\langle\tilde{\nu}_{\bar{H}}^{c}\right\rangle\right)^{2}} .
$$

This expression indicates that due to the hierarchical structure in $\lambda_{2}$ as shown in Eq. 2252, the mass eigenvalues of $m_{\nu}$ also become hierarchical unless there is a large hierarchy in $\mu^{a}$ or $\lambda_{6}$. In particular, the lightest mass eigenvalue tends to be much smaller than the other two masses, $m_{\nu_{1}} \ll m_{\nu_{2,3}}$. This means that for normal neutrino mass ordering (NO), $m_{\nu_{2}} \simeq \sqrt{\Delta m_{21}^{2}}=8.6 \times 10^{-3} \mathrm{eV}$ and $m_{\nu_{3}} \simeq \sqrt{\Delta m_{31}^{2}}=5.0 \times 10^{-2} \mathrm{eV}$, and for inverted neutrino mass ordering (IO), $m_{\nu_{2}} \simeq m_{\nu_{3}} \simeq \sqrt{\left|\Delta m_{32}\right|^{2}}=5.0 \times 10^{-2} \mathrm{eV}$, where the values of the squared mass differences, $\Delta m_{21}^{2} \equiv m_{2}^{2}-m_{1}^{2}$ and $\Delta m_{3 \ell}^{2} \equiv m_{3}^{2}-m_{\ell}^{2}(\ell=1,2)$ are given in the Table below, which we take from Ref. 176 .

Table 1. Input values for the squared mass differences of active neutrinos 176

\begin{tabular}{c|cc|cc}
\hline \hline & \multicolumn{2}{|c|}{ Normal Ordering } & \multicolumn{2}{c}{ Inverted Ordering } \\
& Best fit & $3 \sigma$ range & Best fit & $3 \sigma$ range \\
\hline$\Delta m_{21}^{2}\left[10^{-5} \mathrm{eV}^{2}\right]$ & 7.39 & $6.79-8.01$ & 7.39 & $6.79-8.01$ \\
$\Delta m_{3 \ell}^{2}\left[10^{-3} \mathrm{eV}^{2}\right]$ & 2.525 & $2.431-2.622$ & -2.512 & $-(2.413-2.606)$ \\
\hline \hline
\end{tabular}

The approximate formula 260 also shows that the couplings $\lambda_{6}^{i a}$ cannot be arbitrary small. For example, if we set $\mu^{0}=M=3 \times 10^{13} \mathrm{GeV}$ and $\left\langle\tilde{\nu}_{\bar{H}}^{c}\right\rangle=10^{16} \mathrm{GeV}$, $m_{\nu_{i}} \lesssim 0.1 \mathrm{eV}$ gives $\left|\lambda_{6}^{i 0}\right| \gtrsim 10^{-7}, 10^{-4}, 10^{-2}$ for $i=1,2,3$.

We recall that $\lambda_{6}^{i 0}$ also controls the reheating temperature and hence the thermal gravitino abundance, yielding the relic LSP abundance produced by gravitino decays shown in Eq. (251). In order to avoid overproduction of dark matter, we obtain the following upper limit on $\lambda_{6}^{i 0}$ from Eqs. 250 and 251)

$$
\sum_{i}\left|\lambda_{6}^{i 0}\right|^{2}<10^{-4}\left(\frac{\Delta}{1.6 \times 10^{4}}\right)^{2}\left(\frac{1 \mathrm{TeV}}{m_{\mathrm{LSP}}}\right)^{2} .
$$

We note that, if the thermal relic abundance of the LSP is negligibly small, the non-thermal LSP abundance from gravitino decay saturates the cosmological dark 
matter density for $\left|\lambda_{6}^{i 0}\right| \sim 10^{-2}$, at the border of the gravitino bound for $\Delta \sim 10^{4}$ and $m_{\mathrm{LSP}} \sim 1 \mathrm{TeV}$.

The right-handed neutrinos are massive only when $\langle\bar{H}\rangle \neq 0$. In the strong reheating scenario considered here, the masses of the right-handed neutrinos vanish and they are in thermal equilibrium immediately after reheating. They subsequently acquire masses at the GUT phase transition, and rapidly drop out of equilibrium. They decay non-thermally, $130[146] 147 \mid 171$ generating a lepton asymmetry as proposed in Ref. 156, which then generates a baryon asymmetry via the sphaleron process, $\frac{158}{15}$ yielding a net baryon number density

$$
\frac{n_{B}}{s}=-\frac{28}{79} \cdot \frac{135 \zeta(3)}{4 \pi^{4} g_{\mathrm{reh}} \Delta} \sum_{i=1,2,3} \epsilon_{i}
$$

where $130[173$

$$
\epsilon_{i}=\frac{1}{2 \pi} \frac{\sum_{j \neq i} \operatorname{Im}\left[\left(U_{\nu^{c}}^{\dagger}\left(\lambda_{2}^{D}\right)^{2} U_{\nu^{c}}\right)_{j i}^{2}\right]}{\left[U_{\nu^{c}}^{\dagger}\left(\lambda_{2}^{D}\right)^{2} U_{\nu^{c}}\right]_{i i}} g\left(\frac{m_{\nu_{j}^{c}}^{2}}{m_{\nu_{i}^{c}}^{2}}\right)
$$

$\operatorname{with}^{177}$

$$
g(x) \equiv-\sqrt{x}\left[\frac{2}{x-1}+\ln \left(\frac{1+x}{x}\right)\right]
$$

Thus we see that $\lambda_{6}$ in fact determines not only the reheating temperature, the nonthermal component of cold dark matter, and neutrino masses, but also the baryon asymmetry of the Universe.

The estimate based on Eq. (260) is inaccurate if the inflaton field couples to several generations or if the mixing matrices $U_{\nu^{c}}$ and $U_{\nu}$ have sizable off-diagonal components. In order to consider such cases, we have investigated numerically the effect of the $\lambda_{6}$ coupling matrix on neutrino masses, the reheating temperature, the non-thermal component of the LSP density, and the baryon asymmetry of the Universe, by performing a scan over the $\lambda_{6}$ parameter space ${ }^{[130}$ We write $\lambda_{6}$ in the form $146 \mid 147$

$$
\lambda_{6}=r_{6} M_{6},
$$

where $r_{6}$ is a real constant, which plays a role of a scale factor, and $M_{6}$ is a generic complex $3 \times 3$ matrix. We scan $r_{6}$ with a logarithmic distribution over the range $\left(10^{-4}, 1\right)$ choosing a total of 2000 values. For each value of $r_{6}$, we generate a random complex $3 \times 3$ matrix $M_{6}$ with each component taking a value of $\mathcal{O}(1)$.

We obtain the eigenvalues of the $m_{\nu}$ and $m_{\nu^{c}}$ matrices as functions of $\mu^{1}$ and $\mu^{2}$ for each choice of the $3 \times 3$ matrix $\lambda_{6}$, The two $\mu$ parameters are then fixed by reproducing within experimental uncertainties the measured values of the squared mass differences, $\Delta m_{21}^{2} \equiv m_{2}^{2}-m_{1}^{2}$ and $\Delta m_{3 \ell}^{2} \equiv m_{3}^{2}-m_{\ell}^{2}$, where $\ell=1(2)$ in the case of NO (IO). We use the results of Ref. 176 as experimental input, which we summarize in Table 1. Out of the $4 \times 10^{6}$ model $\lambda_{6}$ matrices we generate for each 
ordering of the neutrino masses, we find 9839 and 730 acceptable matrix choices in the NO and IO cases, respectively. This difference may suggest a mild preference for the NO case in our model.

Fig. 15 displays histograms of the reheating temperature $T_{\mathrm{RH}}$ in the $\mathrm{NO}$ and IO scenarios, using orange shading and a dashed blue line, respectively. In both cases, all parameter points have $T_{\mathrm{RH}} \gg\left(m_{\Phi} M_{\mathrm{GUT}}\right)^{1 / 2} \simeq 10^{10} \mathrm{GeV}$, so the strong reheating condition is satisfied for $\Lambda_{c} \gtrsim 2.3 \times 10^{10} \mathrm{GeV} ! 130$ We also see that values of $T_{\mathrm{RH}} \sim 10^{12} \mathrm{GeV}$ are favoured in both the NO and IO cases, with much larger values $\lesssim 10^{15} \mathrm{GeV}$ also being possible in the NO scenario.

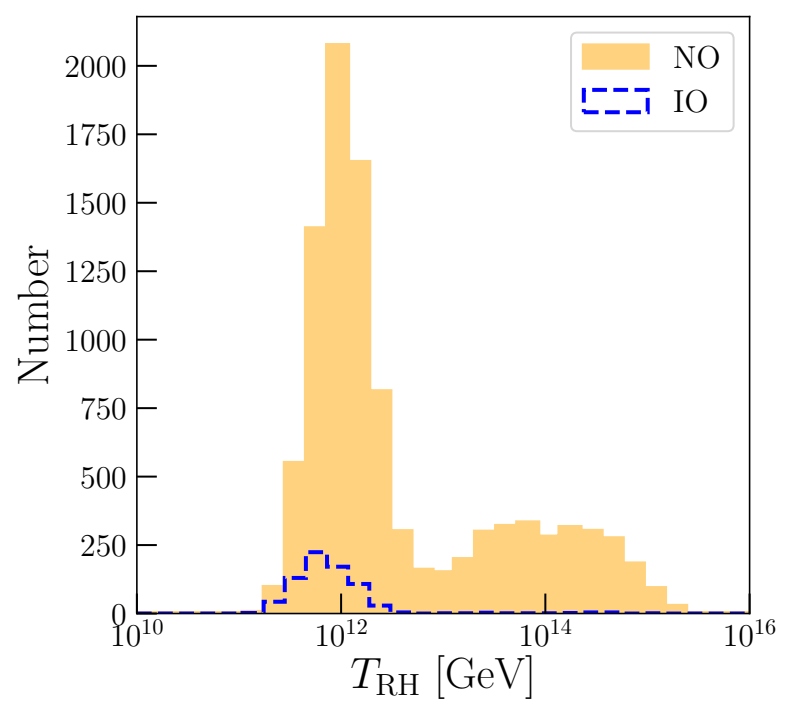

Fig. 15. Histograms of the values of $T_{\mathrm{RH}}$ resulting from the numerical scan of $\lambda_{6}$ for the NO and IO cases (orange shading and dashed blue line, respectively).

The distributions of the non-thermal dark matter density produced by gravitino decays in the NO and IO solutions for $\lambda_{6}$ is shown in Fig. 16, assuming that the flaton entropy dilution factor estimated in 245 has the value $\Delta=10^{4}$. Many parameter sets yield $\Omega_{\mathrm{DM}} h^{2} \simeq 10^{-1}$, consistent with the density of dark matter measured by the Planck collaboration, $\Omega_{\mathrm{DM}} h^{2}=0.12 \frac{10}{10}$ which is shown as the black line in Fig. 16 for $m_{\mathrm{LSP}}=10 \mathrm{TeV}$, corresponding to $T_{\mathrm{RH}} \simeq 10^{12} \mathrm{GeV}$, as seen in Eq. 251). Some solutions overproduce dark matter for $m_{\mathrm{LSP}}=10 \mathrm{TeV}$, yielding $\Omega_{\mathrm{DM}} h^{2} \lesssim 10$, corresponding to $T_{\mathrm{RH}} \simeq 10^{14} \mathrm{GeV}$. In such cases, either smaller values of $m_{\mathrm{LSP}}$ and/or larger entropy factors $\Delta$ would be required for consistency with observation.

In Fig. 17, we show histograms of $n_{B} / s$ assuming an entropy factor $\Delta=10^{4}$. The observed value of the baryon asymmetry, $n_{B} / s=0.87 \times 10^{-10,10}$ is shown as the vertical solid line in Fig. 17, and can easily be explained in our scenario. A value 


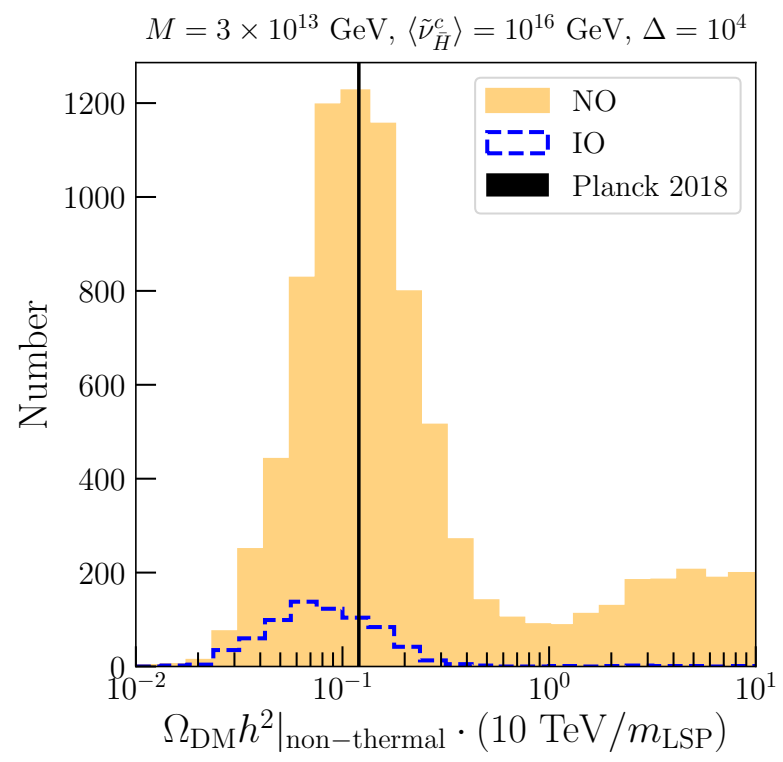

Fig. 16. Histograms of the values of $\Omega_{\mathrm{DM}} h^{2}$ found in the numerical scan of $\lambda_{6}$ for the $N O$ and IO cases (orange shading and blue dashed line, respectively) with $\Delta=10^{4}$. The vertical black line shows the Planck 2018 value of the dark matter density: $\Omega_{\mathrm{DM}} h^{2}=0.12 ! 10$

of $\Delta$ much more than two orders of magnitude larger would be unlikely to yield an acceptable value of $n_{B} / s$.

The non-thermal contribution to the LSP abundance due to gravitino decay assuming $m_{\mathrm{LSP}}=10 \mathrm{TeV}$ is plotted in Fig. 18 against the value of $n_{B} / s$ for the same parameter point, assuming that a factor $\Delta=10^{4}$ of entropy is generated. The horizontal green and vertical black lines show the measured values of the dark matter abundance $\Omega_{\mathrm{DM}} h^{2}=0.12$ and the baryon asymmetry ${ }^{10}$ respectively. We see that for most of the points $\Omega_{\mathrm{DM}} h^{2} \gtrsim \mathcal{O}\left(10^{-2}\right)$ and $n_{B} / s \lesssim \mathcal{O}\left(10^{-7}\right)$. There are many points where the non-thermal component of the LSP abundance from gravitino decays provides all the dark matter density, $\Omega_{\mathrm{DM}} h^{2} \simeq 0.12$, and $n_{B} / s \simeq 0.87 \times 10^{-10}$.

The amount of entropy production discussed above has important consequences for the low-energy supersymmetric parameter space. In many models, $\frac{34}{39}$ all the soft scalar masses are assumed to have the same value $m_{0}$ at some high-energy input scale, $M_{i n}$, which may be the same as $M_{\mathrm{GUT}}$, as in the CMSSM, and similarly for the gaugino masses, $m_{1 / 2}$, and the trilinear soft supersymmetry-breaking terms, $A_{0}$. The non-observation of supersymmetry at the $\mathrm{LHC}^{23}$ indicates that sparticle masses must be at or above the $\mathrm{TeV}$ scale, in which case one would normally expect the relic LSP density following thermal freeze-out to exceed generically the cold dark matter density measured by Planck, $\Omega h^{2} \simeq 0.12 \cdot \frac{10}{10}$ However, this problem can be avoided in the presence of particular relations between the masses of the LSP and some other sparticles. 


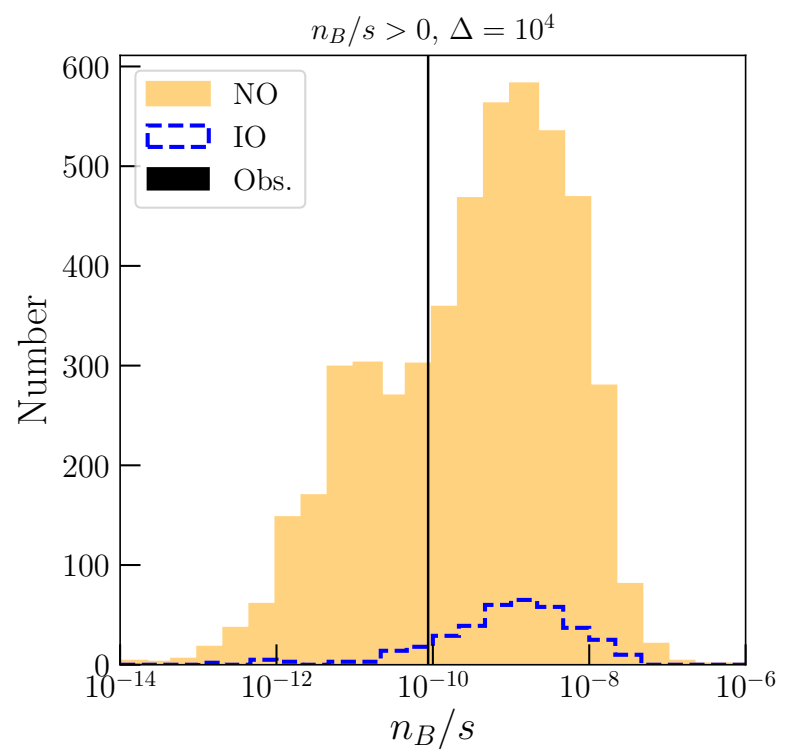

Fig. 17. Histograms of values of $n_{B} / s$ in the NO and IO scenarios (orange shading and blue dashed, respectively) assuming an entropy factor $\Delta=10^{4}$. The vertical black solid line shows the observed value.

Consider, for example, a bino LSP $\chi$ that annihilates to SM fermions via $t$ channel sfermion exchange. In the limit $m_{\tilde{f}}>m_{\chi} \gg m_{f}$, where $m_{\tilde{f}}$ is a common sfermion mass and $m_{\chi}$ is the bino mass, the $p$-wave annihilation cross section may be approximated by 178

$$
\langle\sigma v\rangle \simeq \frac{g_{1}^{4}}{32 \pi} \sum_{f}\left(Y_{L f}^{4}+Y_{R f}^{4}\right) \frac{m_{\chi}^{2}}{m_{\tilde{f}}^{4}} x,
$$

where $g_{1}$ is the $\mathrm{U}(1)_{Y}$ gauge coupling, $Y_{L, R}$ are the hypercharges of the left- and right-handed fermions, respectively, and $x=T_{f} / m_{\chi} \approx 1 / 20$ is the ratio of the annihilation freeze-out temperature to the bino mass. In such a case, the relic density is given approximately by $47[179$

$$
\Omega_{\chi} h^{2} \approx 1.9 \times 10^{-11}\left(\frac{T_{\chi}}{T_{\gamma}}\right)^{3} \sqrt{g_{f}}\left(\frac{\mathrm{GeV}^{-2}}{\frac{1}{2}\langle\sigma v\rangle x}\right),
$$

where $g_{f}$ is the number of relativistic degrees of freedom at freeze-out and the factor $\left(T_{\chi} / T_{\gamma}\right)^{3}$ is due to the dilution of neutralinos between freeze-out and today $47 \mid 180$ If $m_{\chi} \sim 100 \mathrm{GeV}$ and $m_{\tilde{f}} \sim 350 \mathrm{GeV}$, one finds $\Omega_{\chi} h^{2} \sim 0.1$. However, 267 shows that $\Omega_{\chi} h^{2} \propto m_{\tilde{f}}^{4} / m_{\chi}^{2}$, so that values of sparticle masses a factor of 100 larger, i.e., $m_{\chi} \sim 10 \mathrm{TeV}$ and $m_{\tilde{f}} \sim 35 \mathrm{TeV}$, would lead to $\Omega_{\chi} h^{2}=\mathcal{O}\left(10^{3}\right)$.

This argument may be evaded if the LSP and the next-to-lightest supersymmetric particle (NLSP) are very similar in mass, in which case coannihilations between 


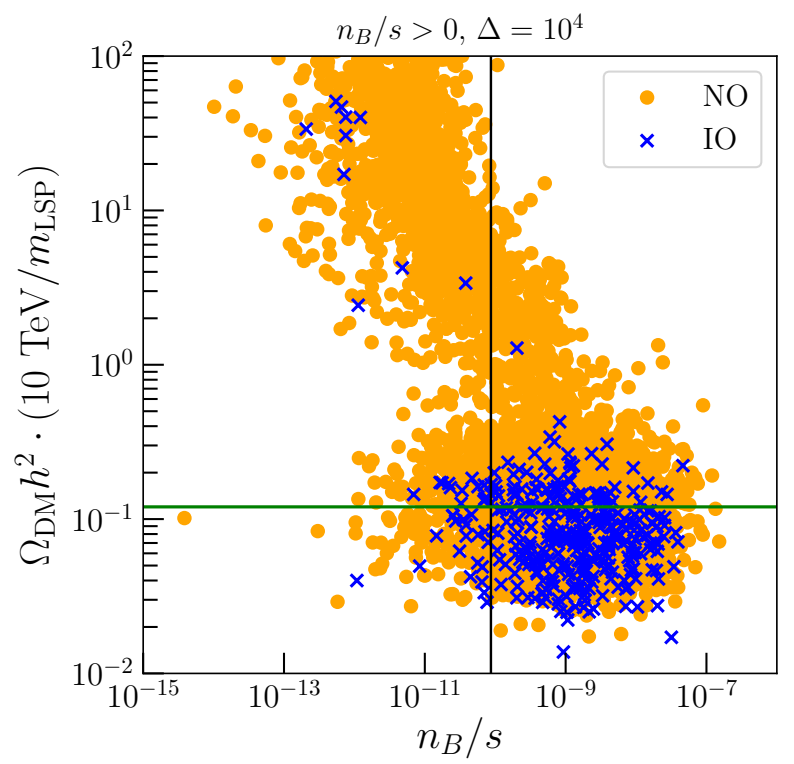

Fig. 18. Scatter plot of $n_{B} / s$ vs the non-thermal contribution to the LSP abundance, assuming $m_{\mathrm{LSP}}=10 \mathrm{TeV}$ and $\Delta=10^{4}$, with the observed values shown as the horizontal green and vertical black lines, respectively.

them can reduce the relic density ${ }^{181}$ Examples of possible nearly-degenerate NL-

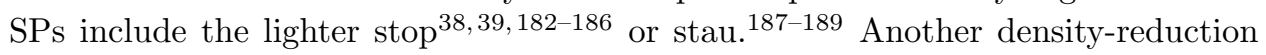
mechanism comes into play when the LSP mass is very close to half the mass of the heavy MSSM Higgs scalar and/or pseudoscalar, whose $s$-channel exchanges lead to rapid annihilations. $\frac{190}{10}$ Yet another possibility is that $m_{0} \gg m_{1 / 2}$ and $A_{0}$ is small. In this case, the Higgs mixing parameter $\mu$ is also small, the LSP resembles a Higgsino, and annihilations into $W^{ \pm}$and $Z^{0}$ bosons become important $\frac{191}{1}$ This again requires quite a finely-tuned relationship between $m_{1 / 2}$ and $m_{0}$ for any given values of $A_{0}$ and $\tan \beta$.

However, the landscape of allowed models changes greatly in the presence of late-time entropy production. Specifically, a factor $\Delta=10^{4}$ of entropy production in flaton decay would imply an increase in the preferred relic density at freeze-out by $10^{4}$, corresponding to generic sparticle masses of order $10 \mathrm{TeV}$, as can be deduced from Eqs. 266 and 267). Bearing in mind that the strong reheating scenario prefers $\Delta=10^{4}$ for other reasons, one may regard a supersymmetry-breaking scale of $\mathcal{O}(10) \mathrm{TeV}$ as a prediction of the scenario. We can estimate the relic density in Eq. (267) as

$$
\Omega_{\chi} h^{2} \simeq 10^{-7} \mathrm{GeV}^{-2} \Delta^{-1} \frac{m_{\tilde{f}}^{4}}{m_{\chi}^{2}} \sim 10^{3} \Delta^{-1}\left(\frac{m_{\tilde{f}}}{30 \mathrm{TeV}}\right)^{4}\left(\frac{10 \mathrm{TeV}}{m_{\chi}}\right)^{2},
$$


where the entropy release is approximately 130

$$
\Delta \sim 10^{4}\left(\frac{30 \mathrm{TeV}}{m_{\tilde{f}}}\right)^{1 / 2}
$$

yielding

$$
\Omega_{\chi} h^{2} \sim 10^{-1}\left(\frac{m_{\tilde{f}}}{30 \mathrm{TeV}}\right)^{9 / 2}\left(\frac{10 \mathrm{TeV}}{m_{\chi}}\right)^{2}
$$

where all the relevant couplings are assumed to be $\mathcal{O}(1)$.

One should also take into account the condition for successful BBN that the reheating temperature after flaton decay! 130

$$
T_{\mathrm{RH}}^{\prime} \sim 10^{-3}\left(\frac{m_{\tilde{f}}^{3} M_{P}}{M_{\mathrm{GUT}}^{2}}\right)^{1 / 2} \sim 1 \mathrm{MeV}\left(\frac{m_{\tilde{f}}}{30 \mathrm{TeV}}\right)^{3 / 2},
$$

be $\gtrsim 1 \mathrm{MeV}$, ensuring that the universe is radiation-dominated during BBN. Combining Eqs. 270 and 271), we find

$$
\Omega_{\chi} h^{2} \sim 0.1\left(\frac{T_{\mathrm{RH}}^{\prime}}{1 \mathrm{MeV}}\right)^{3}\left(\frac{10 \mathrm{TeV}}{m_{\chi}}\right)^{2} .
$$

We find that the reheating temperature in Eq. (271) is $\gtrsim 1 \mathrm{MeV}$, as needed for $\mathrm{BBN}$, for $m_{\tilde{f}} \gtrsim \mathcal{O}(10) \mathrm{TeV}$. On the other hand, imposing the cosmological limit $\Omega_{\chi} h^{2} \leq 0.12$ in Eq. 268 requires $m_{\tilde{f}} \lesssim \mathcal{O}(10) \mathrm{TeV}$. Thus, in this model consistency between these constraints determines the supersymmetry breaking scale to be $\mathcal{O}(10)$ $\mathrm{TeV}$. This prediction is consistent with the non-observation of SUSY signals at LHC so far, while offering hope for detection at a future 100-TeV proton-proton collider.

To conclude this discussion, we show illustrative results from one example of a super-GUT $122|192| 194$ CMSSM based on flipped SU(5) as originally considered in Ref.194. In super-GUT models, the universality of the soft supersymmetry-breaking parameters occurs at some scale $M_{i n}$ above the GUT scale. In the flipped superGUT plane shown in Fig. 19, we take $A_{0} / m_{0}=0, \tan \beta=10, M_{i n}=M_{P}$ and $\mu>0$, and the flipped SU(5) couplings are chosen as $\boldsymbol{\lambda}=\left(\lambda_{4}, \lambda_{5}\right)=(0.3,0.3)$. Across the plane, in the absence of entropy generation the relic density is significantly larger than its observationally-determined value. Indeed, $\Omega_{\chi} h^{2}$ easily reaches $\mathcal{O}(1000)$ when the measured value of the lightest Higgs boson mass $m_{h}=125 \mathrm{GeV}$. We see from this Figure that an entropy factor $\Delta=\mathcal{O}\left(10^{4}\right)$, as suggested above, would reduce the relic LSP density to an allowed value over a large range of the soft supersymmetry-breaking gaugino mass $m_{1 / 2} \sim$ a few $\times 10^{3} \mathrm{GeV}$ (corresponding to strongly-interacting sparticle masses $\sim 10 \mathrm{TeV}$, beyond the reach of the LHC) that are compatible with the measured value of $m_{h}$, within the expected theoretical error of $\pm 3 \mathrm{GeV}$, for values of the soft supersymmetry-breaking scalar mass $m_{0} \lesssim 1 \mathrm{TeV}$. 


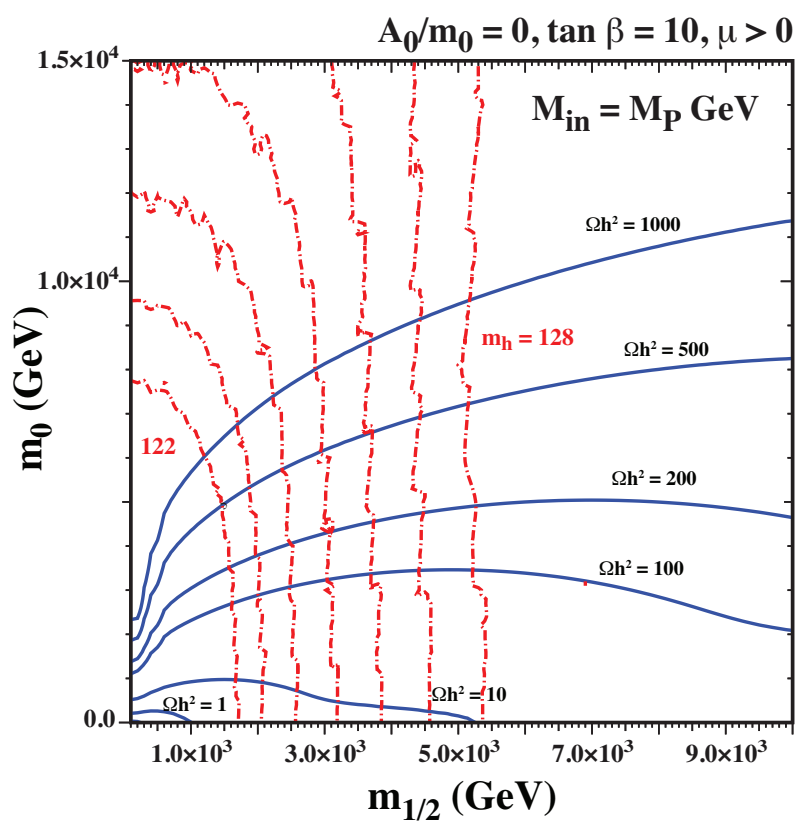

Fig. 19. $A\left(m_{1 / 2}, m_{0}\right)$ plane in the flipped super-GUT model with $M_{i n}=M_{P}, \tan \beta=10, \mu>0$, $A_{0}=0, \boldsymbol{\lambda}=(0.3,0.3)$. The red dot-dashed lines are contours of $m_{h}$ calculated using FeynHiggs, 195 and the solid blue lines are contours of $\Omega_{\chi} h^{2}$ in the absence of subsequent entropy generation. This figure is not sensitive to the choice of $\lambda_{6}$.

\subsection{Nucleon decay}

In the models discussed in the previous Sections, nucleon decay occurs through the exchange of GUT-scale particles. In particular, the standard and flipped SU(5) GUT models described in Sections 12.1 and 12.3 respectively, have characteristic predictions for nucleon decay branching ratios because of the minimality of these models $\frac{148}{1 n}$ In this Section, we briefly review these features and discuss the possibility of distinguishing these models in future proton decay experiments.

In the minimal standard SU(5) model in Section 12.1, $p \rightarrow K^{+} \bar{\nu}$ induced by the exchange of color-triplet Higgs fields is the dominant decay mode ${ }^{196}$ In fact, the lifetime of this decay mode turns out to be too short if supersymmetry lies around the $\mathrm{TeV}$ scale $197 \mid 198$ though this problem is alleviated if sparticle have larger masses $36|37| 127|153|[199]$ The $p \rightarrow K^{+} \bar{\nu}$ mode in constrained supersymmetric models has recently been investigated in detail in Ref. 153, it is found that the predicted rate of this decay mode can exceed the current limit imposed by SuperKamiokande, $\tau\left(p \rightarrow K^{+} \bar{\nu}\right)>6.6 \times 10^{33}$ years, ${ }^{200}$ in a range of the parameter space that is consistent with the measured value of the Higgs boson mass, and that it is within the reach of future proton decay experiments, such as JUNO, 201 DUNE, 202 and Hyper-Kamiokande ${ }^{203}$ If sparticle masses are as large as $\gtrsim 100 \mathrm{TeV}$, as in PGM,, 126$] 127$ the dominant decay mode becomes $p \rightarrow e^{+} \pi^{0}$ induced by the exchange 
of the $\mathrm{SU}(5)$ gauge bosons $\frac{127}{127}$ which may also be probed in Hyper-Kamiokande.$^{203}$ The rates of other decay modes, such as $p \rightarrow \mu^{+} \pi^{0}$. 1 are quite suppressed unless there is sizable flavor violation in sfermion mass matrices ${ }^{199}$ and likely to be beyond the reach of the future proton decay experiments.

In summary, the prediction for proton decay in the minimal standard $\mathrm{SU}(5)$ model in Section 12.1 is as follows: i) $p \rightarrow K^{+} \bar{\nu}$ is the dominant decay mode for relatively light supersymmetric mass spectra; ii) $p \rightarrow e^{+} \pi^{0}$ is the dominant decay mode for heavier sparticle masses; iii) only these two decay modes are within the reach of future proton decay experiments.

Nucleon decay predictions in the flipped SU(5) model discussed in Section 12.3 are quite different. A detailed comparison of the nucleon decay branching fractions in the flipped and unflipped SU(5) GUTs is given in Ref. 148, The decay modes that are most promising for detection in future experiments are: i) $p \rightarrow K^{+} \bar{\nu}$ and ii) $\Gamma\left(p \rightarrow \mu^{+} \pi^{0}\right) / \Gamma\left(p \rightarrow e^{+} \pi^{0}\right)$.

We note first that in the flipped SU(5) model the color-triplet Higgs exchange process is suppressed due to the absence of a supersymmetric mass term for $H$ and $\bar{H}$. In addition, it is found that the GUT gauge boson exchange process does not induce $p \rightarrow K^{+} \bar{\nu}$ either ${ }^{173}$ As a result, the branching ratio for decay into $p \rightarrow K^{+} \bar{\nu}$ is negligible in flipped $\mathrm{SU}(5)$, in contrast to standard $\mathrm{SU}(5)$, where it is expected to be the dominant decay mode, as discussed above.

Secondly, the ratio of the decay rates of $p \rightarrow e^{+} \pi^{0}$ and $p \rightarrow \mu^{+} \pi^{0}$ in flipped $\mathrm{SU}(5)$ is found to be 173

$$
\frac{\Gamma\left(p \rightarrow \mu^{+} \pi^{0}\right)}{\Gamma\left(p \rightarrow e^{+} \pi^{0}\right)} \simeq \frac{\left|\left(U_{\ell}\right)_{21}\right|^{2}}{\left|\left(U_{\ell}\right)_{11}\right|^{2}}
$$

where $U_{\ell}$ is a unitary matrix that is used to diagonalize $\lambda_{3}$, which can be obtained from $U_{\nu}$ and the PMNS matrix using Eq. (259). We show in Fig. 20 histograms of the ratio $\Gamma\left(p \rightarrow \pi^{0} \mu^{+}\right) / \Gamma\left(p \rightarrow \pi^{0} e^{+}\right)$in the normal-ordered (NO) and inverse-ordered (IO) neutrino mass scenarios in blue and green, respectively. The vertical black solid line represents the value predicted in standard SU(5). The flipped SU(5) model predicts this ratio to be $\sim 0.10$ and $\sim 23$ for the NO and IO cases, respectively, which is much larger than the standard SU(5) prediction. Hyper-Kamiokande ${ }^{203}$ is expected to improve by an order of magnitude the present experimental sensitivity to both of these decay modes, which may provide an opportunity to test these predictions.

\section{Summary}

The road to building a no-scale model of inflation has been a long and winding one. We have argued that inflation must involve Planck-scale physics,$\frac{5}{5}$ and that inflation cries out for supersymmetry $\stackrel{8}{8}$ As a result, inflationary models should be built

${ }^{\text {i The ratio } \Gamma\left(p \rightarrow \mu^{+} \pi^{0}\right) / \Gamma\left(p \rightarrow e^{+} \pi^{0}\right)}$ is predicted to be $\simeq 0.008$ in minimal standard $\mathrm{SU}(5) ! 148$ 


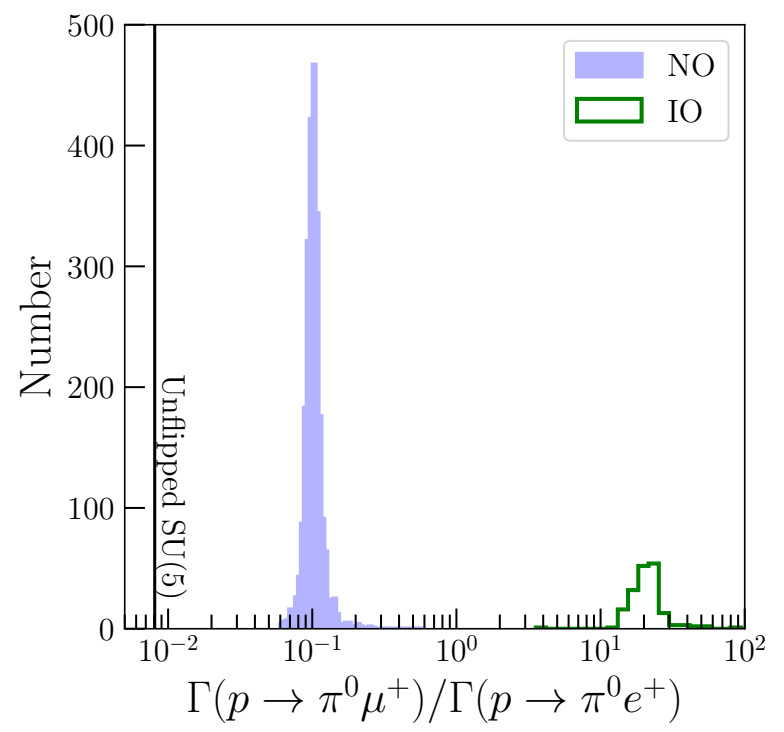

Fig. 20. Histograms of $\Gamma\left(p \rightarrow \pi^{0} \mu^{+}\right) / \Gamma\left(p \rightarrow \pi^{0} e^{+}\right)$in the flipped SU(5) GUT model for the cases of normal-ordered (NO) and inverse-ordered (IO) neutrino mass spectra shown in blue and green, respectively. The vertical line corresponds to the unflipped SU(5) prediction.

from supergravity, in particular no-scale supergravity, 15 which yields naturally a semi-positive potential with flat directions and no AdS holes. Moreover, no-scale supergravity emerges naturally in the low-energy limit of string theory ${ }^{19}$ However, supergravity, even no-scale supergravity, is not sufficient as a framework to specify the model of inflation. As is usually the case, theory must rely on experiment to make progress. Measurements of the CMB anisotropy spectrum, (relatively) quickly ruled out large numbers of inflationary models, including some very simple models described in Section 3.2 based on minimal and no-scale supergravity (from measurements of $n_{s}$ ), as well as models based on single polynomial potentials (from upper limits to $r$ ).

Curiously, the models suggesting a singularity-free Universe proposed by Starobinsky ${ }^{\sqrt{3}}$ are equivalent up to a conformal transformation ${ }^{4}$ to single-field models of inflation with a plateau-like potential - see Eq. 40 and Fig. 4- which are in excellent agreement with current CMB measurements. A significant theoretical advance was made when it was realized that, starting with a very simple Wess-Zumino form of superpotential (44), the Starobinsky potential could be derived from no-scale supergravity, 20 as we have discussed in Section 5 . It was further realized that the superpotential yielding the Starobinsky potential was not unique ${ }^{82}$ Indeed, it had been shown previously ${ }^{99}$ that $R+R^{2}$ gravity was equivalent to a different no-scale model with the superpotential given in Eq. (136). As described in Section 9 , these models are all related by the underlying $\mathrm{SU}(2,1) / \mathrm{SU}(2) \times \mathrm{U}(1)$ no-scale symmetry $[98$ 
The parallels between the conformal transformation in supergravity, and higherderivative gravity were reviewed ${ }^{84}$ in Sections 6 and 7 . There we saw that the scale invariance of an $R^{2}$ theory of gravity is directly related to the choice of the conformal function, $\Phi$, made in Eq. 64, leading to a Kähler potential with a noscale symmetry. Since $R^{2}$ gravity is equivalent to a de Sitter space-time $\frac{85}{15}$ once the connection between $R^{2}$ gravity and no-scale supergravity is made, it is natural to consider the construction of Minkowski, de Sitter, and anti-de Sitter solutions in no-scale supergravity ${ }^{93} \mid 93$ This connection was reviewed in Section 8 .

Of course, the inflationary sector cannot be totally isolated from the matter sector, as reheating and a graceful exit from inflation are necessary. As a consequence, inflation can not be divorced from the mechanism of supersymmetry breaking $104 \mid 115$ No-scale inflation and phenomenology were discussed in Section 11. In particular,

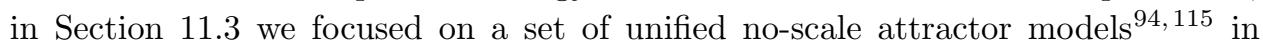
which inflation, supersymmetry breaking, and dark energy can all be explained in a simple and compact framework given by the superpotential in Eq. 157). In these models, the inflaton mass, $M$, determines the scale of supersymmetry breaking, with $m_{3 / 2} \sim M^{3} / M_{P}^{2}$. We discussed the mechanisms for reheating and the relation to the number of e-folds of inflation in Sections 11.5 and 11.6 respectively.

Finally, in Section 12, we showed how to embed no-scale models of inflation in various GUTs. We considered in turn an SU(5) GUT model, an SO(10) GUT model, 144 and a flipped $\mathrm{SU}(5) \times \mathrm{U}(1)$ model 130,145

We summarize our approach to building a no-scale inflationary cosmology in Fig. 21. The theory is based on no-scale supergravity (left orange box) which is derived from the superstring (green box) $\frac{19}{19}$ The no-scale Starobinsky-like model of inflation (grey box) makes successful predictions for the tensor-to-scalar perturbation ratio and the tilt in the scalar perturbation spectrum (upper left pink boxes).

As we have emphasized in Section 12.3, our preferred field-theoretical framework is the flipped $\mathrm{SU}(5) \times \mathrm{U}(1)$ GUT (right orange box), which may be derived from weakly-coupled heterotic string theory ${ }^{21]}$ In this scenario there is one particular

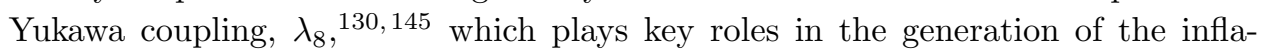
tionary potential. Another coupling, $\lambda_{6}$, plays a crucial role $\underline{146}[147$ in inflaton decay and the reheating process, in leptogenesis, and in generating via a double-seesaw mechanism neutrino masses (right pink box) that are compatible with oscillation measurements and cosmological limits. As we have discussed in Section 12.4, this model's predictions for nucleon decay are different from those of conventional SU(5) (which cannot be derived from weakly-coupled string theory), and may be accessible in the next round of neutrino oscillation experiments. 148

In this scenario, the decay of the inflaton is thought to have caused strong reheating of the Universe followed by the GUT phase transition (central grey box) associated with a flaton field, which generated a factor $\Delta=\mathcal{O}\left(10^{4}\right)$ of entropy (lowest blue box). This diluted the baryon asymmetry $n_{B} / s$ generated by leptogenesis to a value compatible with cosmological measurements (right pink box), and also diluted the gravitino abundance (left grey box) so that the density of cold 


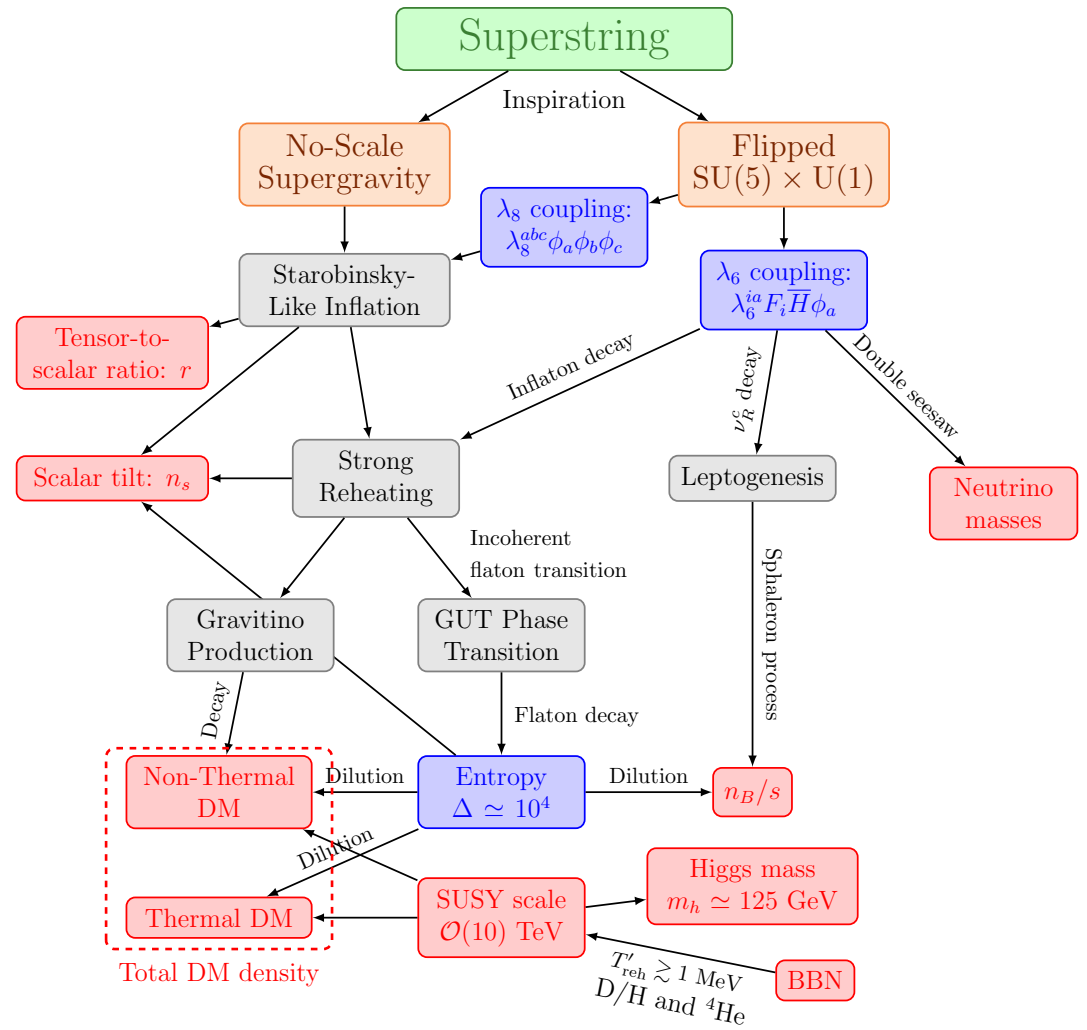

Fig. 21. The general structure of our preferred scenario for no-scale inflation and particle cosmology 147

dark matter produced non-thermally by gravitino decay is also compatible with cosmological measurements as well that produced thermally (left pink boxes) with a supersymmetry-breaking scale that is $\mathcal{O}(10) \mathrm{TeV}$ (lower pink box) ${ }^{147}$ Although placing sparticles beyond the reach of the LHC, this scale is comfortably compatible with the measured value of the Higgs mass $m_{h} \simeq 125 \mathrm{GeV}$, as well as the baryonto-entropy ratio indicated by the success of conventional Big Bang Nucleosynthesis calculations.

The scenario depicted in Fig. 21 illustrates how no-scale supergravity inflation provides a possible bridge between string theory in the ultraviolet limit and the Standard Model at $\mathrm{TeV}$ energies, and in particular a framework for a flipped model of almost everything below the Planck scale. 


\section{Acknowledgments}

The work of J.E. was supported partly by the United Kingdom STFC Grant ST/P000258/1 and partly by the Estonian Research Council via a Mobilitas Pluss grant. The work of D.V.N. was supported partly by the DOE grant DE-FG0213ER42020 and partly by the Alexander S. Onassis Public Benefit Foundation. The work of K.A.O. and S.V. was supported partly by the DOE grant DE-SC0011842 at the University of Minnesota and acknowledges support by the Director, Office of Science, Office of High Energy Physics of the U.S. Department of Energy under the Contract No. DE-AC02-05CH11231. The work of N.N. was supported by the Grantin-Aid for Scientific Research B (No.20H01897), Young Scientists B (No.17K14270), and Innovative Areas (No.18H05542). The work of M.A.G.G. was supported by the Spanish Agencia Estatal de Investigación through the grants FPA2015-65929-P (MINECO/FEDER, UE), PGC2018095161-B-I00, IFT Centro de Excelencia Severo Ochoa SEV-2016-0597, and Red Consolider MultiDark FPA2017-90566-REDC.

\section{References}

1. A. H. Guth, Phys. Rev. D 23 (1981) 347.

2. K. A. Olive, Phys. Rept. 190 (1990) 307; A. D. Linde, Particle Physics and Inflationary Cosmology (Harwood, Chur, Switzerland, 1990); D. H. Lyth and A. Riotto, Phys. Rep. 314 (1999) 1 arXiv:hep-ph/9807278]; J. Martin, C. Ringeval and V. Vennin, Phys. Dark Univ. 5-6, 75-235 (2014) arXiv:1303.3787 [astroph.CO]]; J. Martin, C. Ringeval, R. Trotta and V. Vennin, JCAP 1403 (2014) 039 arXiv:1312.3529 [astro-ph.CO]]; J. Martin, Astrophys. Space Sci. Proc. 45, 41 (2016) arXiv:1502.05733 [astro-ph.CO]].

3. A. A. Starobinsky, Phys. Lett. B 91, 99 (1980).

4. K. S. Stelle, Gen. Rel. Grav. 9 (1978) 353; B. Whitt, Phys. Lett. 145B (1984) 176.

5. J. R. Ellis, D. V. Nanopoulos, K. A. Olive and K. Tamvakis, Nucl. Phys. B 221 (1983) 52 .

6. V. F. Mukhanov and G. V. Chibisov, JETP Lett. 33, 532 (1981) [Pisma Zh. Eksp. Teor. Fiz. 33, 549 (1981)]; A. A. Starobinsky, Sov. Astron. Lett. 9, 302 (1983).

7. W.H. Press, Phys. Scr. 21 (1980) 702; S.W. Hawking, Phys. Lett. 115B (1982) 295; A.A. Starobinsky, Phys. Lett. 117B (1982) 175; A.H. Guth and S.Y. Pi, Phys. Rev. Lett. 49 (1982) 1110; J.M. Bardeen, P.J. Steinhardt and M.S. Turner, Phys. Rev. D28 (1983) 679.

8. J. R. Ellis, D. V. Nanopoulos, K. A. Olive and K. Tamvakis, Phys. Lett. 118B (1982) 335; K. Nakayama and F. Takahashi, JCAP 1110, 033 (2011) arXiv:1108.0070 [hep$\mathrm{ph}]]$.

9. J. R. Ellis, D. V. Nanopoulos, K. A. Olive and K. Tamvakis, Phys. Lett. B 120 (1983) 331.

10. N. Aghanim et al. [Planck Collaboration], arXiv:1807.06209 [astro-ph.CO]; Y. Akrami et al. [Planck Collaboration], arXiv:1807.06211][astro-ph.CO].

11. P. A. R. Ade et al. [BICEP2 and Keck Array Collaborations], Phys. Rev. Lett. 121, 221301 (2018) arXiv:1810.05216 [astro-ph.CO]].

12. E. Cremmer, B. Julia, J. Scherk, P. van Nieuwenhuizen, S. Ferrara and L. Girardello, Phys. Lett. B 79, 231-234 (1978); E. Cremmer, B. Julia, J. Scherk, S. Ferrara, L. Girardello and P. van Nieuwenhuizen, Nucl. Phys. B 147, 105 (1979); E. Cremmer, S. Ferrara, L. Girardello and A. Van Proeyen, Phys. Lett. B 116, 231-237 (1982); 
E. Cremmer, S. Ferrara, L. Girardello and A. Van Proeyen, Nucl. Phys. B 212, 413 (1983).

13. H. P. Nilles, Phys. Rept. 110 (1984) 1.

14. E. J. Copeland, A. R. Liddle, D. H. Lyth, E. D. Stewart and D. Wands, Phys. Rev. D 49, 6410 (1994) astro-ph/9401011] E. D. Stewart, Phys. Rev. D 51, 6847 (1995) hep-ph/9405389.

15. E. Cremmer, S. Ferrara, C. Kounnas and D. V. Nanopoulos, Phys. Lett. B 133 (1983) 61; J. R. Ellis, A. B. Lahanas, D. V. Nanopoulos and K. Tamvakis, Phys. Lett. B 134 (1984) 429;

16. J. R. Ellis, C. Kounnas and D. V. Nanopoulos, Nucl. Phys. B 241, 406 (1984).

17. J. R. Ellis, C. Kounnas and D. V. Nanopoulos, Nucl. Phys. B 247 (1984) 373.

18. A. B. Lahanas and D. V. Nanopoulos, Phys. Rept. 145 (1987) 1.

19. E. Witten, Phys. Lett. B 155 (1985) 151; see also S. B. Giddings, S. Kachru and J. Polchinski, Phys. Rev. D 66, 106006 (2002) hep-th/0105097; V. Balasubramanian, P. Berglund, J. P. Conlon and F. Quevedo, JHEP 0503, 007 (2005) hepth/0502058.

20. J. Ellis, D. V. Nanopoulos and K. A. Olive, Phys. Rev. Lett. 111 (2013) 111301 arXiv:1305.1247 [hep-th]].

21. I. Antoniadis, J. R. Ellis, J. S. Hagelin and D. V. Nanopoulos, Phys. Lett. B 205 (1988) 459; Phys. Lett. B 208 (1988) 209 Addendum: [Phys. Lett. B 213 (1988) 562]; Phys. Lett. B 231 (1989) 65.

22. J. Polonyi, Budapest preprint KFKI-1977-93 (1977).

23. M. Aaboud et al. [ATLAS Collaboration], JHEP 1806, 107 (2018) arXiv:1711.01901 [hep-ex]]; M. Aaboud et al. [ATLAS Collaboration], Phys. Rev. D 97, no. 11, 112001 (2018) arXiv:1712.02332 [hep-ex]]; A. M. Sirunyan et al. [CMS Collaboration], Eur. Phys. J. C 77, no. 10, 710 (2017) arXiv:1705.04650 [hep-ex]]; A. M. Sirunyan et al. [CMS Collaboration], JHEP 1805, 025 (2018) arXiv:1802.02110 [hep-ex]].

24. L. Maiani, in Proceedings, Gif-sur-Yvette Summer School On Particle Physics, 1979, 1-52; Gerard 't Hooft and others (eds.), Recent Developments in Gauge Theories, Proceedings of the Nato Advanced Study Institute, Cargese, France, August 26 - September 8, 1979, Plenum press, New York, USA, 1980, Nato Advanced Study Institutes Series: Series B, Physics, 59.; Edward Witten, Phys. Lett. B105, 267, 1981.

25. A. H. Guth and E. J. Weinberg, Nucl. Phys. B 212, 321-364 (1983).

26. A. D. Linde, Phys. Lett. B 108, 389 (1982); A. Albrecht and P. J. Steinhardt, Phys. Rev. Lett. 48, 1220 (1982).

27. D. V. Nanopoulos, K. A. Olive, M. Srednicki and K. Tamvakis, Phys. Lett. B 123, 41 (1983);

28. S.W. Hawking, D.N. Page and C.N. Pope, Phys. Lett. B86 (1979) 175; M.J. Perry, Phys. Rev. D19 (1979) 1720; S. Giddings and A. Strominger, Nucl. Phys. B307 (1988) 854; J. Ellis and M.K. Gaillard, Phys. Lett. B88 (1979) 315; R. Barbieri, J. Ellis and M.K. Gaillard, Phys. Lett. B90 (1980) 249.

29. M. Dine, L. Randall and S. D. Thomas, Phys. Rev. Lett. 75, 398 (1995) hep$\mathrm{ph} / 9503303$.

30. G. A. Diamandis, J. R. Ellis, A. B. Lahanas and D. V. Nanopoulos, Phys. Lett. B 173, 303 (1986).

31. M. K. Gaillard, H. Murayama and K. A. Olive, Phys. Lett. B 355 (1995) 71 hep$\mathrm{ph} / 9504307$.

32. D. Volkov and V. Soroka, JETP Lett. 18, 312-314 (1973); S. Deser and B. Zumino, Phys. Lett. B 65, 369-373 (1976).

33. R. Barbieri, S. Ferrara and C. A. Savoy, Phys. Lett. B 119, 343 (1982). 
34. G. L. Kane, C. F. Kolda, L. Roszkowski and J. D. Wells, Phys. Rev. D 49 (1994) 6173 arXiv:hep-ph/9312272; V. D. Barger and C. Kao, Phys. Rev. D 57 (1998) 3131 arXiv:hep-ph/9704403; L. Roszkowski, R. Ruiz de Austri and T. Nihei, JHEP 0108 (2001) 024 arXiv:hep-ph/0106334]; A. Djouadi, M. Drees and J. L. Kneur, JHEP 0108 (2001) 055 [arXiv:hep-ph/0107316]; U. Chattopadhyay, A. Corsetti and P. Nath, Phys. Rev. D 66 (2002) 035003 arXiv:hep-ph/0201001]; J. R. Ellis, K. A. Olive and Y. Santoso, New Jour. Phys. 4 (2002) 32 arXiv:hep-ph/0202110; R. Arnowitt and B. Dutta, arXiv:hep-ph/0211417; J. R. Ellis, K. A. Olive, Y. Santoso and V. C. Spanos, Phys. Lett. B 565 (2003) 176 arXiv:hep-ph/0303043; H. Baer and C. Balazs, JCAP 0305, 006 (2003) arXiv:hep-ph/0303114; A. B. Lahanas and D. V. Nanopoulos, Phys. Lett. B 568, 55 (2003) arXiv:hep-ph/0303130; U. Chattopadhyay, A. Corsetti and P. Nath, Phys. Rev. D 68, 035005 (2003) arXiv:hep-ph/0303201; C. Munoz, Int. J. Mod. Phys. A 19, 3093 (2004) arXiv:hepph/0309346; R. Arnowitt, B. Dutta and B. Hu, arXiv:hep-ph/0310103; J. Ellis and K. A. Olive, arXiv:1001.3651 [astro-ph.CO], published in Particle dark matter, ed. G. Bertone, pp. 142-163; J. Ellis and K. A. Olive, Eur. Phys. J. C 72, 2005 (2012) arXiv:1202.3262 [hep-ph]]; O. Buchmueller et al., Eur. Phys. J. C 74 (2014) 3, 2809 arXiv:1312.5233 [hep-ph]]; O. Buchmueller, M. Citron, J. Ellis, S. Guha, J. Marrouche, K. A. Olive, K. de Vries and J. Zheng, Eur. Phys. J. C 75, no. 10, 469 (2015) Erratum: [Eur. Phys. J. C 76, no. 4, 190 (2016)] arXiv:1505.04702 [hep-ph]]; E. A. Bagnaschi et al., Eur. Phys. J. C 75, 500 (2015) arXiv:1508.01173 [hep-ph]].

35. J. Ellis, F. Luo, K. A. Olive and P. Sandick, Eur. Phys. J. C 73, no. 4, 2403 (2013) arXiv:1212.4476 [hep-ph]].

36. J. Ellis, J. L. Evans, F. Luo, N. Nagata, K. A. Olive and P. Sandick, Eur. Phys. J. C 76, no. 1, 8 (2016) arXiv:1509.08838 [hep-ph]].

37. J. Ellis, J. L. Evans, A. Mustafayev, N. Nagata and K. A. Olive, Eur. Phys. J. C 76, no. 11, 592 (2016) arXiv:1608.05370 [hep-ph]].

38. J. Ellis, J. L. Evans, F. Luo, K. A. Olive and J. Zheng, Eur. Phys. J. C 78 (2018) no.5, 425 arXiv:1801.09855[hep-ph]].

39. E. Bagnaschi et al., Eur. Phys. J. C 79, no. 2, 149 (2019) arXiv:1810.10905 [hep-ph]].

40. J. R. Ellis, K. A. Olive, Y. Santoso and V. C. Spanos, Phys. Lett. B 573 (2003) 162 arXiv:hep-ph/0305212, and Phys. Rev. D 70 (2004) 055005 arXiv:hep-ph/0405110.

41. G. D. Coughlan, W. Fischler, E. W. Kolb, S. Raby and G. G. Ross, Phys. Lett. B 131, 59 (1983); A. S. Goncharov, A. D. Linde and M. I. Vysotsky, Phys. Lett. B 147, 279 (1984); J. Ellis, D. V. Nanopoulos, and M. Quiros, Phys. Lett. B 174, 176 (1986); T. Banks, D. B. Kaplan and A. E. Nelson, Phys. Rev. D 49, 779 (1994) hep-ph/9308292; B. De Carlos, J. A. Casas, F. Quevedo, E. Roulet, Phys. Lett. B 318, 447 (1993) hep-ph/9308325.

42. T. Moroi, M. Yamaguchi and T. Yanagida Phys. Lett. B 342, 105 (1995) hepph/9409367]; M. Kawasaki, T. Moroi and T. Yanagida Phys. Lett. B 370, 52 (1996) hep-ph/9509399.

43. J. L. Evans, M. A. G. García and K. A. Olive, JCAP 1403, 022 (2014) arXiv:1311.0052 [hep-ph]].

44. S. Weinberg, Phys. Rev. Lett. 48, 1303 (1982).

45. J. R. Ellis, A. D. Linde and D. V. Nanopoulos, Phys. Lett. B 118, 59 (1982).

46. D. V. Nanopoulos, K. A. Olive and M. Srednicki, Phys. Lett. B 127, 30 (1983).

47. J. Ellis, J. Hagelin, D. Nanopoulos, K. Olive and M. Srednicki, Nucl. Phys. B 238 (1984) 453.

48. M. Y. Khlopov and A. D. Linde, Phys. Lett. B 138, 265 (1984).

49. J. R. Ellis, J. E. Kim and D. V. Nanopoulos, Phys. Lett. B 145, 181 (1984). 
50. R. Juszkiewicz, J. Silk and A. Stebbins, Phys. Lett. B 158 (1985) 463.

51. T. Moroi, H. Murayama and M. Yamaguchi, Phys. Lett. B 303, 289 (1993).

52. M. Kawasaki and T. Moroi, Prog. Theor. Phys. 93, 879 (1995) hep-ph/9403364.

53. T. Moroi, hep-ph/9503210

54. J. R. Ellis, D. V. Nanopoulos, K. A. Olive and S. J. Rey, Astropart. Phys. 4, 371 (1996) hep-ph/9505438.

55. G. F. Giudice, A. Riotto and I. Tkachev, JHEP 9911, 036 (1999) hep-ph/9911302.

56. M. Bolz, A. Brandenburg and W. Buchmuller, Nucl. Phys. B 606, 518 (2001) [Erratum-ibid. B 790, 336 (2008)] hep-ph/0012052.

57. R. H. Cyburt, J. Ellis, B. D. Fields and K. A. Olive, Phys. Rev. D 67, 103521 (2003) astro-ph/0211258.

58. K. Kohri, T. Moroi and A. Yotsuyanagi, Phys. Rev. D 73, 123511 (2006) arXiv:hep$\mathrm{ph} / 0507245$.

59. F. D. Steffen, JCAP 0609, 001 (2006) arXiv:hep-ph/0605306.

60. J. Pradler and F. D. Steffen, Phys. Rev. D 75, 023509 (2007) hep-ph/0608344.

61. J. Pradler and F. D. Steffen, Phys. Lett. B 648, 224 (2007) hep-ph/0612291.

62. M. Kawasaki, K. Kohri, T Moroi and A.Yotsuyanagi, Phys. Rev. D 78, 065011 (2008) arXiv:0804.3745 [hep-ph]].

63. V. S. Rychkov and A. Strumia, Phys. Rev. D 75, 075011 (2007) hep-ph/0701104.

64. J. Ellis, M. A. G. García, D. V. Nanopoulos, K. A. Olive and M. Peloso, JCAP 1603, no. 03, 008 (2016) arXiv:1512.05701 [astro-ph.CO]]; M. A. G. García, Y. Mambrini, K. A. Olive and M. Peloso, Phys. Rev. D 96, no. 10, 103510 (2017) arXiv:1709.01549 [hep-ph]].

65. M. A. G. García and M. A. Amin, Phys. Rev. D 98, no. 10, 103504 (2018) arXiv:1806.01865 [hep-ph]].

66. D. Lindley, Astrophys. J. 294 (1985) 1; J. R. Ellis, D. V. Nanopoulos and S. Sarkar, Nucl. Phys. B 259 (1985) 175; M. Kawasaki, K. Kohri and T. Moroi, Phys. Rev. D 63, 103502 (2001) hep-ph/0012279; M. Kawasaki, K. Kohri and T. Moroi, Phys. Rev. D 71 (2005) 083502 [arXiv:astro-ph/0408426]; J. R. Ellis, K. A. Olive and E. Vangioni, Phys. Lett. B 619, 30 (2005) arXiv:astro-ph/0503023; D. G. Cerdeno, K. Y. Choi, K. Jedamzik, L. Roszkowski and R. Ruiz de Austri, JCAP 0606, 005 (2006) arXiv:hep-ph/0509275; K. Jedamzik, K. Y. Choi, L. Roszkowski and R. Ruiz de Austri, JCAP 0607, 007 (2006) arXiv:hep-ph/0512044; S. Bailly, K. Jedamzik and G. Moultaka, Phys. Rev. D 80 (2009) 063509 arXiv:0812.0788 [hepph]]; K. Jedamzik and M. Pospelov, New J. Phys. 11, 105028 (2009) arXiv:0906.2087 [hep-ph]]; M. Pospelov and J. Pradler, Ann. Rev. Nucl. Part. Sci. 60, 539 (2010) arXiv:1011.1054 [hep-ph]];

67. R. H. Cyburt, J. Ellis, B. D. Fields, F. Luo, K. A. Olive and V. C. Spanos, JCAP 0910, 021 (2009) arXiv:0907.5003 [astro-ph.CO]]; JCAP 1010, 032 (2010) arXiv:1007.4173 [astro-ph.CO]]; JCAP 1305, 014 (2013) arXiv:1303.0574 [astroph.CO]]; R. H. Cyburt, J. R. Ellis, B. D. Fields, K. A. Olive and V. C. Spanos, JCAP 0611, 014 (2006) astro-ph/0608562 ; R. H. Cyburt, J. Ellis, B. D. Fields, F. Luo, K. A. Olive and V. C. Spanos, JCAP 1212, 037 (2012) arXiv:1209.1347 [astro-ph.CO]].

68. J. Pradler and F. D. Steffen, Phys. Lett. B 666, 181 (2008) arXiv:0710.2213 [hep$\mathrm{ph}]$.

69. J. L. Feng, A. Rajaraman and F. Takayama, Phys. Rev. D 68 (2003) 063504 arXiv:hep-ph/0306024; J. L. Feng, S. F. Su and F. Takayama, Phys. Rev. D 70 (2004) 063514 arXiv:hep-ph/0404198].

70. M. Kawasaki, K. Kohri, T. Moroi and Y. Takaesu, Phys. Rev. D 97, no. 2, 023502 
(2018) arXiv:1709.01211 [hep-ph]].

71. R. Holman, P. Ramond and G. G. Ross, Phys. Lett. B 137, 343 (1984).

72. A. D. Linde and A. Westphal, JCAP 03, 005 (2008) arXiv:0712.1610 [hep-th]].

73. M. Kawasaki, M. Yamaguchi and T. Yanagida, Phys. Rev. Lett. 85, 3572 (2000) arXiv:hep-ph/0004243.

74. R. Kallosh and A. Linde, JCAP 1011, 011 (2010) arXiv:1008.3375 [hep-th]]; R. Kallosh, A. Linde and T. Rube, Phys. Rev. D 83, 043507 (2011) arXiv:1011.5945 [hep-th]].

75. R. Kallosh, A. Linde, K. A. Olive and T. Rube, Phys. Rev. D 84, 083519 (2011) arXiv:1106.6025 [hep-th]].

76. A. S. Goncharov and A. D. Linde, Class. Quant. Grav. 1, L75 (1984).

77. C. Kounnas and M. Quiros, Phys. Lett. B 151, 189 (1985).

78. J. R. Ellis, K. Enqvist, D. V. Nanopoulos, K. A. Olive and M. Srednicki, Phys. Lett. 152B (1985) 175 Erratum: [Phys. Lett. 156B (1985) 452].

79. K. Enqvist, D. V. Nanopoulos and M. Quiros, Phys. Lett. B 159, 249 (1985); P. Binétruy and M. K. Gaillard, Phys. Rev. D 34, 3069 (1986); H. Murayama, H. Suzuki, T. Yanagida and J. Yokoyama, Phys. Rev. D 50, 2356 (1994) arXiv:hepph/9311326; S. C. Davis and M. Postma, JCAP 0803, 015 (2008) arXiv:0801.4696 [hep-ph]]; S. Antusch, M. Bastero-Gil, K. Dutta, S. F. King and P. M. Kostka, JCAP 0901, 040 (2009) arXiv:0808.2425 [hep-ph]]; S. Antusch, M. Bastero-Gil, K. Dutta, S. F. King and P. M. Kostka, Phys. Lett. B 679, 428 (2009) arXiv:0905.0905 [hepth]]; S. Antusch, K. Dutta, J. Erdmenger and S. Halter, JHEP 1104 (2011) 065 arXiv:1102.0093 [hep-th]]; R. Kallosh, A. Linde, K. A. Olive and T. Rube, Phys. Rev. D 84, 083519 (2011) arXiv:1106.6025 [hep-th]]; T. Li, Z. Li and D. V. Nanopoulos, JCAP 1402, 028 (2014) arXiv:1311.6770 [hep-ph]]; W. Buchmuller, C. Wieck and M. W. Winkler, Phys. Lett. B 736, 237 (2014) arXiv:1404.2275 [hep-th]].

80. A. R. Liddle and S. M. Leach, Phys. Rev. D 68, 103503 (2003) astro-ph/0305263.

81. J. Martin and C. Ringeval, Phys. Rev. D 82, 023511 (2010) arXiv:1004.5525 [astroph.CO]].

82. J. Ellis, D. V. Nanopoulos and K. A. Olive, JCAP 1310 (2013) 009 arXiv:1307.3537 [hep-th]].

83. G. D. Diamandis, A. B. Lahanas and K. Tamvakis, Phys. Rev. D 92 (2015) no.10, 105023 arXiv:1509.01065 [hep-th]]; G. A. Diamandis, B. C. Georgalas, K. Kaskavelis, A. B. Lahanas and G. Pavlopoulos, Phys. Rev. D 96, no. 4, 044033 (2017) arXiv:1704.07617 [hep-th]].

84. J. Ellis, D. V. Nanopoulos and K. A. Olive, Phys. Rev. D 97, no.4, 043530 (2018) arXiv:1711.11051 [hep-th]].

85. H. Buchdahl, Nuovo Cimento 23, 141 (1962); G. V. Bicknell, J. Phys. A 7, 1061 (1974); H. Buchdahl, J. Phys. A 11, 871 (1978).

86. C. Kounnas, D. Lüst and N. Toumbas, Fortsch. Phys. 63, 12 (2015) arXiv:1409.7076 [hep-th]].

87. L. Alvarez-Gaume, A. Kehagias, C. Kounnas, D. Lst and A. Riotto, Fortsch. Phys. 64, no.2-3, 176-189 (2016) arXiv:1505.07657 [hep-th]].

88. S. Kalara, N. Kaloper and K. A. Olive, Nucl. Phys. B 341, 252-272 (1990)

89. R. Kallosh, A. Linde and D. Roest, JHEP 1311, 198 (2013) arXiv:1311.0472 [hepth]].

90. R. Kallosh, A. Linde and D. Roest, JHEP 1408, 052 (2014) arXiv:1405.3646 [hepth]].

91. D. Roest and M. Scalisi, Phys. Rev. D 92, 043525 (2015) arXiv:1503.07909 [hep-th]].

92. A. Linde, JCAP 1505, 003 (2015). arXiv:1504.00663 [hep-th]]. 
93. J. Ellis, B. Nagaraj, D. V. Nanopoulos and K. A. Olive, JHEP 11, 110 (2018) arXiv:1809.10114 [hep-th]].

94. J. Ellis, D. V. Nanopoulos, K. A. Olive and S. Verner, JCAP 09, 040 (2019) arXiv:1906.10176 [hep-th]].

95. J. Ellis, B. Nagaraj, D. V. Nanopoulos, K. A. Olive and S. Verner, JHEP 10, 161 (2019) arXiv:1907.09123 [hep-th]].

96. Y. Aldabergenov, A. Chatrabhuti and S. V. Ketov, Eur. Phys. J. C 79, no.8, 713 (2019) arXiv:1907.10373 [hep-th]].

97. J. Ellis, M. A. G. García, D. V. Nanopoulos and K. A. Olive, JCAP 1507, no. 07, 050 (2015) arXiv:1505.06986 [hep-ph]];

98. J. Ellis, D. V. Nanopoulos, K. A. Olive and S. Verner, JHEP 03, 099 (2019) arXiv:1812.02192 [hep-th]].

99. S. Cecotti, Phys. Lett. B 190 (1987) 86.

100. S. Ferrara, A. Kehagias and A. Riotto, Fortsch. Phys. 62, 573 (2014) arXiv:1403.5531 [hep-th]]; S. Ferrara, A. Kehagias and A. Riotto, Fortsch. Phys. 63, 2 (2015) arXiv:1405.2353 [hep-th]]; R. Kallosh, A. Linde, B. Vercnocke and W. Chemissany, JCAP 1407, 053 (2014) arXiv:1403.7189 [hep-th]]; K. Hamaguchi, T. Moroi and T. Terada, Phys. Lett. B 733, 305 (2014) arXiv:1403.7521 [hep-ph]]; J. Ellis, M. A. G. García, D. V. Nanopoulos and K. A. Olive, JCAP 1405, 037 (2014) arXiv:1403.7518 [hep-ph]];

101. J. Ellis, M. A. G. Garcia, D. V. Nanopoulos and K. A. Olive, JCAP 08, 044 (2014) arXiv:1405.0271 [hep-ph]].

102. R. Kallosh and A. Linde, JCAP 1306 (2013) 028 arXiv:1306.3214 [hep-th]]; T. Li, Z. Li and D. V. Nanopoulos, JCAP 1404, 018 (2014) arXiv:1310.3331 [hep-ph]]; C. P. Burgess, M. Cicoli and F. Quevedo, JCAP 1311 (2013) 003 arXiv:1306.3512 [hep-th]]; F. Farakos, A. Kehagias and A. Riotto, Nucl. Phys. B 876, 187 (2013) arXiv:1307.1137 [hep-th]]; S. Ferrara, R. Kallosh, A. Linde and M. Porrati, Phys. Rev. D 88 (2013) 8, 085038 arXiv:1307.7696 [hep-th]]; W. Buchmüller, V. Domcke and C. Wieck, Phys. Lett. B 730, 155 (2014) arXiv:1309.3122 [hep-th]]; C. Pallis, JCAP 1404, 024 (2014) arXiv:1312.3623 [hep-ph]]; C. Pallis, JCAP 1408, 057 (2014) arXiv:1403.5486 [hep-ph]]; I. Antoniadis, E. Dudas, S. Ferrara and A. Sagnotti, Phys. Lett. B 733, 32 (2014) arXiv:1403.3269 [hep-th]]; T. Li, Z. Li and D. V. Nanopoulos, Eur. Phys. J. C 75, no. 2, 55 (2015) arXiv:1405.0197 [hep-th]]; W. Buchmuller, E. Dudas, L. Heurtier and C. Wieck, JHEP 1409, 053 (2014) arXiv:1407.0253 [hep-th]]; T. Terada, Y. Watanabe, Y. Yamada and J. Yokoyama, JHEP 1502, 105 (2015) arXiv:1411.6746 [hep-ph]]; W. Buchmuller, E. Dudas, L. Heurtier, A. Westphal, C. Wieck and M. W. Winkler, JHEP 1504, 058 (2015) arXiv:1501.05812 [hep-th]]; A. B. Lahanas and K. Tamvakis, Phys. Rev. D 91, no. 8, 085001 (2015) arXiv:1501.06547 [hep-th]];

103. J. Ellis, M. A. G. Garca, D. V. Nanopoulos and K. A. Olive, JCAP 01, 010 (2015) arXiv:1409.8197 [hep-ph]].

104. J. Ellis, M. A. G. García, D. V. Nanopoulos and K. A. Olive, JCAP 1510, 003 (2015) arXiv:1503.08867 [hep-ph]].

105. S. Ferrara, A. Kehagias and M. Porrati, JHEP 1508 (2015) 001 arXiv:1506.01566 [hep-th]].

106. I. Dalianis and F. Farakos, JCAP 1507, no. 07, 044 (2015) arXiv:1502.01246 [grqc]]. I. Garg and S. Mohanty, Phys. Lett. B 751, 7 (2015) arXiv:1504.07725 [hepph]]; E. Dudas and C. Wieck, JHEP 1510, 062 (2015) arXiv:1506.01253 [hep-th]]; M. Scalisi, JHEP 1512, 134 (2015) arXiv:1506.01368 [hep-th]]; arXiv:1506.01566 [hep-th]]; J. Ellis, M. A. G. García, D. V. Nanopoulos and K. A. Olive, Class. 
Quant. Grav. 33, no. 9, 094001 (2016) arXiv:1507.02308 [hep-ph]]; A. Addazi and M. Y. Khlopov, Phys. Lett. B 766, 17 (2017) arXiv:1612.06417][gr-qc]]; C. Pallis and N. Toumbas, Adv. High Energy Phys. 2017, 6759267 (2017) arXiv:1612.09202 [hepph]]; T. Kobayashi, O. Seto and T. H. Tatsuishi, arXiv:1703.09960 [hep-th]; I. Garg and S. Mohanty, Int. J. Mod. Phys. A 33, no.21, 1850127 (2018) arXiv:1711.01979 [hep-ph]]; W. Ahmed and A. Karozas, Phys. Rev. D 98, no. 2, 023538 (2018) arXiv:1804.04822 [hep-ph]]; Y. Cai, R. Deen, B. A. Ovrut and A. Purves, JHEP 1809, 001 (2018) arXiv:1804.07848 [hep-th]]; S. Khalil, A. Moursy, A. K. Saha and A. Sil, Phys. Rev. D 99, no.9, 095022 (2019) arXiv:1810.06408 [hep-ph]].

107. M. C. Romao and S. F. King, JHEP 1707, 033 (2017) arXiv:1703.08333 [hep-ph]].

108. E. Dudas, T. Gherghetta, Y. Mambrini and K. A. Olive, Phys. Rev. D 96, no. 11, 115032 (2017) arXiv:1710.07341 [hep-ph]];

109. J. Ellis, D. V. Nanopoulos, K. A. Olive and S. Verner, arXiv:2008.09099 [hep-ph].

110. J. R. Ellis, C. Kounnas and D. V. Nanopoulos, Phys. Lett. 143B, 410 (1984).

111. M. Dine, R. Kitano, A. Morisse and Y. Shirman, Phys. Rev. D 73, 123518 (2006) hepph/0604140; R. Kitano, Phys. Lett. B 641, 203 (2006) hep-ph/0607090; R. Kallosh and A. D. Linde, JHEP 0702, 002 (2007) hep-th/0611183; H. Abe, T. Higaki and T. Kobayashi, Phys. Rev. D 76 (2007) 105003 arXiv:0707.2671 [hep-th]]; J. Fan, M. Reece and L.-T. Wang, JHEP 1109, 126 (2011) [arXiv:1106.6044 [hep-ph]]; Y. Ema, R. Kitano and T. Terada, JHEP 09, 075 (2018) arXiv:1807.06940 [hepth]].

112. E. Dudas, A. Linde, Y. Mambrini, A. Mustafayev and K. A. Olive, Eur. Phys. J. C 73 (2013) 2268 arXiv:1209.0499 [hep-ph]].

113. M. A. G. García and K. A. Olive, JCAP 1309, 007 (2013) arXiv:1306.6119 [hep-ph]].

114. K. Kaneta, Y. Mambrini, K. A. Olive and S. Verner, Phys. Rev. D 101, no. 1, 015002 (2020) arXiv:1911.02463 [hep-ph]].

115. J. Ellis, D. V. Nanopoulos, K. A. Olive and S. Verner, JCAP 08, 037 (2020) arXiv:2004.00643 [hep-ph]].

116. L. J. Dixon, V. Kaplunovsky and J. Louis, Nucl. Phys. B 329, 27-82 (1990)

117. J. A. Casas, arXiv:hep-ph/9802210 [hep-ph]].

118. S. Antusch, K. Dutta, J. Erdmenger and S. Halter, JHEP 04, 065 (2011) arXiv:1102.0093 [hep-th]].

119. L. E. Ibanez and G. G. Ross, Phys. Lett. B 110, 215 (1982); K. Inoue, A. Kakuto, H. Komatsu and S. Takeshita, Prog. Theor. Phys. 68, 927 (1982) [Erratum-ibid. 70, 330 (1983)] [Prog. Theor. Phys. 70, 330 (1983)]; L. E. Ibanez, Phys. Lett. B 118, 73 (1982); J. R. Ellis, D. V. Nanopoulos and K. Tamvakis, Phys. Lett. B 121, 123 (1983); J. R. Ellis, J. S. Hagelin, D. V. Nanopoulos and K. Tamvakis, Phys. Lett. B 125, 275 (1983); L. Alvarez-Gaume, J. Polchinski and M. B. Wise, Nucl. Phys. B 221, 495 (1983).

120. J. R. Ellis, D. V. Nanopoulos and K. A. Olive, Phys. Lett. B 525, 308-314 (2002) arXiv:hep-ph/0109288 [hep-ph]].

121. S. Profumo, JHEP 0306, 052 (2003) hep-ph/0306119.

122. J. Ellis, A. Mustafayev and K. A. Olive, Eur. Phys. J. C 69, 219 (2010) arXiv:1004.5399 [hep-ph]].

123. J. Ellis, D. V. Nanopoulos and K. A. Olive, Phys. Rev. D 89 (2014) 4, 043502 arXiv:1310.4770 [hep-ph]];

124. J. Ellis, J. L. Evans, N. Nagata, D. V. Nanopoulos and K. A. Olive, Eur. Phys. J. C 77, no.4, 232 (2017) arXiv:1702.00379 [hep-ph]].

125. J. Ellis, K. Olive and L. Velasco-Sevilla, Eur. Phys. J. C 76, no.10, 562 (2016) arXiv:1605.01398 [hep-ph]]. 
126. J. L. Evans, M. Ibe, K. A. Olive and T. T. Yanagida, Eur. Phys. J. C 73, 2468 (2013) arXiv:1302.5346 [hep-ph]]; J. L. Evans, K. A. Olive, M. Ibe and T. T. Yanagida, Eur. Phys. J. C 73, 2611 (2013) arXiv:1305.7461 [hep-ph]]; J. L. Evans, M. Ibe, K. A. Olive and T. T. Yanagida, Phys. Rev. D 91, no. 5, 055008 (2015) arXiv:1412.3403 [hep-ph]]; J. L. Evans and K. A. Olive, Phys. Rev. D 90, no. 11, 115020 (2014) [arXiv:1408.5102 [hep-ph]].

127. J. L. Evans, N. Nagata and K. A. Olive, Phys. Rev. D 91, 055027 (2015) arXiv:1502.00034 [hep-ph]]; J. L. Evans, N. Nagata and K. A. Olive, Eur. Phys. J. C 79, no.6, 490 (2019) arXiv:1902.09084 [hep-ph]].

128. S. F. King and E. Perdomo, JHEP 1905, 211 (2019) arXiv:1903.08448 [hep-ph]].

129. J. Ellis, D. V. Nanopoulos, K. A. Olive and S. Verner, Phys. Rev. D 100, no. 2, 025009 (2019) arXiv:1903.05267 [hep-ph]].

130. J. Ellis, M. A. García, N. Nagata, D. V. Nanopoulos and K. A. Olive, JCAP 04, 009 (2019) arXiv:1812.08184 [hep-ph]].

131. G. Kane, K. Sinha and S. Watson, Int. J. Mod. Phys. D 24, no.08, 1530022 (2015) arXiv:1502.07746 [hep-th]].

132. I. Affleck and M. Dine, Nucl. Phys. B 249, 361-380 (1985)

133. J. R. Ellis, K. Enqvist, D. V. Nanopoulos and K. A. Olive, Phys. Lett. B 191, 343-348 (1987)

134. B. A. Campbell, M. K. Gaillard, H. Murayama and K. A. Olive, Nucl. Phys. B 538, 351-367 (1999) arXiv:hep-ph/9805300 [hep-ph]].

135. M. Endo, K. Kadota, K. A. Olive, F. Takahashi and T. T. Yanagida, JCAP 0702, 018 (2007) hep-ph/0612263.

136. H. Murayama, H. Suzuki, T. Yanagida and J.-i. Yokoyama, Phys. Rev. Lett. 70 (1993) 1912 and Phys. Rev. D 50 (1994) 2356 hep-ph/9311326]; J. R. Ellis, M. Raidal and T. Yanagida, Phys. Lett. B 581 (2004) 9 hep-ph/0303242]; D. Croon, J. Ellis and N. E. Mavromatos, Phys. Lett. B 724 (2013) 165 arXiv:1303.6253 [astro-ph.CO]]; K. Nakayama, F. Takahashi and T. T. Yanagida, Phys. Lett. B 730, 24 (2014) arXiv:1311.4253 [hep-ph]]; J. Ellis, N. E. Mavromatos and D. J. Mulryne, JCAP 1405 (2014) 012 arXiv:1401.6078 [astro-ph.CO]]; J. L. Evans, T. Gherghetta and M. Peloso, Phys. Rev. D 92, no.2, 021303 (2015) arXiv:1501.06560 [hep-ph]].

137. A. D. Dolgov and A. D. Linde, Phys. Lett. 116B, 329 (1982); L. F. Abbott, E. Farhi and M. B. Wise, Phys. Lett. 117B, 29 (1982).

138. G. F. Giudice and A. Strumia, Nucl. Phys. B 858, 63 (2012) arXiv:1108.6077 [hepph]]; E. Bagnaschi, G. F. Giudice, P. Slavich and A. Strumia, JHEP 1409, 092 (2014) arXiv:1407.4081 [hep-ph]].

139. K. Benakli, Y. Chen, E. Dudas and Y. Mambrini, Phys. Rev. D 95, no. 9, 095002 (2017) arXiv:1701.06574 [hep-ph]].

140. E. Dudas, Y. Mambrini and K. Olive, Phys. Rev. Lett. 119, no. 5, 051801 (2017) arXiv:1704.03008 [hep-ph]].

141. E. Dudas, T. Gherghetta, K. Kaneta, Y. Mambrini and K. A. Olive, Phys. Rev. D 98, no. 1, 015030 (2018) arXiv:1805.07342 [hep-ph]]; E. Dudas, T. Gherghetta, K. Kaneta, Y. Mambrini and K. A. Olive, Phys. Rev. D 100, no. 3, 035004 (2019) arXiv:1905.09243 [hep-ph]].

142. S. A. R. Ellis, T. Gherghetta, K. Kaneta and K. A. Olive, Phys. Rev. D 98, no. 5, 055009 (2018) arXiv:1807.06488 [hep-ph]].

143. K. Kaneta, Y. Mambrini and K. A. Olive, Phys. Rev. D 99, no. 6, 063508 (2019) arXiv:1901.04449 [hep-ph]].

144. J. Ellis, M. A. G. García, N. Nagata, D. V. Nanopoulos and K. A. Olive, JCAP 1611 (2016) no.11, 018 arXiv:1609.05849 [hep-ph]]. 
145. J. Ellis, M. A. G. García, N. Nagata, D. V. Nanopoulos and K. A. Olive, JCAP 1707 (2017) no.07, 006 arXiv:1704.07331 [hep-ph]].

146. J. Ellis, M. A. García, N. Nagata, D. V. Nanopoulos and K. A. Olive, Phys. Lett. B 797, 134864 (2019) arXiv:1906.08483 [hep-ph]].

147. J. Ellis, M. A. García, N. Nagata, D. V. Nanopoulos and K. A. Olive, JCAP 01, 035 (2020) arXiv:1910.11755 [hep-ph]].

148. J. Ellis, M. A. García, N. Nagata, D. V. Nanopoulos and K. A. Olive, JHEP 05, 021 (2020) arXiv:2003.03285 [hep-ph]].

149. S. Dimopoulos and H. Georgi, Nucl. Phys. B 193, 150 (1981); N. Sakai, Z. Phys. C 11, 153 (1981).

150. J. Hisano, H. Murayama and T. Yanagida, Phys. Rev. Lett. 69, 1014 (1992).

151. J. Hisano, H. Murayama and T. Yanagida, Nucl. Phys. B 402, 46 (1993) hep$\mathrm{ph} / 9207279$.

152. J. Hisano, T. Kuwahara and N. Nagata, Phys. Lett. B 723, 324 (2013) arXiv:1304.0343 [hep-ph]].

153. J. Ellis, J. L. Evans, N. Nagata, K. A. Olive and L. Velasco-Sevilla, Eur. Phys. J. C 80, no.4, 332 (2020) arXiv:1912.04888 [hep-ph]].

154. H. Georgi and D. V. Nanopoulos, Phys. Lett. B 82, 392 (1979); H. Georgi and D. V. Nanopoulos, Nucl. Phys. B 155, 52 (1979); D. V. Nanopoulos, hep-ph/0211128.

155. U. Sarkar, Phys. Lett. B 622, 118 (2005) hep-ph/0409019.

156. M. Fukugita and T. Yanagida, Phys. Lett. B 174, 45 (1986).

157. N. S. Manton, Phys. Rev. D 28, 2019 (1983); F. R. Klinkhamer and N. S. Manton, Phys. Rev. D 30, 2212 (1984); R. F. Dashen, B. Hasslacher and A. Neveu, Phys. Rev. D 10, 4130 (1974).

158. V. A. Kuzmin, V. A. Rubakov and M. E. Shaposhnikov, Phys. Lett. B 155, 36 (1985).

159. B. A. Campbell, S. Davidson and K. A. Olive, Phys. Lett. B 303, 63 (1993) hepph/9302222] B B. A. Campbell, S. Davidson and K. A. Olive, Nucl. Phys. B 399, 111 (1993) hep-ph/9302223.

160. H. Murayama and T. Yanagida, Phys. Lett. B 322, 349-354 (1994) arXiv:hepph/9310297 [hep-ph]].

161. L. Covi, E. Roulet and F. Vissani, Phys. Lett. B 384, 169 (1996). hep-ph/9605319. M. Flanz, E. A. Paschos and U. Sarkar, Phys. Lett. B 345, 248 (1995), Erratum: [Phys. Lett. B 384, 487 (1996)], Erratum: [Phys. Lett. B 382, 447 (1996)]. hep$\mathrm{ph} / 9411366$.

162. G. F. Giudice, M. Peloso, A. Riotto and I. Tkachev, JHEP 9908 (1999) 014 doi:10.1088/1126-6708/1999/08/014 hep-ph/9905242.

163. T. Asaka, K. Hamaguchi, M. Kawasaki and T. Yanagida, Phys. Lett. B 464, 12 (1999). hep-ph/9906366.

164. T. Asaka, K. Hamaguchi, M. Kawasaki and T. Yanagida, Phys. Rev. D 61, 083512 (2000). hep-ph/9907559.

165. I. Antoniadis, J. R. Ellis, J. S. Hagelin and D. V. Nanopoulos, Phys. Lett. B 194, 231 (1987).

166. J. R. Ellis, D. V. Nanopoulos and K. A. Olive, Phys. Lett. B 300, 121 (1993) hepph/9211325.

167. S. M. Barr, Phys. Lett. 112B (1982) 219; S. M. Barr, Phys. Rev. D 40, 2457 (1989).

168. J. P. Derendinger, J. E. Kim and D. V. Nanopoulos, Phys. Lett. 139B (1984) 170.

169. K. Hamaguchi, S. Hor and N. Nagata, arXiv:2008.08940 [hep-ph]].

170. D. V. Nanopoulos and K. Tamvakis, Phys. Lett. B 110, 449 (1982); M. Srednicki, Nucl. Phys. B 202, 327 (1982); M. Srednicki, Nucl. Phys. B 206, 132 (1982); D. V. Nanopoulos, K. A. Olive, M. Srednicki and K. Tamvakis, Phys. Lett. 124B, 
$171(1983)$.

171. D. V. Nanopoulos, K. A. Olive and K. Tamvakis, Phys. Lett. B 115, 15 (1982).

172. B. A. Campbell, J. R. Ellis, J. S. Hagelin, D. V. Nanopoulos and K. A. Olive, Phys. Lett. B 197, 355 (1987).

173. J. R. Ellis, J. L. Lopez, D. V. Nanopoulos and K. A. Olive, Phys. Lett. B 308, 70 (1993) hep-ph/9303307.

174. P. Minkowski, Phys. Lett. B 67, 421 (1977); T. Yanagida, Conf. Proc. C 7902131, 95 (1979); M. Gell-Mann, P. Ramond and R. Slansky, Conf. Proc. C 790927, 315 (1979) arXiv:1306.4669 [hep-th]]; S. L. Glashow, NATO Sci. Ser. B 59, 687 (1980); R. N. Mohapatra and G. Senjanovic, Phys. Rev. Lett. 44, 912 (1980); R. N. Mohapatra and G. Senjanovic, Phys. Rev. D 23, 165 (1981); J. Schechter and J. W. F. Valle, Phys. Rev. D 22, 2227 (1980); J. Schechter and J. W. F. Valle, Phys. Rev. D 25, 774 (1982).

175. H. Georgi and D. V. Nanopoulos, Nucl. Phys. B 155, 52 (1979).

176. NuFIT Collaboration, http://www.nu-fit.org/ I. Esteban, M. C. Gonzalez-García, A. Hernandez-Cabezudo, M. Maltoni and T. Schwetz, arXiv:1811.05487 [hep-ph]; F. Capozzi, E. Di Valentino, E. Lisi, A. Marrone, A. Melchiorri and A. Palazzo, Phys. Rev. D 95, no. 9, 096014 (2017) arXiv:1703.04471 [hep-ph]].

177. L. Covi, E. Roulet and F. Vissani, Phys. Lett. B 384, 169 (1996) hep-ph/9605319.

178. K. A. Olive and M. Srednicki, Nucl. Phys. B 355, 208 (1991).

179. J. Ellis and K. A. Olive, in Particle dark matter, ed. G. Bertone, pp142-163 arXiv:1001.3651 [astro-ph.CO]].

180. K. A. Olive, D. N. Schramm and G. Steigman, Nucl. Phys. B 180, 497 (1981).

181. K. Griest and D. Seckel, Phys. Rev. D 43, 3191 (1991).

182. C. Boehm, A. Djouadi and M. Drees, Phys. Rev. D 62, 035012 (2000) arXiv:hepph/9911496; J. R. Ellis, K. A. Olive and Y. Santoso, Astropart. Phys. 18, 395 (2003) arXiv:hep-ph/0112113 ; J. L. Diaz-Cruz, J. R. Ellis, K. A. Olive and Y. Santoso, JHEP 0705, 003 (2007) arXiv:hep-ph/0701229; I. Gogoladze, S. Raza and Q. Shafi, Phys. Lett. B 706, 345 (2012) [arXiv:1104.3566 [hep-ph]]; M. A. Ajaib, T. Li and Q. Shafi, Phys. Rev. D 85, 055021 (2012) arXiv:1111.4467 [hep-ph]]; J. Harz, B. Herrmann, M. Klasen, K. Kovarik and Q. L. Boulc'h, Phys. Rev. D 87 (2013) 5, 054031 arXiv:1212.5241; J. Harz, B. Herrmann, M. Klasen and K. Kovarik, Phys. Rev. D 91 (2015) 3, 034028 arXiv:1409.2898 [hep-ph]]; A. Ibarra, A. Pierce, N. R. Shah and S. Vogl, Phys. Rev. D 91, no. 9, 095018 (2015) arXiv:1501.03164 [hep-ph]].

183. J. Edsjö, M. Schelke, P. Ullio and P. Gondolo, JCAP 0304, 001 (2003) hep$\mathrm{ph} / 0301106$.

184. J. Ellis, K. A. Olive and J. Zheng, Eur. Phys. J. C 74 (2014) 2947 arXiv:1404.5571 [hep-ph]].

185. O. Buchmueller, M. Citron, J. Ellis, S. Guha, J. Marrouche, K. A. Olive, K. de Vries and J. Zheng, Eur. Phys. J. C 75, no. 10, 469 (2015) arXiv:1505.04702 [hep-ph]].

186. S. Raza, Q. Shafi and C. S. Ün, Phys. Rev. D 92, no. 5, 055010 (2015) arXiv:1412.7672 [hep-ph]].

187. R. Arnowitt, B. Dutta and Y. Santoso, Nucl. Phys. B 606 (2001) 59 arXiv:hepph/0102181; M. E. Gómez, G. Lazarides and C. Pallis, Phys. Rev. D D61 (2000) 123512 arXiv:hep-ph/9907261] Phys. Lett. B487 (2000) 313 arXiv:hepph/0004028; ; Nucl. Phys. B B638 (2002) 165 T. Nihei, L. Roszkowski and R. Ruiz de Austri, JHEP 0207 (2002) 024 arXiv:hep-ph/0206266.

188. M. Citron, J. Ellis, F. Luo, J. Marrouche, K. A. Olive and K. J. de Vries, Phys. Rev. D 87, no. 3, 036012 (2013) arXiv:1212.2886 [hep-ph]], and references therein.

189. N. Desai, J. Ellis, F. Luo and J. Marrouche, Phys. Rev. D 90, no. 5, 055031 (2014) 
arXiv:1404.5061 [hep-ph]].

190. M. Drees and M. M. Nojiri, Phys. Rev. D 47 (1993) 376 arXiv:hep-ph/9207234;

H. Baer and M. Brhlik, Phys. Rev. D 53 (1996) 597 arXiv:hep-ph/9508321]; Phys. Rev. D 57 (1998) 567 arXiv:hep-ph/9706509; H. Baer, M. Brhlik, M. A. Diaz, J. Ferrandis, P. Mercadante, P. Quintana and X. Tata, Phys. Rev. D 63 (2001) 015007 arXiv:hep-ph/0005027; J. R. Ellis, T. Falk, G. Ganis, K. A. Olive and M. Srednicki, Phys. Lett. B 510 (2001) 236 arXiv:hep-ph/0102098.

191. J. L. Feng, K. T. Matchev and T. Moroi, Phys. Rev. Lett. 84, 2322 (2000) arXiv:hepph/9908309; ; H. Baer, T. Krupovnickas, S. Profumo and P. Ullio, JHEP 0510 (2005) 020 hep-ph/0507282] ;. L. Feng, K. T. Matchev and D. Sanford, Phys. Rev. D 85, 075007 (2012) arXiv:1112.3021] [hep-ph]]; P. Draper, J. Feng, P. Kant, S. Profumo and D. Sanford, Phys. Rev. D 88, 015025 (2013) arXiv:1304.1159 [hep-ph]].

192. L. Calibbi, Y. Mambrini and S. K. Vempati, JHEP 0709, 081 (2007) $\operatorname{arXiv:0704.3518}$ [hep-ph]]; L. Calibbi, A. Faccia, A. Masiero and S. K. Vempati, Phys. Rev. D 74, 116002 (2006) arXiv:hep-ph/0605139; E. Carquin, J. Ellis, M. E. Gomez, S. Lola and J. Rodriguez-Quintero, JHEP 0905 (2009) 026 arXiv:0812.4243 [hep-ph]].

193. J. Ellis, A. Mustafayev and K. A. Olive, Eur. Phys. J. C 69, 201 (2010) arXiv:1003.3677 [hep-ph]].

194. J. Ellis, A. Mustafayev and K. A. Olive, Eur. Phys. J. C 71, 1689 (2011) arXiv:1103.5140 [hep-ph]].

195. H. Bahl, T. Hahn, S. Heinemeyer, W. Hollik, S. Passehr, H. Rzehak and G. Weiglein, arXiv:1811.09073 [hep-ph].

196. S. Dimopoulos, S. Raby and F. Wilczek, Phys. Lett. 112B (1982) 133; J. R. Ellis, D. V. Nanopoulos and S. Rudaz, Nucl. Phys. B 202 (1982) 43.

197. T. Goto and T. Nihei, Phys. Rev. D 59, 115009 (1999) hep-ph/9808255].

198. H. Murayama and A. Pierce, Phys. Rev. D 65, 055009 (2002) hep-ph/0108104].

199. J. Hisano, D. Kobayashi, T. Kuwahara and N. Nagata, JHEP 1307, 038 (2013) arXiv:1304.3651 [hep-ph]]; N. Nagata and S. Shirai, JHEP 1403, 049 (2014) arXiv:1312.7854 [hep-ph]]; N. Nagata, Ph.D. Thesis, http://doi.org/10.15083/ 00006623

200. K. Abe et al. [Super-Kamiokande Collaboration], Phys. Rev. D 90, no. 7, 072005 (2014) arXiv:1408.1195 [hep-ex]]; V. Takhistov [Super-Kamiokande Collaboration], arXiv:1605.03235 [hep-ex].

201. F. An et al. [JUNO Collaboration], J. Phys. G 43 (2016) no.3, 030401 arXiv:1507.05613 [physics.ins-det]].

202. R. Acciarri et al. [DUNE Collaboration], arXiv:1512.06148 [physics.ins-det]; B. Abi et al. [DUNE Collaboration], arXiv:1807.10334 [physics.ins-det].

203. K. Abe et al. [Hyper-Kamiokande Collaboration], arXiv:1805.04163 [physics.ins-det]. 